\title{
The effect of a water molecule on the mechanism of formation of Compound 0 in HRP
}

Etienne Derat, ${ }^{a}$ Sason Shaik, ${ }^{a^{*}}$ Carme Rovira, ${ }^{\mathrm{b}}$ Pietro Vidossich, ${ }^{\mathrm{b}}$ Mercedes Alfonso-Prieto ${ }^{\mathrm{b}}$

a) Department of Organic Chemistry and the Lise Meitner-Minerva Center for Computational Quantum Chemistry, Hebrew University of Jerusalem, Givat Ram Campus, 91904 Jerusalem, Israël, Tel +972 (0)2 6585909, Fax +972 (0)2 6584033 b) Centre especial de Recerca en Química Teòrica, Parc Científic de Barcelona, Josep Samitier 1-5, 08028 Barcelona, Spain.

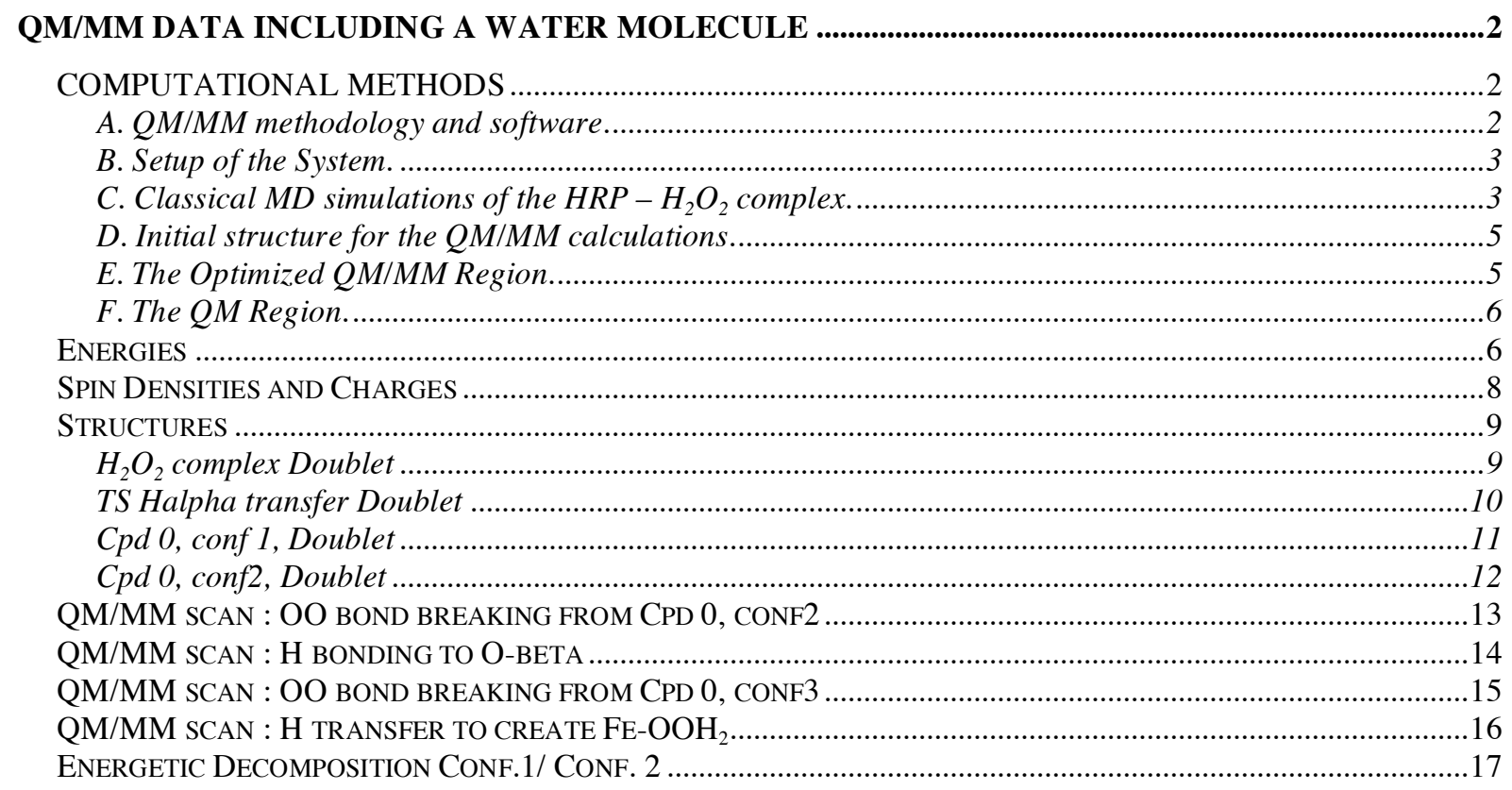

QM/MM DATA WITHOUT A WATER MOLECULE .................................................................................................19

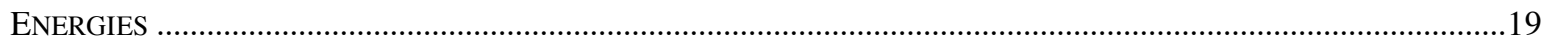

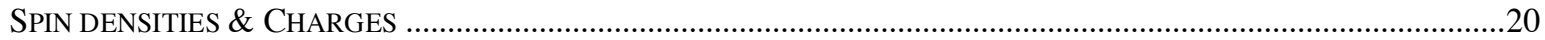

STRUCTURES ........................................21

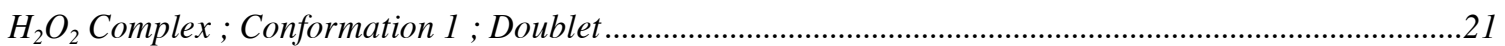

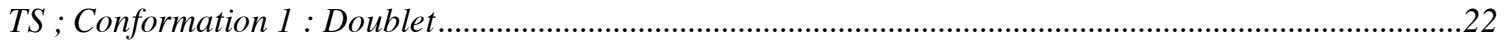

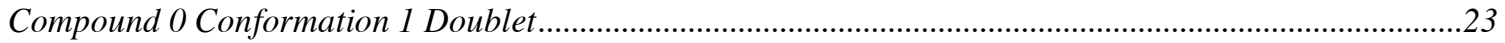

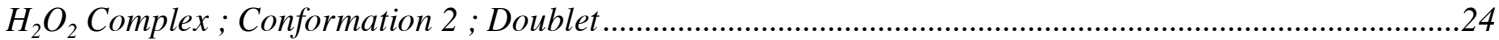

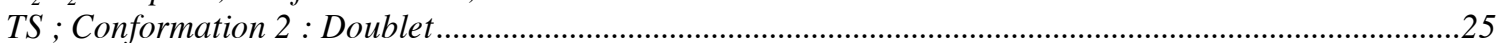

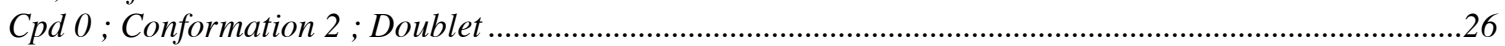

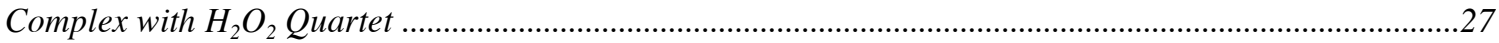

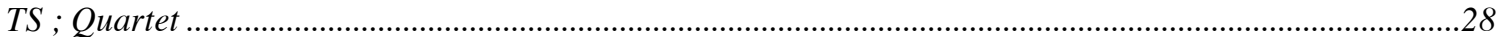

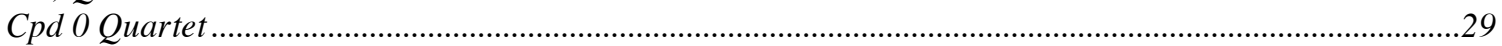

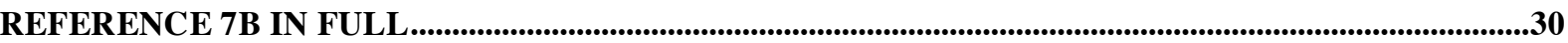

CPMD AND QM/MM CALCULATIONS ON THE CONVERSION OF CONF 2 TO CONF 3 ................31

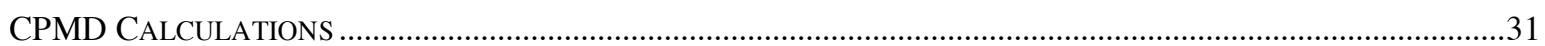

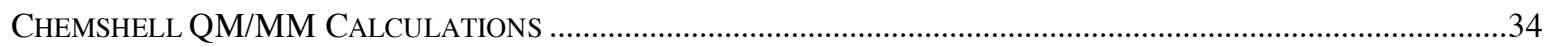

CLASSICAL SIMULATIONS ON WATER ESCAPE FROM THE BINDING POCKET ........................35 


\section{QM/MM Data including a water molecule}

\section{COMPUTATIONAL METHODS}

\section{A. QM/MM methodology and software.}

The QM/MM method ${ }^{1}$ divides the total enzyme into two subsystems, the active center and the remaining parts, comprising the protein, ions, crystal water and surface water. The active center is treated by a quantum chemical (QM) method, which in the present case is the unrestricted UB3LYP ${ }^{2}$ functional of density functional theory (DFT) and for some single points the UPBE0 $0^{3}$ functional. The remaining parts are all treated by molecular mechanics (MM), using a protein force field. The two subsystems are allowed to interact by electrostatic and van der Waals type terms, such that after optimization, the QM subsystem adapts its geometry, electronic structure, and charge distribution to the polarity and steric constraints of the protein environment, and to its hydrogen bonding machinery.

Since the QM subsystem is extracted from the protein, the dangling bonds at the boundary have to be handled specifically. In the present study we use the electrostatic embedding method that incorporates the MM charges into the one electronic Hamiltonian of the QM procedure, while the dangling bonds are capped with $\mathrm{H}$-atoms (the link atom method) and the effect of this replacement is corrected by the charge shift method. ${ }^{4}$ The QM/MM calculations are performed with the ChemShell software ${ }^{5}$ that integrates the TURBOMOLE package for $\mathrm{QM},{ }^{6}$ and the DL-POLY program, ${ }^{7}$ which uses the CHARMM22 force field, ${ }^{8}$ for MM.

Two basis sets were used in the study, B1 and B2: B1 consists of an effective core potential on iron coupled with the double $\zeta$ basis set; LACVP, ${ }^{9}$ and $6-31 \mathrm{G}$ on all other atoms. B2 describes the iron by the Wachters all electron basis $\operatorname{set}^{10}$ augmented with diffuse $\mathrm{d}$ and $\mathrm{f}$

${ }^{1}$ a) Aqvist, J.; Warshel, A. Chem. Rev. 1993, 93, 2523-2544. b) Gao, J. in Reviews in Computational Chemistry; 1995, Lipkowitz, K. B., Boyd, D. B., Eds.; VCH: Weinheim, Vol. 7, p 119.

2 a) Becke, A. D. Phys. Rev. A 1988, 36, 3098-3100. b) Lee, C.; Yang, W.; Parr, R. G. Phys. Rev. B 1988, 37, 785-789. c) Becke, A. D. J. Chem. Phys. 1993, 98, 5648-5652.

${ }^{3}$ a) Perdew, J. P.; Burke, K.; Ernzerhof, M. Phys. Rev. Lett. 1996, 77, 3865-3868. b) Perdew, J. P.; Ernzerhof, M.; Burke, K. J. Chem. Phys., 1996, 105, 9982-9985.

${ }^{4}$ Bakowies, D., Thiel, W. J. Phys. Chem. 1996, 100, 10580-10594

${ }^{5}$ Sherwood P.; de Vries A.H.; Guest M.F.; Schreckenbach G.; Catlow C.R.A.; French S.A.; Sokol A.A.; Bromley S.T.; Thiel W.; Turner A.J.; Billeter S.; Terstegen F.; Thiel S.; Kendrick J.; Rogers S.C.; Casci J.; Watson M.; King F.; Karlsen E.; Sjovoll M.; Fahmi A.; Schafer A.; Lennartz C. J. Mol. Struc. (Theochem) 2003, 632, 1-28

${ }^{6}$ Ahlrichs, R.; Bär, M.; Häser, M.; Horn, H.; Kölmel, C. Chem. Phys. Letters 1989, 162, 165169.

${ }^{7}$ Smith, W.; Forester, T. J. Mol. Graph. 1996, 14, 136-141.

${ }^{8}$ MacKerell, A. D., Jr. et al. J. Phys. Chem. B 1998, 102, 3586-3616.

${ }^{9}$ LACVP is derived from LANL2DZ: Hay, J. P.; Wadt, W. R. J. Chem. Phys. 1985, 82, 299310.

${ }^{10}$ Wachters, A. J. H. J. Chem. Phys. 1970, 52, 1033-1036. 
polarization functions; the first coordination sphere of iron and other electronegative atoms are represented by $6-31+\mathrm{G}(\mathrm{d})$, while the remaining atoms by $6-31 \mathrm{G}(\mathrm{d})$.

\section{B. Setup of the System.}

We started from the experimental X-ray structure of HRP Cpd I, ${ }^{11}$ reported by Berglund et al. (PDB code: $1 \mathrm{HCH}$ ). A complete model of the solvated enzyme was built by modifying the active site to include $\mathrm{H}_{2} \mathrm{O}_{2}$ and by adding the missing hydrogen atoms and a $16 \AA$-thick water solvent layer. The complete system consisted of 19452 atoms, including 13395 atoms in the solvent.

The total charge of the so generated system was zero, and corresponded to the following protonated state of the various residues: Aspartates (Asp) and glutamates (Glu) are negatively charged (Asp 8, 20, 29, 43, 50, 56, 66, 81, 99, 125, 132, 150, 162, 182, 194, 220, 222, 230, 247, 258, 282; Glu 25, 64, 88, 238, 239, 249, 279), and arginines (Arg) and lysines (Lys) are positively charged (Arg 19, 27, 31, 38, 62, 75, 82, 93, 118, 123, 124, 153, 159, 178, 183, 206, $224,264,283,298,302$; Lys $65,84,149,174,232,241)$. One more positive charge is "located" on the heme moiety. The histidines (His40, His42, His170) were singly protonated, and hence electrically neutral. Of these His residues, one is the proximal ligand His170, the other is the distal residue His42 that was kept neutral to represent the situation in neutral to basic $p \mathrm{H}$.

\section{Classical MD simulations of the $\mathrm{HRP}-\mathrm{H}_{2} \mathrm{O}_{2}$ complex.}

Classical Molecular Dynamics (MD) simulations were performed to explore water dynamics within the enzyme active site. The calculations were based on the $\mathrm{HRP}-\mathrm{H}_{2} \mathrm{O}_{2}$ complex previously reported (E. Derat \& S. Shaik, J. Phys. Chem. B, 2006, 110, 10526-10533). The complex was solvated with 12033 water molecules and $2 \mathrm{Cl}^{-}$ions were included in the model to neutralize the simulation cell. The AMBER (version 99) (W.C. Cornell et al., J. Am. Chem. Soc. 1995 , 117, 5179-5197; T.E. Cheatham et al., J. Biomol. Struct. Dyn.1999, 16, 845-862) and TIP3P (W.L. Jorgensen et al., J. Chem. Phys. 1983, 79, 926-935) force fields were used for the protein and for water, respectively. GAFF parameters (J.M. Wang et al., J. Comput. Chem. 2004, 25, 1157-1174) were used for hydrogen peroxide. The force field parameters for the heme moiety were adapted from those of the AMBER distribution, which were developed for a Fe(II) porphyrin, adding a +1 charge on the Fe. Calculations were performed with the NAMD program (Kale et al., J. Comp. Phys., 1999, 151, 283-312). Periodic boundary conditions were applied. The size of the simulation cells was 73 × 81 × $69 \AA$ A. Long range electrostatic interactions were treated with the PME method. A $8 \AA$ cutoff for the real part of the electrostatic and for van der Waals interactions was used. The integration timestep was 2 fs. We performed $0.5 \mathrm{~ns}$ of simulation at constant temperature $(300 \mathrm{~K})$ and pressure $(1 \mathrm{~atm})$ keeping the protein and $\mathrm{H}_{2} \mathrm{O}_{2}$ atoms frozen at the initial the conformation (simulation md1). In a subsequent step, $\mathrm{H}_{2} \mathrm{O}_{2}$ was relaxed ( $1 \mathrm{~ns}$, simulation md2).

Here we report on the water dynamics in the distal pocket. The average number of water molecules in the distal pocket within $5 \AA$ from $\mathrm{H}_{2} \mathrm{O}_{2}$ is $1.1 \pm 0.4$ in $\mathrm{md} 1$ and $2.7 \pm 0.8$ in md2 (Fig. 1). The number of water molecules close to $\mathrm{H}_{2} \mathrm{O}_{2}$ increases in md2 because of the change of conformation of $\mathrm{H}_{2} \mathrm{O}_{2}$, which is brought more towards the water channel (Fig. 2). This conformation is maintained during the simulation and is similar to the one reported in a similar study (Filizola and Loew, J. Am. Chem. Soc., 2000, 122, 18-25). Water molecules in the distal

${ }^{11}$ Berglund, G. I.; Carlsson, G. H.; Smith, A. T.; Szoeke, H.; Henriksen, A.; Hajdu, J. Nature 2002, 417, 463-468. 
pocket readily exchange with those in the channel. During md1, 4 different molecules were observed within $5 \AA$ from hydrogen peroxide, 12 during md2. Some conformations along the simulation md2 display a water molecule bridging $\mathrm{H}_{2} \mathrm{O}_{2}$ and the distal His (Fig. 3).

It should also be pointed out that a $1.5 \mathrm{~ns}$ simulation is not enough to quantify the occupancy/probability of a single water molecule in the binding pocket, nor it is the goal of the present study to firmly establish the occupancy of a given water molecule. Nevertheless, it is clear from our results that a single water molecule easily enters the binding pocket. As shown in the paper, this water is responsible of a dramatic lowering of the reaction energy barrier reduction. Altogether, this provides a plausible explanation for the manner in which theory may be brought in line with the observed reaction rates.

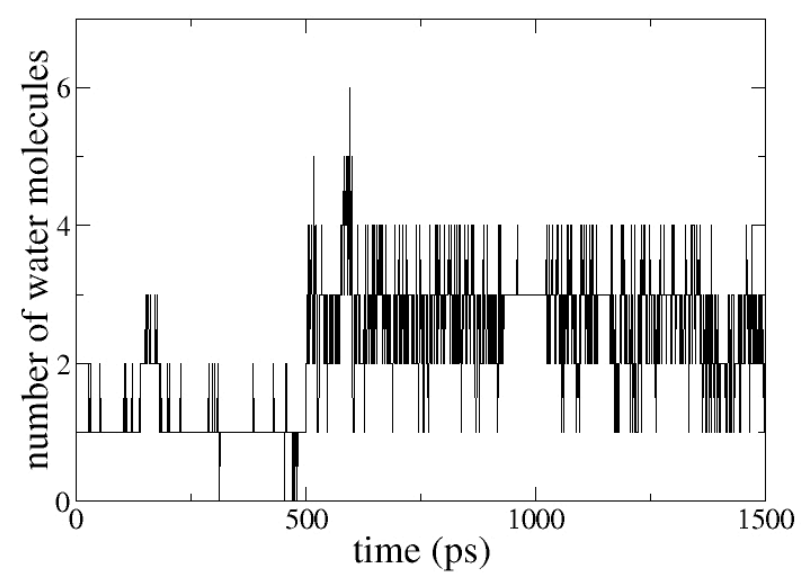

Figure S1. Number of water molecules within $5 \AA$ from hydrogen peroxide.
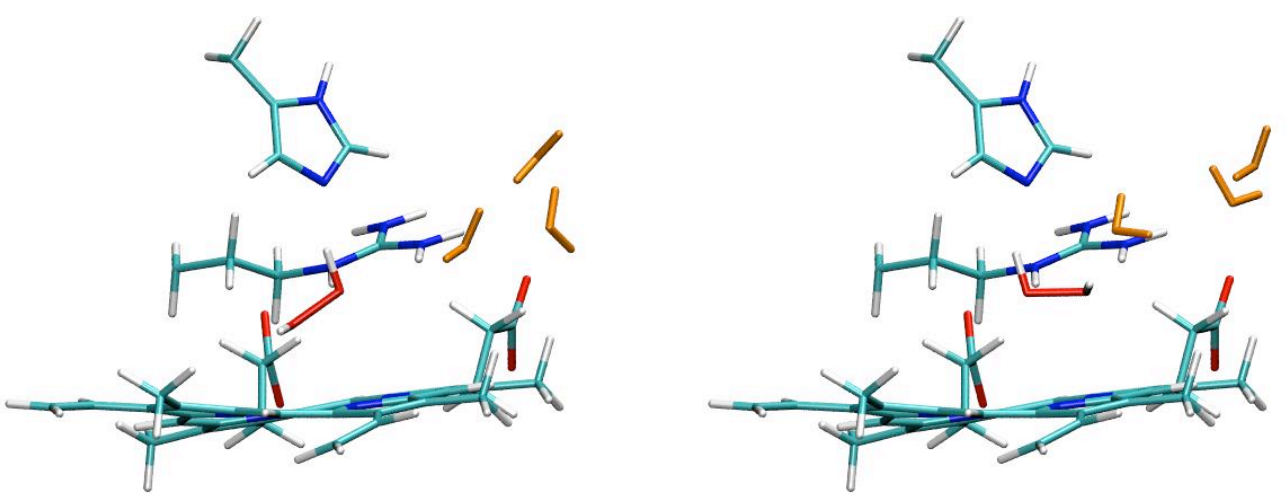

Figure S2. Snapshots of the HRP active site during simulations md1 (left) and md2 (right).

Distal pocket water molecules are colored in orange. 


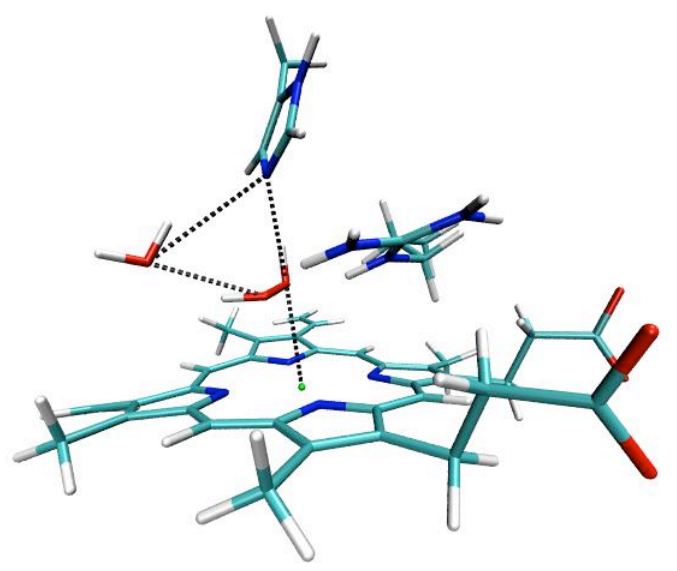

Figure S3. Perspective view of a snapshot of the HRP active site during simulation md2.

\section{Initial structure for the QM/MM calculations.}

We started the QM/MM calculations from the snapshot obtained from classical MD displayed in Figure S3. The model of the solvated enzyme was rebuilt by adding $16 \AA$-thick water solvent layer instead of a periodic box, since our QM/MM scheme can not use periodic boundary conditions. The complete system consisted of 19452 atoms, including 13395 atoms in the solvent.

The total charge of the so generated system was zero, and corresponded to the following protonated state of the various residues: Aspartates (Asp) and glutamates (Glu) are negatively charged (Asp 8, 20, 29, 43, 50, 56, 66, 81, 99, 125, 132, 150, 162, 182, 194, 220, 222, 230, 247, 258, 282; Glu 25, 64, 88, 238, 239, 249, 279), and arginines (Arg) and lysines (Lys) are positively charged (Arg 19, 27, 31, 38, 62, 75, 82, 93, 118, 123, 124, 153, 159, 178, 183, 206, $224,264,283,298,302$; Lys $65,84,149,174,232,241)$. One more positive charge is "located" on the heme moiety. The histidines (His40, His42, His170) were singly protonated, and hence electrically neutral. Of these His residues, one is the proximal ligand His170, the other is the distal residue His42 that was kept neutral to represent the situation in neutral to basic $p \mathrm{H}$.

\section{E. The Optimized QM/MM Region.}

In addition to the QM region (see below) the QM/MM geometry optimization included the following residues: $\operatorname{Arg}_{31}, \mathrm{Ser}_{35}, \mathrm{Arg}_{38}, \mathrm{Phe}_{41}, \mathrm{His}_{42}, \mathrm{Ser}_{73}, \mathrm{Leu}_{138}, \mathrm{Pro}_{139}, \mathrm{Ala}_{140}, \mathrm{Pro}_{141}$, $\mathrm{Ser}_{167}, \mathrm{His}_{170}, \mathrm{Gln}_{176}, \mathrm{Ile}_{244}, \mathrm{Asp}_{247}, \mathrm{Phe}_{221}, \mathrm{Tyr}_{233}, \mathrm{~W}_{33}, \mathrm{~W}_{133}, \mathrm{~W}_{278}, \mathrm{~W}_{332}, \mathrm{~W}_{355}, \mathrm{~W}_{423}$, and two solvent water: W2402 and W2407, this latter being the one included in the QM region. This water molecule originates in the water layer in the surface and when it enters the pocket it is located near Pro139. Thus, whereas in our previous study (E. Derat \& S. Shaik, J. Phys. Chem. B, 2006, 110, 10526-10533), one molecule of water (W420) was formed only after the formation of Compound I, here there is a water molecule from the start of the process. 


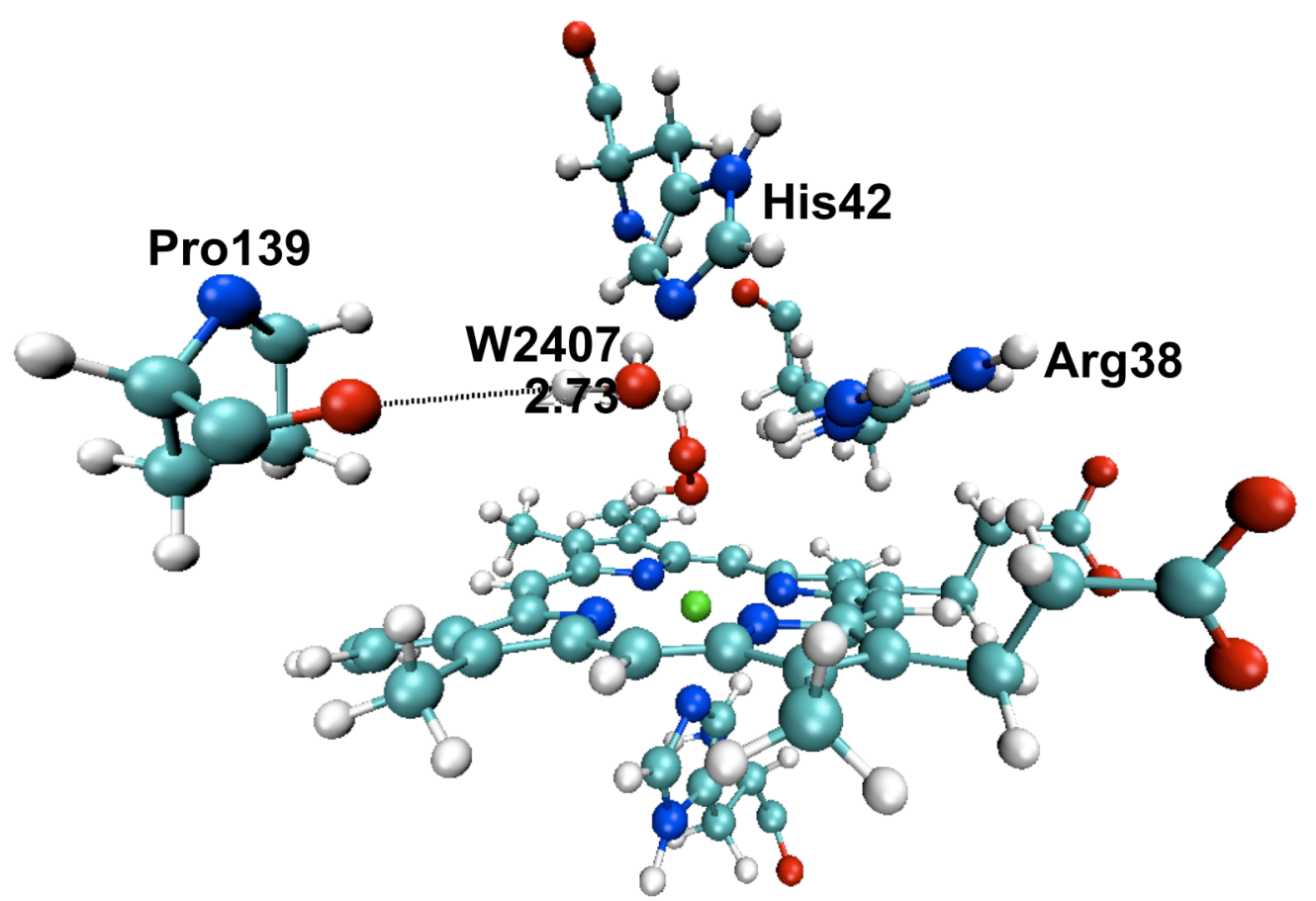

Figure S4. View of the active site of HRP in complex with its susbstrate $\mathrm{H}_{2} \mathrm{O}_{2}$, showing the hydrogen bond between Pro139 and the solvent water molecule (W2407).

\section{F. The QM Region.}

Based on previous study of $\mathrm{Cpd} \mathrm{I}^{12}$ using the same methodology, the mechanism was investigated using a QM subsystem comprising of the heme with its distal ligand (e.g., $\mathrm{H}_{2} \mathrm{O}_{2}$ ), Arg38, His42 and W2407.

\section{Energies}

Table S1 : Absolute QM and QM/MM energies at the B3LYP/B1 and B3LYP/B2//B3LYP/B1 levels.

\begin{tabular}{|l|r|r|r|r|}
\hline & & B3LYP/B1 & B3LYP/B2/B3LYP/B1 \\
\hline Structures & $\mathrm{E}(\mathrm{QM})$ & $\mathrm{E}(\mathrm{QM} / \mathrm{MM})$ & $\mathrm{E}(\mathrm{QM})$ & $\mathrm{E}(\mathrm{QM} / \mathrm{MM})$ \\
\hline $\mathrm{H}_{2} \mathrm{O}_{2}$ complex Doublet & -1997.868964 & -2079.3384 & -3138.636128 & -3220.105514 \\
\hline TS Halpha transfer Doublet & -1997.870699 & -2079.33627 & -3138.630843 & -3220.096402 \\
\hline Cpd 0, conf 1, Doublet & -1997.872042 & -2079.34288 & -3138.629415 & -3220.100201 \\
\hline Compound0, conf 2, Doublet & -1997.897031 & -2079.368012 & -3138.653591 & -3220.124541 \\
\hline
\end{tabular}

Table S2 : Relative QM and QM/MM energies at the B3LYP/B1 and B3LYP/B2//B3LYP/B1 levels.

\begin{tabular}{|l|r|r|r|r|}
\hline & \multicolumn{3}{|c|}{$\mathrm{B} 3 \mathrm{LYP} / \mathrm{B} 1$} & $\mathrm{~B} 3 \mathrm{LYP} / \mathrm{B} 2 / / \mathrm{B} 3 \mathrm{LYP} / \mathrm{B} 1$ \\
\hline Structures & $\Delta \mathrm{E}(\mathrm{QM})$ & $\Delta \mathrm{E}(\mathrm{QM} / \mathrm{MM})$ & $\Delta \mathrm{E}(\mathrm{QM})$ & $\Delta \mathrm{E}(\mathrm{QM} / \mathrm{MM})$ \\
\hline $\mathrm{H}_{2} \mathrm{O}_{2}$ complex Doublet & 0.00 & 0.00 & 0.00 & 0.00 \\
\hline TS Halpha transfer Doublet & -1.09 & 1.34 & 3.32 & 5.72 \\
\hline
\end{tabular}

${ }^{12}$ Derat, E.; Cohen, S.; Shaik, S.; Altun, A.; Thiel, W. J. Am. Chem. Soc. 2005, 127, 1361113621. 


\begin{tabular}{|l|r|r|r|r|}
\hline Cpd 0, conf 1, Doublet & -1.93 & -2.81 & 4.21 & 3.33 \\
\hline Cpd 0, conf 2, Doublet & -17.61 & -18.58 & -10.96 & -11.94 \\
\hline
\end{tabular}

Table S3 : Absolute and Relative QM and QM/MM energies at the PBE0/B2//B3LYP/B1 levels.

\begin{tabular}{|l|r|r|r|r|}
\hline Structures & $\mathrm{E}(\mathrm{QM})$ & $\mathrm{E}(\mathrm{QM} / \mathrm{MM})$ & $\Delta \mathrm{E}(\mathrm{QM})$ & $\Delta \mathrm{E}(\mathrm{QM} / \mathrm{MM})$ \\
\hline $\mathrm{H}_{2} \mathrm{O}_{2}$ complex Doublet & -3136.2744 & -3217.7438 & 0.00 & 0.00 \\
\hline TS H-alpha transfer Doublet- -3136.2698 & -3217.7354 & 2.89 & 5.29 \\
\hline Cpd 0, conf 1, Doublet & -3136.2672 & -3217.738 & 4.54 & 3.67 \\
\hline Cpd 0, conf 2, Doublet & -3136.2926 & -3217.7636 & -11.42 & -12.40 \\
\hline
\end{tabular}




\section{Spin Densities and Charges}

Table S4 : Spin Densities and Charges at the B3LYP/B1 level

\begin{tabular}{|l|l|l|l|l|l|r|r|}
\hline Spin densities & $\mathrm{Fe}$ & $\mathrm{H}_{2} \mathrm{O}_{2}$ & Porp & ImH & Arg38 & His42 & $\mathrm{H}_{2} \mathrm{O}$ \\
\hline $\mathrm{H}_{2} \mathrm{O}_{2}$ complex Doublet & 1.0588 & -0.0065 & -0.0407 & -0.0119 & 0.0004 & 0 & -0.0001 \\
\hline TS H-alpha transfer Doublet & 1.0589 & -0.0062 & -0.0418 & -0.0115 & 0.0005 & 0 & 0.0001 \\
\hline Cpd 0, conf 1, Doublet & 1.059 & -0.0051 & -0.0425 & -0.0118 & 0.0003 & 0.0001 & 0 \\
\hline Cpd 0, conf 2, Doublet & 1.0401 & 0.0127 & -0.0502 & -0.0047 & 0.0017 & 0 & 0.0004 \\
\hline Charges & $\mathrm{Fe}$ & $\mathrm{H} 2 \mathrm{O} 2$ & Porp & $\mathrm{ImH}$ & Arg38 & His42 & H2O \\
\hline $\mathrm{H}_{2} \mathrm{O}_{2}$ complex Doublet & 0.7528 & 0.0233 & -0.3041 & 0.4031 & 0.8616 & 0.159 & 0.1043 \\
\hline TS H-alpha transfer Doublet & 0.7635 & -0.0006 & -0.3388 & 0.3917 & 0.8503 & 0.2084 & 0.1255 \\
\hline Cpd 0, conf 1, Doublet & 0.75 & -0.0283 & -0.3693 & 0.3768 & 0.8033 & 0.3895 & 0.078 \\
\hline Cpd 0, conf 2, Doublet & 0.7099 & -0.0089 & -0.3688 & 0.3733 & 0.8307 & 0.388 & 0.0758 \\
\hline
\end{tabular}

Table S5 : Spin Densities and Charges at the B3LYP/B2 level

\begin{tabular}{|l|l|l|l|l|r|r|r|}
\hline Spin densities & $\mathrm{Fe}$ & $\mathrm{H}_{2} \mathrm{O}_{2}$ & Porp & ImH & Arg38 & His42 & \multicolumn{1}{l}{$\mathrm{H}_{2} \mathrm{O}$} \\
\hline $\mathrm{H}_{2} \mathrm{O}_{2}$ complex Doublet & 1.0415 & -0.0006 & -0.0358 & -0.0046 & -0.0003 & 0 & -0.0002 \\
\hline TS H-alpha transfer Doublet & 1.0387 & $-1 \mathrm{E}-04$ & -0.0359 & -0.0027 & $-4.066 \mathrm{E}-20$ & 0 & 0 \\
\hline Cpd 0, conf 1, Doublet & 1.0388 & 0 & -0.0364 & -0.0023 & $1 \mathrm{E}-04$ & -0.0001 & -0.0001 \\
\hline Cpd 0, conf 2, Doublet & 1.0211 & 0.0138 & -0.0387 & 0.0032 & 0.0006 & 0 & 0 \\
\hline Charges & $\mathrm{Fe}$ & $\mathrm{H}_{2} \mathrm{O}_{2}$ & Porp & $\mathrm{ImH}$ & Arg38 & His42 & $\mathrm{H}_{2} \mathrm{O}$ \\
\hline $\mathrm{H}_{2} \mathrm{O}_{2}$ complex Doublet & 1.2667 & 0.3208 & -0.7678 & 0.1888 & 0.8243 & 0.1906 & -0.0234 \\
\hline TS Halpha transfer Doublet & 1.2825 & 0.4033 & -0.8855 & 0.1561 & 0.7994 & 0.2324 & 0.0118 \\
\hline Cpd 0, conf 1, Doublet & 1.3388 & 0.365 & -0.909 & 0.1105 & 0.7489 & 0.3951 & -0.0493 \\
\hline Cpd 0, conf 2, Doublet & 1.2487 & 0.6366 & -1.1003 & 0.1106 & 0.764 & 0.4094 & -0.069 \\
\hline
\end{tabular}

Table S6 : Spin Densities and Charges at the PBE0/B2 level

\begin{tabular}{|l|l|l|l|l|l|r|r|}
\hline Spin densities & $\mathrm{Fe}$ & $\mathrm{H}_{2} \mathrm{O}_{2}$ & Porp & $\mathrm{ImH}$ & Arg38 & His42 & $\mathrm{H}_{2} \mathrm{O}$ \\
\hline $\mathrm{H}_{2} \mathrm{O}_{2}$ complex Doublet & 1.079 & -0.003 & -0.0642 & -0.0115 & -0.0001 & -0.0001 & -0.0001 \\
\hline TS Halpha transfer Doublet & 1.0766 & -0.0038 & -0.063 & -0.0101 & 0.0001 & 0 & 0.0002 \\
\hline Cpd 0, conf 1, Doublet & 1.0775 & -0.0047 & -0.0633 & -0.0093 & $-1 \mathrm{E}-04$ & 0.0001 & -0.0002 \\
\hline Cpd 0, conf 2, Doublet & 1.0557 & 0.0067 & -0.059 & -0.0035 & 0.0004 & 0 & -0.0003 \\
\hline Charges & $\mathrm{Fe}$ & $\mathrm{H}_{2} \mathrm{O}_{2}$ & Porp & $\mathrm{ImH}$ & $\mathrm{Arg} 38$ & $\mathrm{His} 42$ & $\mathrm{H}_{2} \mathrm{O}$ \\
\hline $\mathrm{H}_{2} \mathrm{O}_{2}$ complex Doublet & 1.3138 & 0.3534 & -0.8488 & 0.1733 & 0.8537 & 0.1725 & -0.0179 \\
\hline TS Halpha transfer Doublet & 1.3208 & 0.4352 & -0.9596 & 0.1431 & 0.8273 & 0.211 & 0.0222 \\
\hline Cpd 0, conf 1, Doublet & 1.3815 & 0.3929 & -0.9803 & 0.0993 & 0.7751 & 0.3689 & -0.0374 \\
\hline Cpd 0, conf 2, Doublet & 1.2765 & 0.6751 & -1.167 & 0.0959 & 0.7912 & 0.3828 & -0.0545 \\
\hline
\end{tabular}




\section{Structures}

\section{$\mathrm{H}_{2} \mathrm{O}_{2}$ complex Doublet}

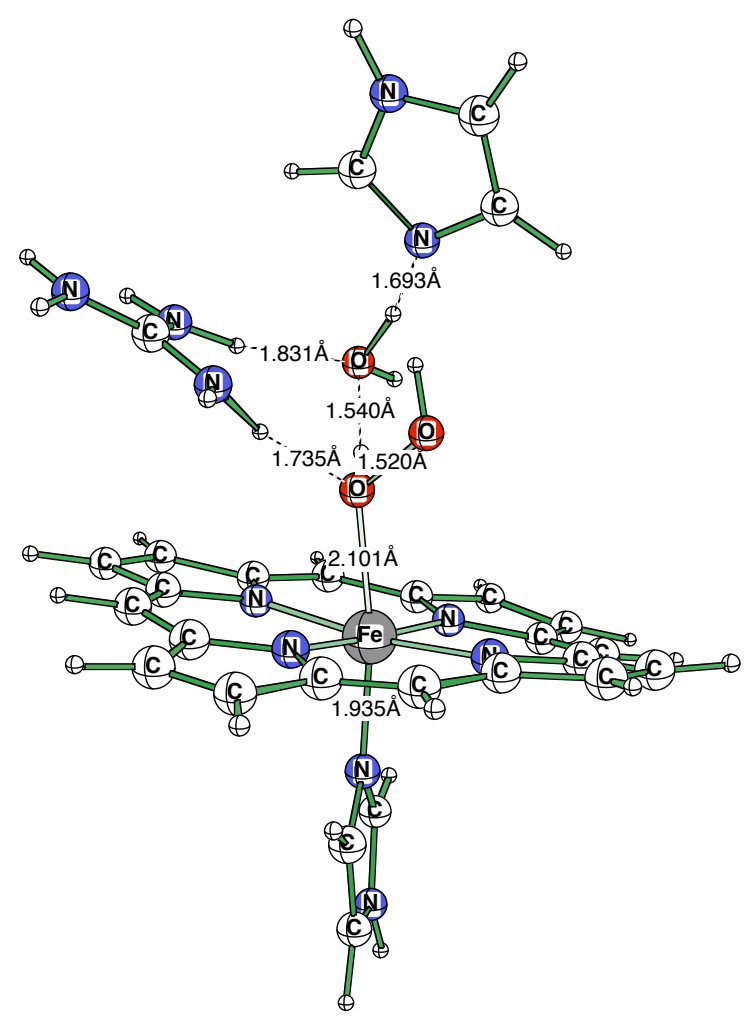

72

$\begin{array}{lrrr}\mathrm{N} & -2.197601 & 1.851547 & 1.485748 \\ \mathrm{H} & -1.555115 & 1.215469 & 0.983200 \\ \mathrm{C} & -3.087982 & 2.596085 & 0.778397 \\ \mathrm{~N} & -4.185425 & 3.146225 & 1.327148 \\ \mathrm{H} & -4.534760 & 2.894714 & 2.252701 \\ \mathrm{H} & -4.817917 & 3.655869 & 0.702042 \\ \mathrm{~N} & -2.826782 & 2.799973 & -0.517532 \\ \mathrm{H} & -1.956421 & 2.490509 & -0.972092 \\ \mathrm{H} & -3.524216 & 3.220413 & -1.126480 \\ \mathrm{~N} & 0.221970 & 5.889481 & 1.273605 \\ \mathrm{H} & -0.265625 & 6.783844 & 1.422363 \\ \mathrm{C} & 1.470993 & 5.544800 & 1.776291 \\ \mathrm{C} & -0.211823 & 4.897018 & 0.462036 \\ \mathrm{H} & -1.145325 & 4.929749 & -0.074493 \\ \mathrm{~N} & 0.702957 & 3.914276 & 0.421356 \\ \mathrm{C} & 1.767670 & 4.315643 & 1.238449 \\ \mathrm{H} & 2.637772 & 3.694702 & 1.368774 \\ \mathrm{~N} & -0.022324 & -5.555832 & -1.225677 \\ \mathrm{H} & 0.123627 & -6.279541 & -1.957306 \\ \mathrm{C} & -0.259430 & -5.786087 & 0.124176 \\ \mathrm{C} & 0.039658 & -4.221375 & -1.436966 \\ \mathrm{H} & 0.238724 & -3.764008 & -2.386917\end{array}$

$\begin{array}{llll}\mathrm{N} & -0.147171 & -3.577637 & -0.268585\end{array}$

C $\quad-0.341583 \quad-4.550735 \quad 0.711899$

H $\quad-0.526169-4.285721 \quad 1.735352$

$\begin{array}{llll}\mathrm{O} & -0.208588 & 0.413284 & 0.240158\end{array}$

$\begin{array}{llll}\mathrm{O} & 0.749512 & 1.188553 & 1.129639\end{array}$

H $\quad-0.167740 \quad 0.953400 \quad-0.647278$

$\mathrm{H} \quad 0.602921 \quad 2.144714 \quad 0.852585$

$\begin{array}{llll}\mathrm{Fe} & -0.036255 & -1.671967 & 0.045120\end{array}$

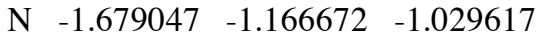

N $1.098785 \quad-1.466721 \quad-1.622421$

$\begin{array}{llll}\mathrm{N} & 1.629715 & -1.925644 & 1.160568\end{array}$

$\begin{array}{llll}\mathrm{N} & -1.132797 & -1.790115 & 1.718124\end{array}$

C $\quad-2.974594 \quad-1.017426 \quad-0.534927$

$\begin{array}{llll}\text { C } & -3.878525 & -0.640060 & -1.615906\end{array}$

C $\quad-3.129242 \quad-0.543411 \quad-2.754578$

C $\quad-1.756409-0.863968-2.389435$

$\begin{array}{llll}\text { C } & 0.658279 & -1.143524 & -2.897751\end{array}$

C $\quad 1.775986-1.188553 \quad-3.830948$

C $2.886673 \quad-1.597534 \quad-3.140366$

C $\quad 2.464340 \quad-1.736954 \quad-1.743073$

$\begin{array}{llll}\text { C } & 2.928574 & -1.977615 & 0.685425\end{array}$

$\begin{array}{llll}\text { C } & 3.881485 & -2.077545 & 1.790848\end{array}$

C $3.153030 \quad-2.151550 \quad 2.945694$

C $\quad 1.741287 \quad-2.084229 \quad 2.537338$

$\begin{array}{llll}\text { C } & -0.693954 & -2.152954 & 2.985764\end{array}$

$\begin{array}{llll}\text { C } & -1.824097 & -2.314407 & 3.878525\end{array}$

C $\quad-2.947311 \quad-1.984131 \quad 3.173325$

$\begin{array}{llll}\text { C } & -2.514130 & -1.654587 & 1.819168\end{array}$

C $\quad-3.340622 \quad-1.219116 \quad 0.789566$

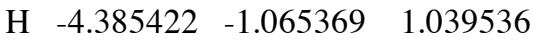

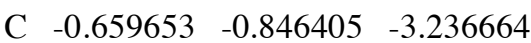

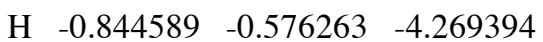

C $3.293503 \quad-1.942322 \quad-0.651306$

$\mathrm{H} \quad 4.350815 \quad-2.018021 \quad-0.841217$

C $\quad 0.637650 \quad-2.252945 \quad 3.360657$

$\mathrm{H} \quad 0.825684 \quad-2.506195 \quad 4.397873$

$\begin{array}{lllll}\text { O } & -0.207413 & 2.260376 & -1.460663\end{array}$

H $\quad 0.295074 \quad 2.936493 \quad-0.898331$

H $\quad 0.327698 \quad 2.058853 \quad-2.279358$

H $-0.375534 \quad-6.783859 \quad 0.547485$

$\mathrm{H} \quad-4.953796-0.494888-1.512451$

$\mathrm{H} \quad-3.481812-0.292770 \quad-3.754868$

H $\quad 1.641464 \quad-1.019867 \quad-4.899216$

H $3.848206 \quad-1.797745 \quad-3.612900$

H $4.953796 \quad-1.991272 \quad 1.615677$

H $3.444183 \quad-2.223663 \quad 3.993408$

H $\quad-1.741074 \quad-2.687439 \quad 4.899216$

H $\quad-3.974808 \quad-1.951569 \quad 3.535431$

H $\quad 1.979691 \quad 6.218140 \quad 2.465958$

$\begin{array}{llll}\mathrm{H} & -2.243912 & 1.641327 & 2.462173\end{array}$ 


\section{TS Halpha transfer Doublet}

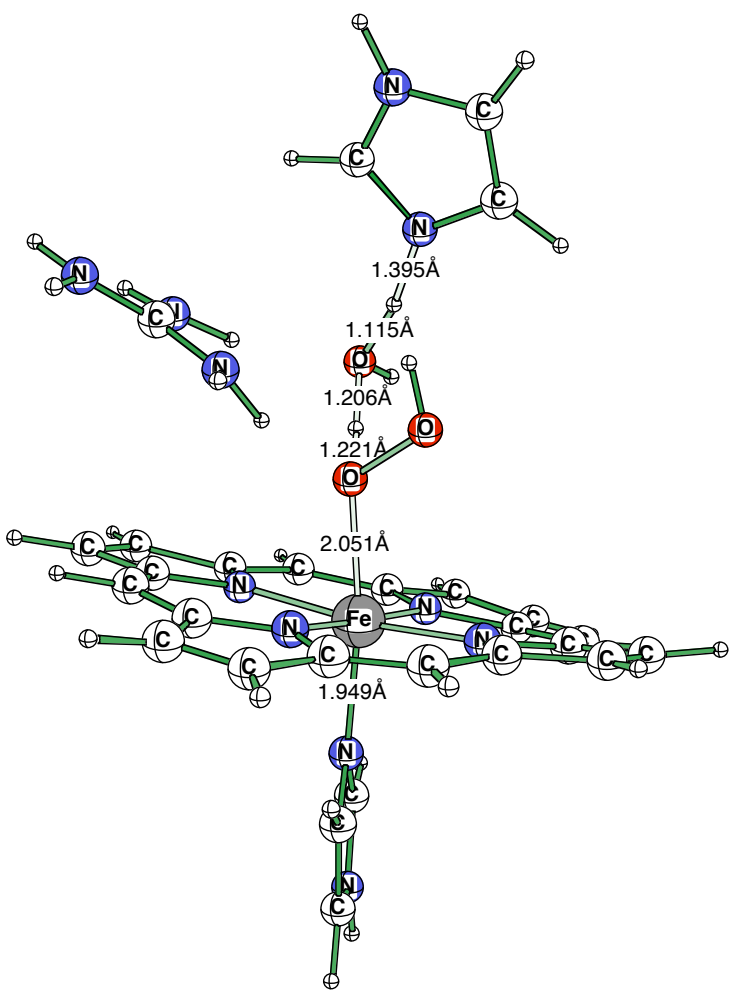

72

$\begin{array}{lrrc}\mathrm{N} & -1.975723 & 1.869034 & 1.529510 \\ \mathrm{H} & -1.401031 & 1.172195 & 1.003204 \\ \mathrm{C} & -2.902740 & 2.603683 & 0.856949 \\ \mathrm{~N} & -3.947556 & 3.202194 & 1.455246 \\ \mathrm{H} & -4.234741 & 2.990677 & 2.410248 \\ \mathrm{H} & -4.615433 & 3.706039 & 0.858932 \\ \mathrm{~N} & -2.722534 & 2.749268 & -0.463135 \\ \mathrm{H} & -1.890671 & 2.396149 & -0.943832 \\ \mathrm{H} & -3.448654 & 3.142548 & -1.056946 \\ \mathrm{~N} & 0.453796 & 5.892868 & 0.907639 \\ \mathrm{H} & -0.010635 & 6.789474 & 1.112915 \\ \mathrm{C} & 1.725876 & 5.512985 & 1.326553 \\ \mathrm{C} & -0.049408 & 4.935730 & 0.097565 \\ \mathrm{H} & -1.015137 & 4.987122 & -0.374924 \\ \mathrm{~N} & 0.842331 & 3.940369 & -0.020248 \\ \mathrm{C} & 1.964569 & 4.290161 & 0.743484 \\ \mathrm{H} & 2.827591 & 3.647690 & 0.802124 \\ \mathrm{~N} & -0.201065 & -5.613632 & -1.117920 \\ \mathrm{H} & -0.132385 & -6.359512 & -1.838928 \\ \mathrm{C} & -0.324265 & -5.805038 & 0.252731 \\ \mathrm{C} & -0.134308 & -4.285919 & -1.369080 \\ \mathrm{H} & -0.017639 & -3.858200 & -2.346832 \\ \mathrm{~N} & -0.206955 & -3.608170 & -0.208481 \\ \mathrm{C} & -0.332123 & -4.552078 & 0.811493 \\ \mathrm{H} & -0.422821 & -4.257996 & 1.838287 \\ \mathrm{O} & -0.155563 & 0.359161 & 0.205124 \\ \mathrm{O} & 0.899628 & 1.033005 & 1.073532 \\ \mathrm{H} & -0.081116 & 1.085678 & -0.772888 \\ \mathrm{H} & 0.683167 & 1.993530 & 0.968216\end{array}$

$\begin{array}{llll}\text { Fe } & -0.042175 & -1.682068 & 0.043121\end{array}$

$\mathrm{N}-1.768066-1.205338 \quad-0.902283$

$\begin{array}{llll}\mathrm{N} & 0.947952 & -1.559662 & -1.723587\end{array}$

N $1.711853 \quad-1.952362 \quad 1.018616$

$\begin{array}{llll}\mathrm{N} & -0.995087 & -1.756836 & 1.806519\end{array}$

C $\quad-3.010880 \quad-1.012161 \quad-0.303543$

C $\quad-4.001694-0.660461 \quad-1.318359$

C $\quad-3.352890 \quad-0.632233 \quad-2.520493$

$\begin{array}{llll}\text { C } & -1.957962 & -0.960510 & -2.259003\end{array}$

C $\quad 0.398163 \quad-1.288132 \quad-2.967300$

C $\quad 1.429047 \quad-1.383118 \quad-3.992996$

C $2.594604 \quad-1.771957 \quad-3.385269$

C $2.294846 \quad-1.847519 \quad-1.951324$

$\begin{array}{llll}\text { C } & 2.964539 & -2.026810 & 0.438095\end{array}$

C $4.006973 \quad-2.121185 \quad 1.461227$

$\begin{array}{llll}\text { C } & 3.378677 & -2.164688 & 2.674789\end{array}$

$\begin{array}{llll}\text { C } & 1.936951 & -2.084946 & 2.383011\end{array}$

$\begin{array}{llll}\text { C } & -0.454025 & -2.096725 & 3.039688\end{array}$

C $\quad-1.505661 \quad-2.213501 \quad 4.031311$

C $\quad-2.679108 \quad-1.877716 \quad 3.416901$

C $\quad-2.359543 \quad-1.590118 \quad 2.021286$

$\begin{array}{llll}\text { C } & -3.263947 & -1.164627 & 1.054352\end{array}$

H $-4.280594 \quad-0.985672 \quad 1.389587$

C $-0.942932-0.998444 \quad-3.203476$

H $\quad-1.223862 \quad-0.791824 \quad-4.229843$

C $3.213577-2.025208 \quad-0.926605$

H $\quad 4.250961 \quad-2.110260 \quad-1.205093$

C $\quad 0.903931 \quad-2.216080 \quad 3.300903$

H $\quad 1.175034 \quad-2.450790 \quad 4.323807$

$\begin{array}{lllll}\text { O } & -0.028183 & 2.085922 & -1.444794\end{array}$

H $\quad 0.476089 \quad 2.902283 \quad-0.877029$

H $\quad 0.438232 \quad 1.892563 \quad-2.309036$

H $\quad-0.419769 \quad-6.789459 \quad 0.710709$

$\mathrm{H} \quad-5.061554-0.491348 \quad-1.128418$

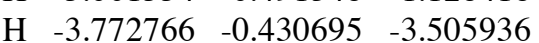

H $\quad 1.192764 \quad-1.258835 \quad-5.049744$

H $\quad 3.507629 \quad-2.008636 \quad-3.931519$

H $\quad 5.061554 \quad-2.053741 \quad 1.194427$

H $3.755157 \quad-2.225479 \quad 3.695862$

$\mathrm{H} \quad-1.340927 \quad-2.565201 \quad 5.049744$

$\mathrm{H} \quad-3.671402 \quad-1.816986 \quad 3.863708$

H $\quad 2.286682 \quad 6.176941 \quad 1.984283$

H $\quad-1.969421 \quad 1.698975 \quad 2.514862$ 


\section{Cpd 0, conf 1, Doublet}

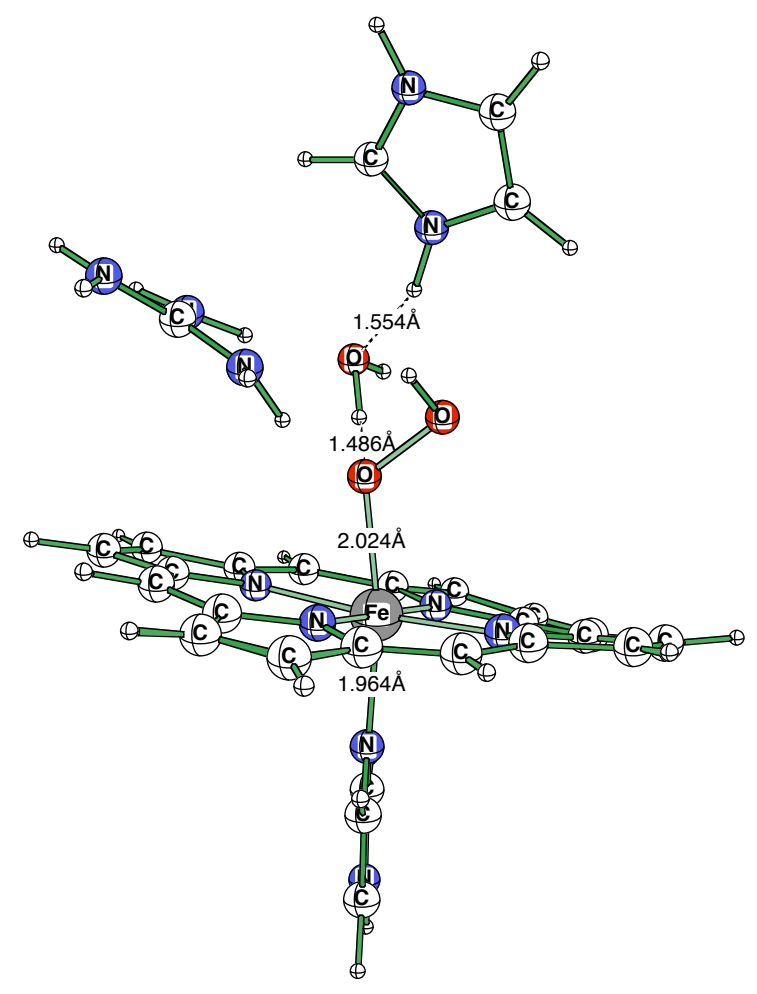

72

$\begin{array}{lrrc}\mathrm{N} & -1.915131 & 1.853928 & 1.499298 \\ \mathrm{H} & -1.399277 & 1.123383 & 0.926300 \\ \mathrm{C} & -2.904297 & 2.575638 & 0.891327 \\ \mathrm{~N} & -3.919601 & 3.151001 & 1.561783 \\ \mathrm{H} & -4.142761 & 2.930649 & 2.531952 \\ \mathrm{H} & -4.636353 & 3.635010 & 1.008392 \\ \mathrm{~N} & -2.815247 & 2.728439 & -0.433929 \\ \mathrm{H} & -1.996277 & 2.408401 & -0.981277 \\ \mathrm{H} & -3.603500 & 3.086426 & -0.969009 \\ \mathrm{~N} & 0.429749 & 5.911804 & 0.611893 \\ \mathrm{H} & -0.031189 & 6.809647 & 0.829071 \\ \mathrm{C} & 1.706223 & 5.525848 & 1.015945 \\ \mathrm{C} & -0.111588 & 4.953201 & -0.158569 \\ \mathrm{H} & -1.091339 & 4.978394 & -0.603561 \\ \mathrm{~N} & 0.777191 & 3.956131 & -0.252380 \\ \mathrm{C} & 1.921082 & 4.281570 & 0.472626 \\ \mathrm{H} & 2.762482 & 3.613556 & 0.531143 \\ \mathrm{~N} & -0.225037 & -5.666443 & -1.045944 \\ \mathrm{H} & -0.196075 & -6.424576 & -1.755508 \\ \mathrm{C} & -0.255798 & -5.833694 & 0.332489 \\ \mathrm{C} & -0.185043 & -4.340851 & -1.322220 \\ \mathrm{H} & -0.138367 & -3.929382 & -2.312103 \\ \mathrm{~N} & -0.184738 & -3.643524 & -0.171646 \\ \mathrm{C} & -0.236237 & -4.570374 & 0.868057 \\ \mathrm{H} & -0.261627 & -4.258972 & 1.893768 \\ \mathrm{O} & -0.206802 & 0.315857 & 0.157623 \\ \mathrm{O} & 0.918900 & 1.158463 & 0.766006 \\ \mathrm{H} & -0.293350 & 1.198334 & -1.035324 \\ \mathrm{H} & 0.421173 & 1.739059 & 1.389755\end{array}$

Fe $\quad-0.031998 \quad-1.697281 \quad 0.039520$

$\begin{array}{llll}\mathrm{N} & -1.828278 & -1.286453 & -0.805695\end{array}$

N $\quad 0.841232 \quad-1.611099 \quad-1.791290$

$\begin{array}{llll}\mathrm{N} & 1.785599 & -1.936508 & 0.905823\end{array}$

$\begin{array}{llll}\mathrm{N} & -0.873230 & -1.770782 & 1.857376\end{array}$

$\begin{array}{llll}\text { C } & -3.033829 & -1.098938 & -0.136810\end{array}$

$\begin{array}{llll}\text { C } & -4.089935 & -0.776459 & -1.095306\end{array}$

C $\quad-3.515244 \quad-0.761078-2.334290$

C $-2.102051 \quad-1.063721 \quad-2.150345$

$\begin{array}{llll}\text { C } & 0.211838 & -1.361755 & -3.000015\end{array}$

C $\quad 1.174988-1.464310 \quad-4.089432$

C $\quad 2.379242 \quad-1.839218 \quad-3.551910$

C $2.172943 \quad-1.891861 \quad-2.099747$

$\begin{array}{llll}\text { C } & 2.996124 & -2.015717 & 0.246094\end{array}$

C $4.104523 \quad-2.077271 \quad 1.200485$

$\begin{array}{llll}\text { C } & 3.556915 & -2.100128 & 2.453781\end{array}$

$\begin{array}{llll}\text { C } & 2.097977 & -2.038818 & 2.253738\end{array}$

C $\quad-0.248444 \quad-2.074615 \quad 3.059586$

C $\quad-1.234924 \quad-2.186127 \quad 4.117889$

$\begin{array}{llll}\text { C } & -2.450684 & -1.880157 & 3.572235\end{array}$

C $\quad-2.224182 \quad-1.621429 \quad 2.152863$

C $\quad-3.196365 \quad-1.229019 \quad 1.237488$

H $-4.192001-1.058334 \quad 1.633209$

C $-1.147034 \quad-1.096771 \quad-3.154602$

H $\quad-1.493546 \quad-0.910904-4.164536$

C $3.156616-2.043182 \quad-1.132874$

H $\quad 4.174240 \quad-2.129990 \quad-1.475677$

C $\quad 1.125549 \quad-2.163300 \quad 3.238815$

H $\quad 1.465042 \quad-2.372055 \quad 4.247971$

$\begin{array}{lllll}\text { O } & -0.376495 & 2.071457 & -1.587189\end{array}$

H $\quad 0.516129 \quad 3.066162 \quad-0.795242$

H $\quad 0.057739 \quad 1.916595 \quad-2.472031$

H $\quad-0.316666 \quad-6.809647 \quad 0.813919$

H $-5.138458-0.614410 \quad-0.845718$

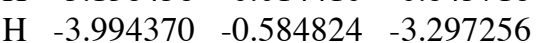

H $\quad 0.866608 \quad-1.361755 \quad-5.129776$

H $\quad 3.255981 \quad-2.083557 \quad-4.151611$

H $\quad 5.138474 \quad-2.006439 \quad 0.863037$

H $3.998886 \quad-2.137665 \quad 3.449371$

H $\quad-1.002365 \quad-2.517670 \quad 5.129776$

H $\quad-3.414017 \quad-1.817368 \quad 4.078247$

H $\quad 2.293594 \quad 6.203857 \quad 1.635086$

H $\quad-1.850250 \quad 1.683945 \quad 2.482544$ 


\section{Cpd 0, conf2, Doublet}

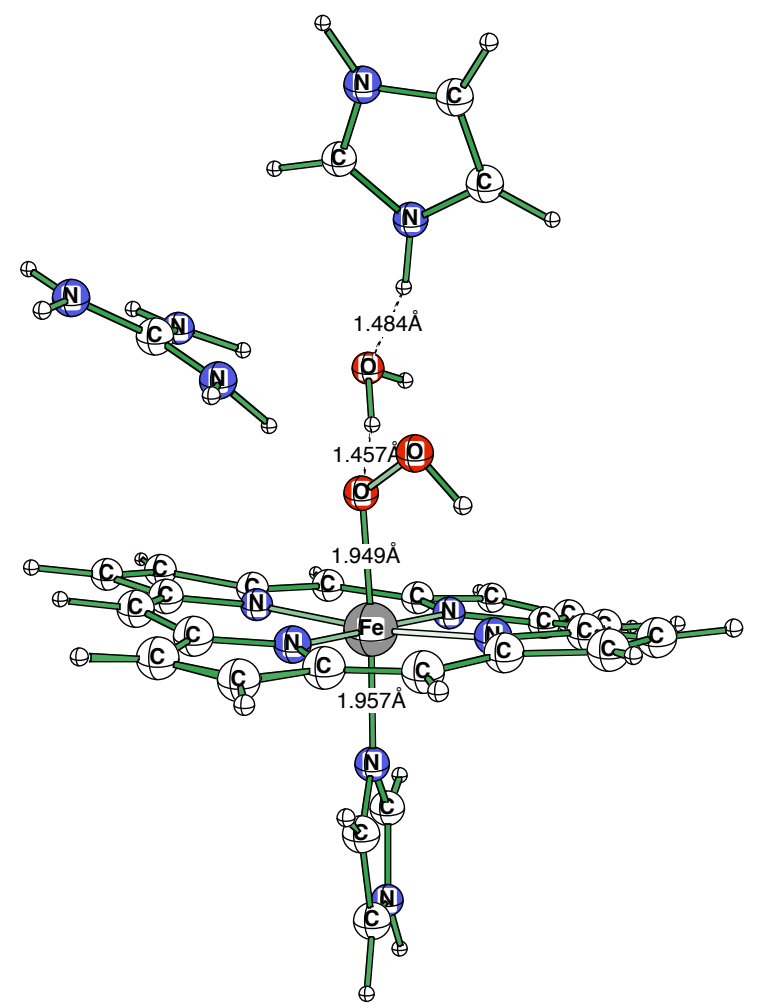

72

$\begin{array}{lrrr}\mathrm{N} & -2.223724 & 1.769699 & 1.226807 \\ \mathrm{H} & -1.498016 & 1.178558 & 0.755417 \\ \mathrm{C} & -3.126587 & 2.447769 & 0.477554 \\ \mathrm{~N} & -4.280609 & 2.943817 & 0.962250 \\ \mathrm{H} & -4.652420 & 2.690536 & 1.877380 \\ \mathrm{H} & -4.919968 & 3.401962 & 0.303665 \\ \mathrm{~N} & -2.794479 & 2.660477 & -0.806061 \\ \mathrm{H} & -1.872650 & 2.374619 & -1.170593 \\ \mathrm{H} & -3.477493 & 2.989227 & -1.483337 \\ \mathrm{~N} & -0.199966 & 5.960754 & 1.061249 \\ \mathrm{H} & -0.771057 & 6.804413 & 1.227341 \\ \mathrm{C} & 1.065399 & 5.719788 & 1.584915 \\ \mathrm{C} & -0.509705 & 5.004211 & 0.167374 \\ \mathrm{H} & -1.424225 & 4.941132 & -0.396835 \\ \mathrm{~N} & 0.521790 & 4.152695 & 0.111053 \\ \mathrm{C} & 1.517960 & 4.576950 & 0.979660 \\ \mathrm{H} & 2.456818 & 4.066116 & 1.076599 \\ \mathrm{~N} & 0.261688 & -5.597595 & -1.174515 \\ \mathrm{H} & 0.471970 & -6.325989 & -1.885956 \\ \mathrm{C} & -0.018845 & -5.815063 & 0.167267 \\ \mathrm{C} & 0.263779 & -4.264175 & -1.408524 \\ \mathrm{H} & 0.472794 & -3.814819 & -2.360855 \\ \mathrm{~N} & -0.005005 & -3.609680 & -0.265244 \\ \mathrm{C} & -0.188248 & -4.574005 & 0.723312 \\ \mathrm{H} & -0.432007 & -4.302887 & 1.731033 \\ \mathrm{O} & -0.192429 & 0.251205 & 0.277069 \\ \mathrm{O} & 0.521912 & 0.759674 & 1.512878 \\ \mathrm{H} & -0.004166 & 1.272522 & -0.744217\end{array}$

$\begin{array}{llll}\mathrm{H} & 1.177734 & 0.021667 & 1.628860\end{array}$

Fe $\quad-0.035233 \quad-1.676819 \quad 0.036880$

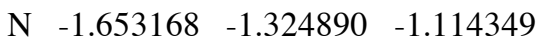

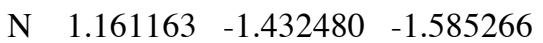

$\begin{array}{llll}\mathrm{N} & 1.626099 & -1.790329 & 1.247345\end{array}$

$\begin{array}{llll}\mathrm{N} & -1.172058 & -1.914505 & 1.663269\end{array}$

C $\quad-2.961823 \quad-1.208832 \quad-0.666092$

C $\quad-3.845840 \quad-0.884766 \quad-1.786453$

$\begin{array}{llll}\text { C } & -3.063797 & -0.800171 & -2.901627\end{array}$

$\begin{array}{llll}\text { C } & -1.691177 & -1.056915 & -2.476761\end{array}$

C $\quad 0.754211 \quad-1.187988 \quad-2.885544$

C $\quad 1.912674 \quad-1.164230 \quad-3.770462$

C $3.021118-1.448776 \quad-3.015411$

C $2.547714 \quad-1.562958 \quad-1.630356$

$\begin{array}{llll}\text { C } & 2.949417 & -1.718231 & 0.825989\end{array}$

$\begin{array}{llll}\text { C } & 3.852890 & -1.795044 & 1.968887\end{array}$

$\begin{array}{llll}\text { C } & 3.086868 & -1.982971 & 3.089127\end{array}$

$\begin{array}{llll}\text { C } & 1.693130 & -2.025131 & 2.624634\end{array}$

$\begin{array}{llll}\text { C } & -0.761887 & -2.232544 & 2.951141\end{array}$

C $\quad-1.919678-2.399155 \quad 3.811066$

C $\quad-3.025833 \quad-2.113190 \quad 3.061188$

$\begin{array}{llll}\text { C } & -2.557327 & -1.809296 & 1.712814\end{array}$

$\begin{array}{llll}\text { C } & -3.361099 & -1.404190 & 0.651642\end{array}$

H $\quad-4.416916-1.279663 \quad 0.867233$

C $\quad-0.567184 \quad-1.004501 \quad-3.286743$

H $\quad-0.730972 \quad-0.793259-4.337158$

C $3.351059 \quad-1.635498 \quad-0.498383$

H $\quad 4.415787 \quad-1.592804 \quad-0.660110$

C $\quad 0.558395 \quad-2.281998 \quad 3.384171$

H $\quad 0.709381 \quad-2.543747 \quad 4.425446$

$\begin{array}{llll}\text { O } & -0.046890 & 2.132751 & -1.328140\end{array}$

H $\quad 0.450562 \quad 3.255402 \quad-0.494766$

H $\quad 0.528061 \quad 2.006378 \quad-2.133591$

$\begin{array}{llll}\mathrm{H} & -0.105087 & -6.804398 & 0.616455\end{array}$

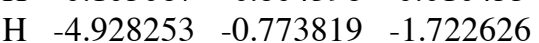

H $-3.378555 \quad-0.597351 \quad-3.925232$

H $1.804092 \quad-1.046204 \quad-4.848511$

H $\quad 4.012405 \quad-1.613998 \quad-3.437302$

H $\quad 4.928253 \quad-1.667221 \quad 1.845291$

H $\quad 3.335999 \quad-2.065445 \quad 4.146988$

H $-1.863953-2.728653 \quad 4.848495$

H $\quad-4.066238 \quad-2.094604 \quad 3.385468$

H $\quad 1.501083 \quad 6.402206 \quad 2.314651$

H $\quad-2.319351 \quad 1.499115 \quad 2.184677$ 


\section{QM/MM scan : OO bond breaking from Cpd 0, conf2}

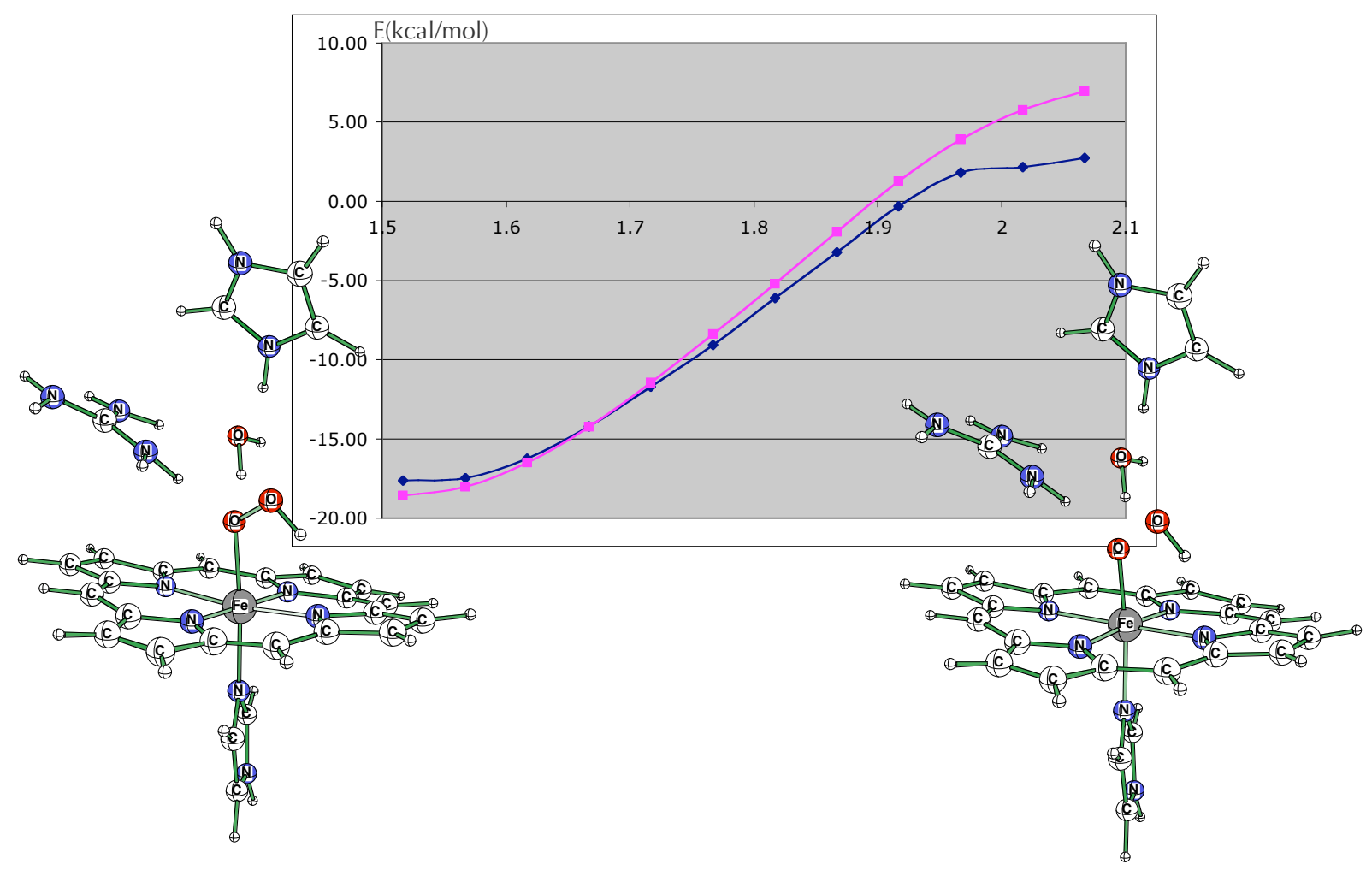

Starting from Cpd 0, conf2, a QM/MM scan was done along the OO bond coordinate, at the $\mathrm{B} 3 \mathrm{LYP} / \mathrm{B} 1$ level. The curve in pink corresponds to the QM/MM values, and in blue to the $\mathrm{QM}$ values. As can be seen from the figure above, the $\mathrm{OH}$ radical created is not able to complete the formation of Cpd I. 


\section{QM/MM scan : $H$ bonding to O-beta}

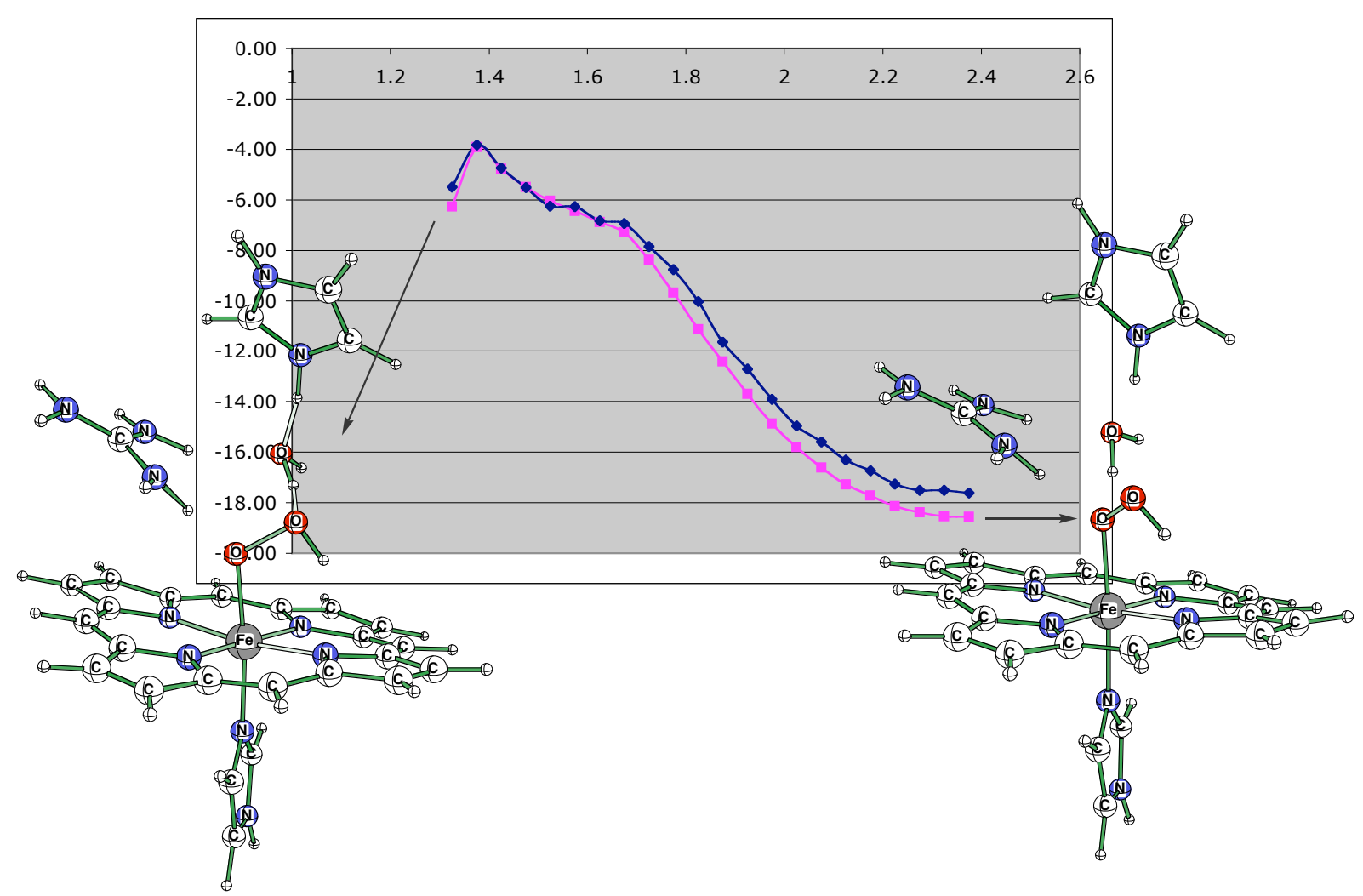

Since there is no hydrogen in a good position, an attempt was made to create a more reactive conformation. The curve in pink corresponds to the QM/MM values, and in blue to the QM values. Starting from Conformation 2 of Cpd 0, a scan was run in order to create an hydrogen bond between the water molecule (see figure above) and the O-beta of $\mathrm{OOH}$. Indeed, this new conformation was found and is $-8.39 \mathrm{kcal} / \mathrm{mol}$ more stable than the $\mathrm{H}_{2} \mathrm{O}_{2}$ complex. We will call this conformation, conf3 


\section{QM/MM scan : OO bond breaking from Cpd 0, conf3}

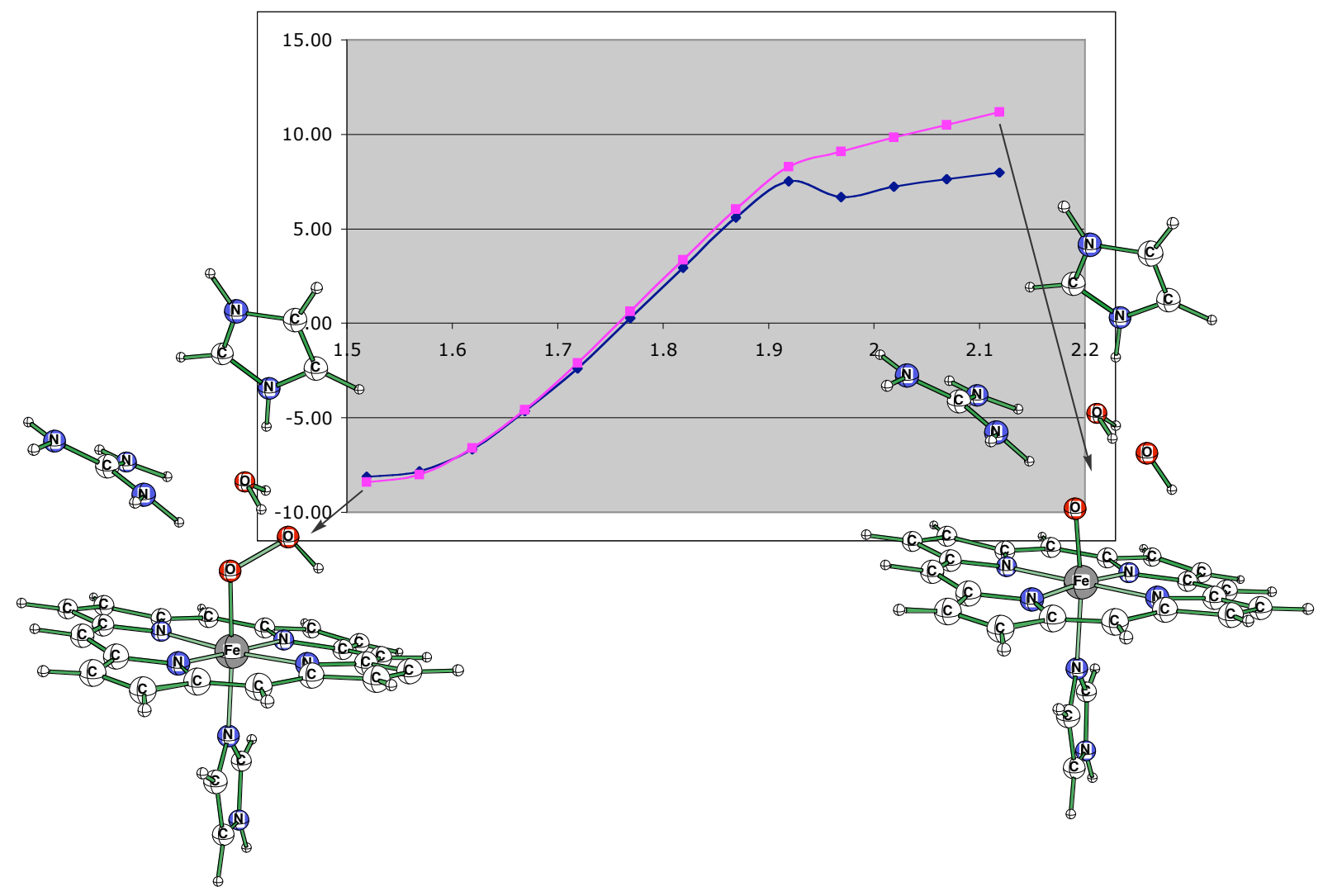

Starting from Cpd 0, conf3, a QM/MM scan was done along the OO bond coordinate, at the $\mathrm{B} 3 \mathrm{LYP} / \mathrm{B} 1$ level. The curve in pink corresponds to the QM/MM values, and in blue to the $\mathrm{QM}$ values. As can be seen from the figure above, the $\mathrm{OH}$ radical created is not able to complete the formation of Cpd I. 


\section{QM/MM scan : $\mathrm{H}$ transfer to create $\mathrm{Fe}-\mathrm{OOH}_{2}$}

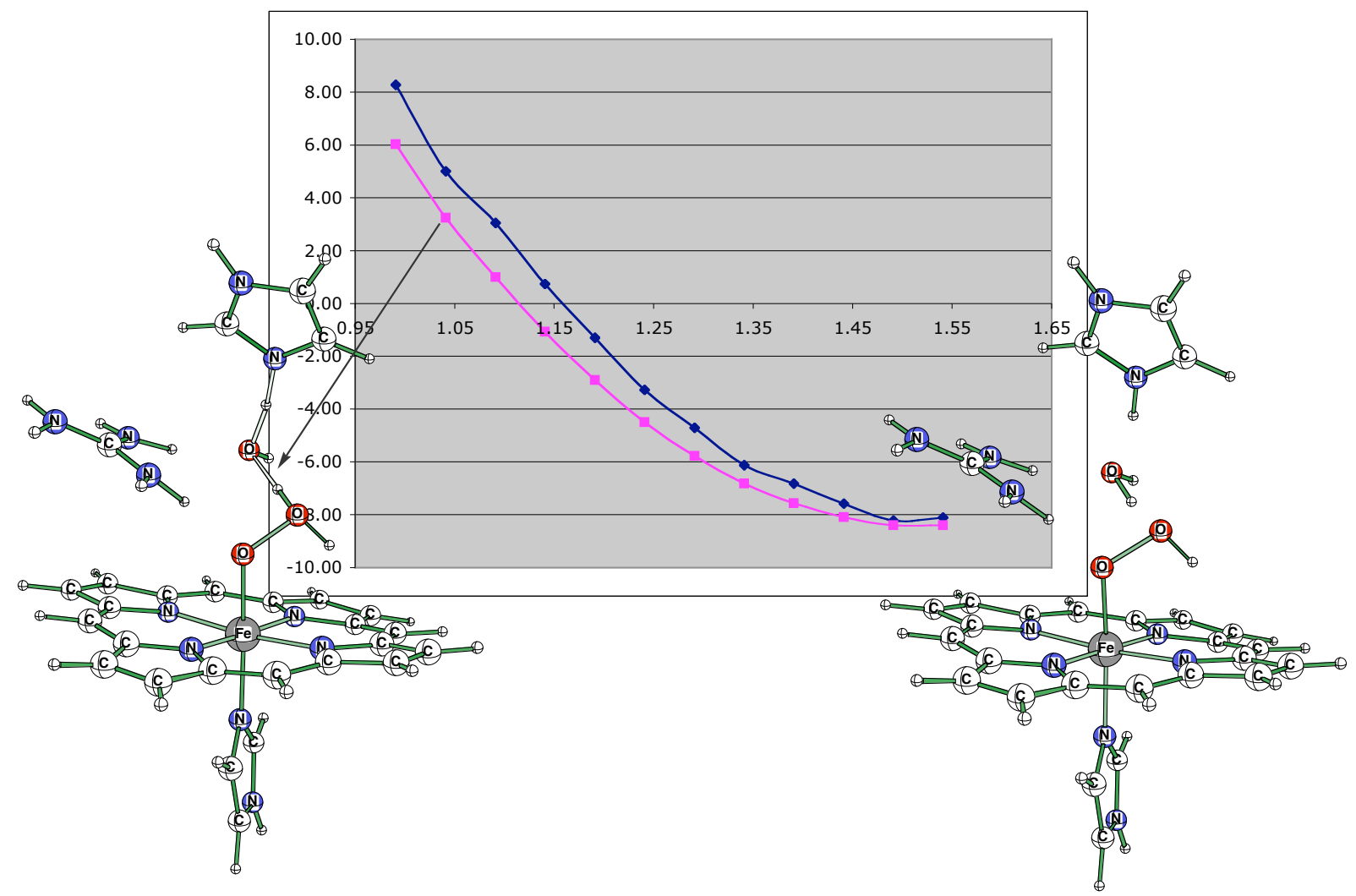

Another scan was done in order to create the $\mathrm{Fe}-\mathrm{OOH}_{2}$ species, by scanning the $\mathrm{OH}$ coordinate instead of the $\mathrm{OO}$ coordinate. Curve in pink corresponds to the QM/MM values, and in blue to the QM values. The proton transfer doesn't lead to a minimum for a distance of $1.041 \AA$ A. No H transfer can therefore occur. 


\section{Energetic Decomposition Conf.1/Conf. 2}

Table S7: absolute (a.u.) and relative ( $\mathrm{kcal} / \mathrm{mol})$ energies for the two conformations of Compound 0 in different environnement. Geometries are taken from the QM/MM full optimization. Level : B3LYP/BS1//CHARMM

\begin{tabular}{|l|l|l|l|r|r|r|}
\hline & Conf 1 & \multicolumn{1}{|c|}{ Conf2 } & & \\
\hline & $\mathrm{QM}$ & $\mathrm{QM} / \mathrm{MM}$ & $\mathrm{QM}$ & $\mathrm{QM} / \mathrm{MM}$ & $\Delta \mathrm{E}(\mathrm{QM})$ & $\Delta \mathrm{E}(\mathrm{QM} / \mathrm{MM})$ \\
\hline QM/MM normal (B3LYP/B1) & -1997.872042 & -2079.34288 & -1997.897031 & -2079.368012 & -15.68 & -15.77 \\
\hline QM/MM W2407 in MM & -1921.466067 & -2002.918594 & -1921.493253 & -2002.943387 & -17.06 & -15.56 \\
\hline QM/MM W2407 in MM, no PC(W2407) & -1921.387533 & -2002.827303 & -1921.412197 & -2002.848946 & -15.48 & -13.58 \\
\hline QM/MM Arg38 in MM & -1792.027449 & -1874.006288 & -1792.042335 & -1874.023527 & -9.34 & -10.82 \\
\hline QM/MM Arg38 in MM, no PC ${ }^{\text {a }}$ & -1792.022756 & -1873.48493 & -1792.042949 & -1873.508316 & -12.67 & -14.68 \\
\hline QM/MM Arg38\&W2407 in MM & -1715.598302 & -1797.579461 & -1715.619221 & -1797.596653 & -13.13 & -10.79 \\
\hline QM/MM Arg38\&W2407 in MM, no PC & -1715.536446 & -1797.487594 & -1715.551905 & -1797.500803 & -9.70 & -8.29 \\
\hline
\end{tabular}

a. no PC means point charges are deleted.

Table S8 : absolute (a.u.) and relative ( $\mathrm{kcal} / \mathrm{mol})$ energies of the QM models calculated at the $\mathrm{B} 3 \mathrm{LYP} / \mathrm{SV}(\mathrm{P})$ level. Geometries are taken from the QM/MM full optimization and residues are progessively removed.

\begin{tabular}{|c|c|c|c|}
\hline decomposition QM model & Conf 1 & Conf 2 & $\Delta \mathrm{E}$ \\
\hline full system & -3136.563465 & -3136.580299 & -10.56 \\
\hline $\operatorname{Arg} 38$ & -2930.998508 & -2931.008907 & -6.53 \\
\hline Water & -3060.154632 & -3060.170137 & -9.73 \\
\hline Arg38 and Water & -2854.600647 & -2854.609381 & -5.48 \\
\hline His42 & -2910.170703 & -2910.183924 & -8.30 \\
\hline His42 and Water & -2833.797319 & -2833.815055 & -11.13 \\
\hline His42 and Arg38 & -2704.499353 & -2704.505952 & -4.14 \\
\hline His42 and Arg38 and Water & -2628.151101 & -2628.162338 & -7.05 \\
\hline
\end{tabular}

Table S9: Decomposition of the MM contributions for the relative stability of the two conformations of Compound 0. Level : B3LYP/B1:CHARMM

\begin{tabular}{|l|r|r|r|}
\hline Comparison QM \& QM/MM & Conf 1 & Conf 2 & $\Delta \mathrm{E}$ \\
\hline QM/MM & -2079.34288 & -2079.368012 & -15.77 \\
\hline QM part of QM/MM & -1997.872042 & -1997.897031 & -15.68 \\
\hline Pure QM (all PC ${ }^{\mathrm{a}}$ removed) & -1997.369502 & -1997.387278 & -11.15 \\
\hline
\end{tabular}

a. PC means point charges are deleted.

From this table, it appears that the contribution of MM part itself is small $(0.09 \mathrm{kcal} / \mathrm{mol})$, but the influence of the MM part on the QM region through point charges is quite important (4.53 $\mathrm{kcal} / \mathrm{mol}$ ), nearly one third of the total difference. 


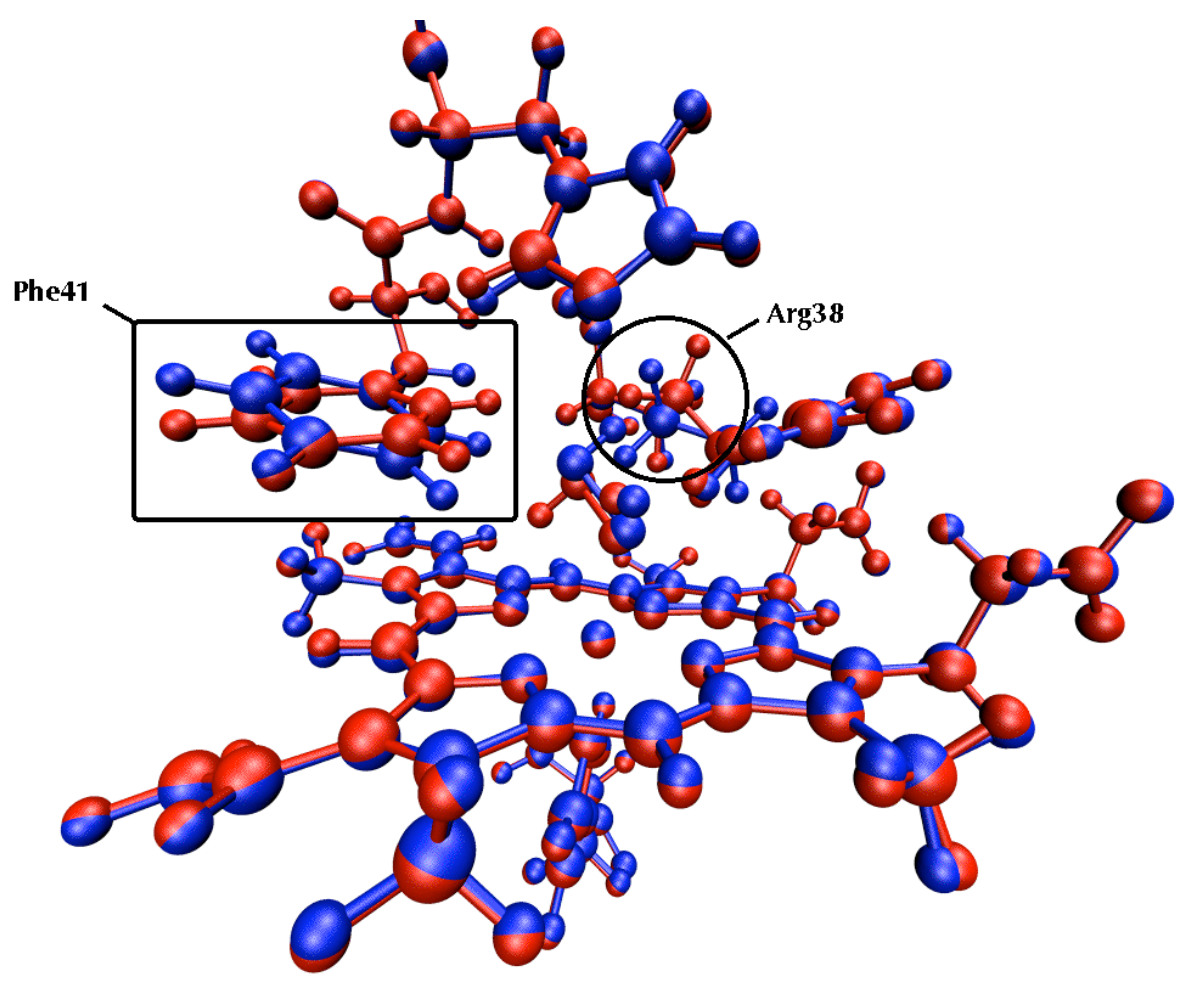

Figure S5. View of Compound 0 Conf. 1 (blue) and Conf. 2 (red), including the surrounding part treated at the MM level. Phe41 adopts a diffrent conformation in Conf. 2 in order to avoid the $\mathrm{OH}$ part of $\mathrm{OOH}$. In the same manner, alkyl chain of Arg38 is reorganized in Conf. 2. Effect of these rearrangements for the MM energy is weak, but influence on the QM region is quite important. 


\section{QM/MM Data without a water molecule}

These data ate extracted from the following paper, for comparison purposes :

E. Derat \& S. Shaik, J. Phys. Chem. B, 2006, 110, 10526-10533.

\section{Energies}

Table S10 : Absolute Energies with B3LYP, Doublet State

\begin{tabular}{|c|c|c|c|c|}
\hline Structure & $\mathrm{QM}(\mathrm{B} 1)$ & $\mathrm{QM} / \mathrm{MM}(\mathrm{B} 1)$ & $\mathrm{QM}(\mathrm{B} 2)$ & $\mathrm{QM} / \mathrm{MM}(\mathrm{B} 2)$ \\
\hline \multicolumn{5}{|c|}{ Conformation 1} \\
\hline \multicolumn{5}{|c|}{$\mathrm{H}_{2} \mathrm{O}_{2}$ Complex -1921.5067729-1996.9895626-3062.2535124-3137.7363067 } \\
\hline TS & -1921.487289 & $3-1996.9670447$ & 3062.22417 & -3137.7038771 \\
\hline Cpd 0 & -1921.506 & 2-1996.99439 & 3062.24 & -3137.7338292 \\
\hline \multicolumn{5}{|c|}{ Conformation 2} \\
\hline \multicolumn{5}{|c|}{$\mathrm{H}_{2} \mathrm{O}_{2}$ Complex - } \\
\hline TS & -1921.49 & 1970.909 & 02.2. & -3137.7084942 \\
\hline Cpd 0 & -1921.499579 & 7-1996.9845270 & 3062.23624 & -3137.7211643 \\
\hline
\end{tabular}

Table S11 : Relative Energies with B3LYP, Doublet State Structure $\quad \mathrm{QM}(\mathrm{B} 1) \mathrm{QM} / \mathrm{MM}(\mathrm{B} 1) \mathrm{QM}(\mathrm{B} 2) \mathrm{QM} / \mathrm{MM}(\mathrm{B} 2)$

\section{Conformation 1}

$\begin{array}{crrrr}\mathrm{H}_{2} \mathrm{O}_{2} \text { Complex } & 0.00 & 0.00 & 0.00 & 0.00 \\ \text { TS } & 12.23 & 14.13 & 18.41 & 20.35 \\ \text { Cpd } 0 & 0.22 & -3.03 & 4.96 & 1.55\end{array}$

Conformation 2

\begin{tabular}{crrrr}
$\mathrm{H}_{2} \mathrm{O}_{2}$ Complex & -0.20 & 5.41 & -2.01 & 3.64 \\
$\mathrm{TS}$ & 8.34 & 12.41 & 13.33 & 17.45 \\
$\mathrm{Cpd} \mathrm{0}$ & 4.51 & 3.16 & 10.84 & 9.50 \\
\hline
\end{tabular}

Table S12 : Absolute and Relative Energies with B3LYP, Quartet State

\begin{tabular}{ccrrr} 
Structure & QM(B1) & QM/MM(B1) & QM(B2) & QM/MM(B2) \\
\hline $\mathrm{H}_{2} \mathrm{O}_{2}$ Complex & $-1921.50091-1996.986694$ & -3062.253836 & -3137.739584 \\
TS & $-1921.489025-1996.969925$ & -3062.2311782 & -3137.7120383 \\
Cpd 0 & $-1921.49762-1996.984124$ & -3062.236455 & -3137.722913 \\
$\mathrm{H}_{2} \mathrm{O}_{2}$ Complex & 3.68 & 1.80 & -0.20 & -2.06 \\
TS & 11.14 & 12.32 & 14.01 & 15.23 \\
Cpd 0 & 5.74 & 3.41 & 10.70 & 8.40 \\
\hline
\end{tabular}




\section{Spin densities \& Charges}

Table S13 : Spin densities B3LYP/B1

\begin{tabular}{|c|c|}
\hline Structure & $\rho(\mathrm{Fe}) \rho(\mathrm{H} 2 \mathrm{O} 2) \rho(\operatorname{ImH}) \rho$ (Por) $\rho$ (His) $\rho$ (Arg) \\
\hline \multicolumn{2}{|c|}{ Conformation 1, Doublet } \\
\hline $\mathrm{H}_{2} \mathrm{O}_{2}$ Complex & $1.0682-0.0043-0.0063-0.0575-0.0001$ \\
\hline TS & $1.0769-0.0041-0.0147-0.0577-0.00050 .0001$ \\
\hline Compound 0 & $\begin{array}{llll}1.0372 & 0.0128 & 0.0019 & -0.054\end{array}$ \\
\hline \multicolumn{2}{|c|}{ Conformation 2,Doublet } \\
\hline $\mathrm{H}_{2} \mathrm{O}_{2}$ Complex & $1.1075-0.001 \quad-0.0114-0.0951$ \\
\hline TS & $1.0938-0.0061-0.0207-0.067-0.00010 .0001$ \\
\hline Cpd 0 & 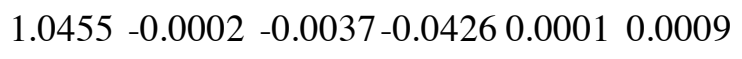 \\
\hline \multicolumn{2}{|l|}{ Quartet } \\
\hline $\mathrm{H}_{2} \mathrm{O}_{2}$ Complex & $\begin{array}{llllll}0.017 & 0.1693 & 0.0168 & 0.0000 & 0.0000\end{array}$ \\
\hline TS & $\begin{array}{llll}2.8209 & 0.0294 & 0.161-0.0112 & 0.001-0.0011\end{array}$ \\
\hline Cpd 0 & $\begin{array}{llllll}2.9075 & 0.0346 & 0.0223 & 0.0335 & 0.0000 & 0.0022\end{array}$ \\
\hline
\end{tabular}

Table S14 : Charges B3LYP/B1

\begin{tabular}{|c|c|}
\hline Structure & $\mathrm{Q}(\mathrm{Fe}) \mathrm{Q}\left(\mathrm{H}_{2} \mathrm{O}_{2}\right) \mathrm{Q}(\mathrm{ImH}) \mathrm{Q}($ Por $) \mathrm{Q}(\mathrm{His}) \mathrm{Q}(\mathrm{Arg})$ \\
\hline \multicolumn{2}{|c|}{ Conformation 1, Doublet } \\
\hline $\mathrm{H}_{2} \mathrm{O}_{2}$ Complex & $\begin{array}{llll}0.7094 & 0.0487 & 0.4363 & -0.23630 .14070 .9012\end{array}$ \\
\hline TS & 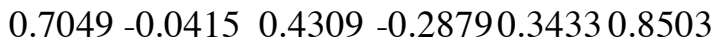 \\
\hline Cpd 0 & 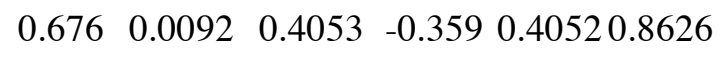 \\
\hline \multicolumn{2}{|c|}{ Conformation 2, Doublet } \\
\hline $\mathrm{H}_{2} \mathrm{O}_{2}$ Complex & $0.7069-0.00820 .4478-0.18930 .12560 .9172$ \\
\hline TS & $\begin{array}{llllll}0.725 & -0.0981 & 0.4215 & -0.274 & 0.3985 & 0.8271\end{array}$ \\
\hline Cpd 0 & $\begin{array}{llll}0.6973 & -0.0118 & 0.3937 & -0.32820 .39430 .8547\end{array}$ \\
\hline \multicolumn{2}{|l|}{ Quartet } \\
\hline $\mathrm{H}_{2} \mathrm{O}_{2}$ Complex & $\begin{array}{llllll}0.846 & 0.0314 & 0.3183 & -0.242 & 0.145 & 0.9012\end{array}$ \\
\hline $\mathrm{TS}$ & 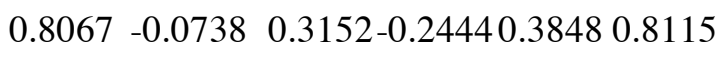 \\
\hline Cpd 0 & $\begin{array}{llll}0.8457 & -0.017 & 0.3515-0.42050 .3978 & 0.8422\end{array}$ \\
\hline
\end{tabular}




\section{Structures}

\section{$\mathrm{H}_{2} \mathrm{O}_{2}$ Complex ; Conformation 1 ; Doublet}

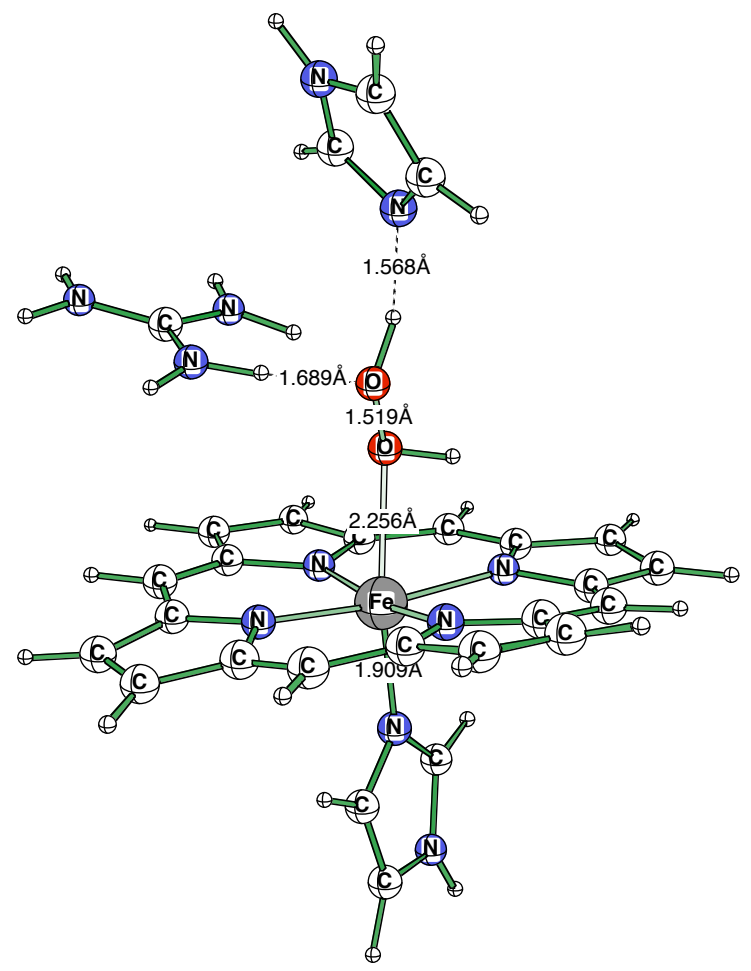

69

$\begin{array}{lrrr}\mathrm{N} & -2.737289 & 1.905060 & 0.395096 \\ \mathrm{H} & -1.729538 & 1.741837 & 0.566406 \\ \mathrm{C} & -3.179794 & 2.495499 & -0.739456 \\ \mathrm{~N} & -4.462555 & 2.866547 & -0.902115 \\ \mathrm{H} & -5.194885 & 2.600296 & -0.250183 \\ \mathrm{H} & -4.748184 & 3.288284 & -1.796280 \\ \mathrm{~N} & -2.271820 & 2.758789 & -1.683792 \\ \mathrm{H} & -1.314087 & 2.420517 & -1.619904 \\ \mathrm{H} & -2.506958 & 3.226151 & -2.568512 \\ \mathrm{~N} & -0.878036 & 5.903519 & 1.483643 \\ \mathrm{H} & -1.495300 & 6.724121 & 1.418884 \\ \mathrm{C} & -0.067535 & 5.575653 & 2.563461 \\ \mathrm{C} & -0.670197 & 4.999863 & 0.494232 \\ \mathrm{H} & -1.168457 & 5.022034 & -0.461197 \\ \mathrm{~N} & 0.243332 & 4.100967 & 0.894424 \\ \mathrm{C} & 0.632233 & 4.455597 & 2.187698 \\ \mathrm{H} & 1.363541 & 3.880798 & 2.728485 \\ \mathrm{~N} & 0.752014 & -5.303329 & -1.561920 \\ \mathrm{H} & 1.115234 & -5.960785 & -2.297791 \\ \mathrm{C} & 0.068985 & -5.681747 & -0.412857 \\ \mathrm{C} & 0.816025 & -3.959396 & -1.609238 \\ \mathrm{H} & 1.302917 & -3.392715 & -2.378082\end{array}$

$\begin{array}{rrrr}\mathrm{N} & 0.194641 & -3.448196 & -0.524826 \\ \mathrm{C} & -0.275101 & -4.520966 & 0.231247 \\ \mathrm{H} & -0.814362 & -4.366669 & 1.145721 \\ \mathrm{O} & -0.123871 & 1.585434 & 1.066635 \\ \mathrm{O} & 0.049362 & 0.658981 & -0.123779 \\ \mathrm{H} & 0.178085 & 2.539963 & 0.759018 \\ \mathrm{H} & 1.033722 & 0.542160 & -0.271255 \\ \mathrm{Fe} & -0.000107 & -1.596481 & -0.102951 \\ \mathrm{~N} & -0.924057 & -1.077957 & -1.808807 \\ \mathrm{~N} & 1.800644 & -1.121643 & -0.932175 \\ \mathrm{~N} & 0.906479 & -1.817917 & 1.696976 \\ \mathrm{~N} & -1.766266 & -1.839111 & 0.803009 \\ \mathrm{C} & -2.298294 & -0.987915 & -2.021439 \\ \mathrm{C} & -2.570770 & -0.568375 & -3.393906 \\ \mathrm{C} & -1.360092 & -0.393082 & -3.999969 \\ \mathrm{C} & -0.335007 & -0.681091 & -3.004715 \\ \mathrm{C} & 2.034653 & -0.755203 & -2.261429 \\ \mathrm{C} & 3.460754 & -0.688461 & -2.503571 \\ \mathrm{C} & 4.112335 & -1.094650 & -1.368362 \\ \mathrm{C} & 3.073685 & -1.347061 & -0.373230 \\ \mathrm{C} & 2.269470 & -1.771118 & 1.942200 \\ \mathrm{C} & 2.547363 & -1.936020 & 3.367111 \\ \mathrm{C} & 1.349197 & -2.150650 & 3.991409 \\ \mathrm{C} & 0.330383 & -2.104721 & 2.932846 \\ \mathrm{C} & -1.985245 & -2.338989 & 2.084702 \\ \mathrm{C} & -3.385300 & -2.666824 & 2.264450 \\ \mathrm{C} & -4.035110 & -2.290710 & 1.122162 \\ \mathrm{C} & -3.022446 & -1.785980 & 0.204681 \\ \mathrm{C} & -3.261581 & -1.291473 & -1.070923 \\ \mathrm{H} & -4.300064 & -1.195267 & -1.369720 \\ \mathrm{C} & 1.032349 & -0.536331 & -3.199829 \\ \mathrm{H} & 1.343994 & -0.215637 & -4.187256 \\ \mathrm{C} & 3.252487 & -1.606110 & 0.975021 \\ \mathrm{H} & 4.264023 & -1.633240 & 1.342377 \\ \mathrm{C} & -1.015457 & -2.411667 & 3.073456 \\ \mathrm{H} & -1.344437 & -2.760544 & 4.045792 \\ \mathrm{H} & -0.127548 & -6.724121 & -0.162292 \\ \mathrm{H} & -3.563049 & -0.478378 & -3.835785 \\ \mathrm{H} & -1.146606 & -0.128357 & -5.035431 \\ \mathrm{H} & 3.856400 & -0.385986 & -3.473053 \\ \mathrm{H} & 5.194901 & -1.204773 & -1.305801 \\ \mathrm{H} & 3.552567 & -1.799774 & 3.765793 \\ \mathrm{H} & 1.084152 & -2.316788 & 5.035431 \\ \mathrm{H} & -3.790237 & -3.186508 & 3.132751 \\ \mathrm{H} & -5.104752 & -2.331345 & 0.916687 \\ \mathrm{H} & -0.078720 & 6.170212 & 3.476776 \\ \mathrm{H} & -3.284958 & 1.511200 & 1.133255\end{array}$

$\begin{array}{lllll}\mathrm{N} & 0.194641 & -3.448196 & -0.524826\end{array}$

$\begin{array}{llll}\mathrm{O} & -0.123871 & 1.585434 & 1.066635\end{array}$

$\begin{array}{llll}\mathrm{O} & 0.049362 & 0.658981 & -0.123779\end{array}$

$\begin{array}{llll}\mathrm{H} & 0.178085 & 2.539963 & 0.759018\end{array}$

$\begin{array}{llll}\mathrm{H} & 1.033722 & 0.542160 & -0.271255\end{array}$

$\begin{array}{llll}\text { Fe } & -0.000107 & -1.596481 & -0.102951\end{array}$

N $1.800644-1.121643-0.932175$

$\begin{array}{llll}\mathrm{N} & 0.906479 & -1.817917 & 1.696976\end{array}$

N $-1.766266-1.839111 \quad 0.803009$

$\begin{array}{llll}\text { C } & -2.570770 & -0.568375 & -3.393906\end{array}$

$\begin{array}{llll}\text { C } & -1.360092 & -0.393082 & -3.999969\end{array}$

$\begin{array}{llll}\text { C } & -0.335007 & -0.681091 & -3.004715\end{array}$

$\begin{array}{llll}\text { C } 3.460754 & -0.688461 & -2.503571\end{array}$

$\begin{array}{llll}\text { C } & 4.112335 & -1.094650 & -1.368362\end{array}$

$\begin{array}{llll}\text { C } & 3.073685 & -1.347061 & -0.373230\end{array}$

$\begin{array}{llll}\text { C } & 2.269470 & -1.771118 & 1.942200\end{array}$

$\begin{array}{llll}\text { C } & 0.330383 & -2.104721 & 2.932846\end{array}$

$\begin{array}{llll}\text { C } & -1.985245 & -2.338989 & 2.084702\end{array}$

$\begin{array}{llll}\text { C } & -3.385300 & -2.666824 & 2.264450\end{array}$

$\begin{array}{llll}\text { C } & -4.035110 & -2.290710 & 1.122162\end{array}$

$\begin{array}{llll}\text { C } & -3.022446 & -1.785980 & 0.204681\end{array}$

H $-4.300064-1.195267-1.369720$

$\begin{array}{llll}\text { C } & 1.032349 & -0.536331 & -3.199829\end{array}$

$\begin{array}{llll}\mathrm{H} & 1.343994 & -0.215637 & -4.187256\end{array}$

$\begin{array}{llll}\text { C } & 3.252487 & -1.606110 & 0.975021\end{array}$

$\begin{array}{llll}\mathrm{H} & 4.264023 & -1.633240 & 1.342377\end{array}$

$\begin{array}{llll}\text { C } & -1.015457 & -2.411667 & 3.073456\end{array}$

$\begin{array}{llll}\mathrm{H} & -1.344437 & -2.760544 & 4.045792\end{array}$

$\begin{array}{llll}\mathrm{H} & -0.127548 & -6.724121 & -0.162292\end{array}$

$\begin{array}{llll}\mathrm{H} & -3.563049 & -0.478378 & -3.835785\end{array}$

\begin{tabular}{llll}
$\mathrm{H}$ & -1.146606 & -0.128357 & -5.03543 \\
\hline & 3.856400 & -0.385986 & -3.473053
\end{tabular}

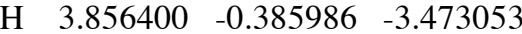

H $\quad 5.194901 \quad-1.204773-1.305801$

$\begin{array}{lllll}\mathrm{H} & 3.552567 & -1.799774 & 3.765793\end{array}$

$\mathrm{H} \quad 1.084152 \quad-2.316788 \quad 5.035431$

$\begin{array}{llll}\mathrm{H} & -3.790237 & -3.186508 & 3.132751\end{array}$

$\begin{array}{llll}\mathrm{H} & -0.078720 & 6.170212 & 3.476776\end{array}$

$\mathrm{H} \quad-3.284958 \quad 1.511200 \quad 1.133255$ 


\section{TS ; Conformation 1 : Doublet}

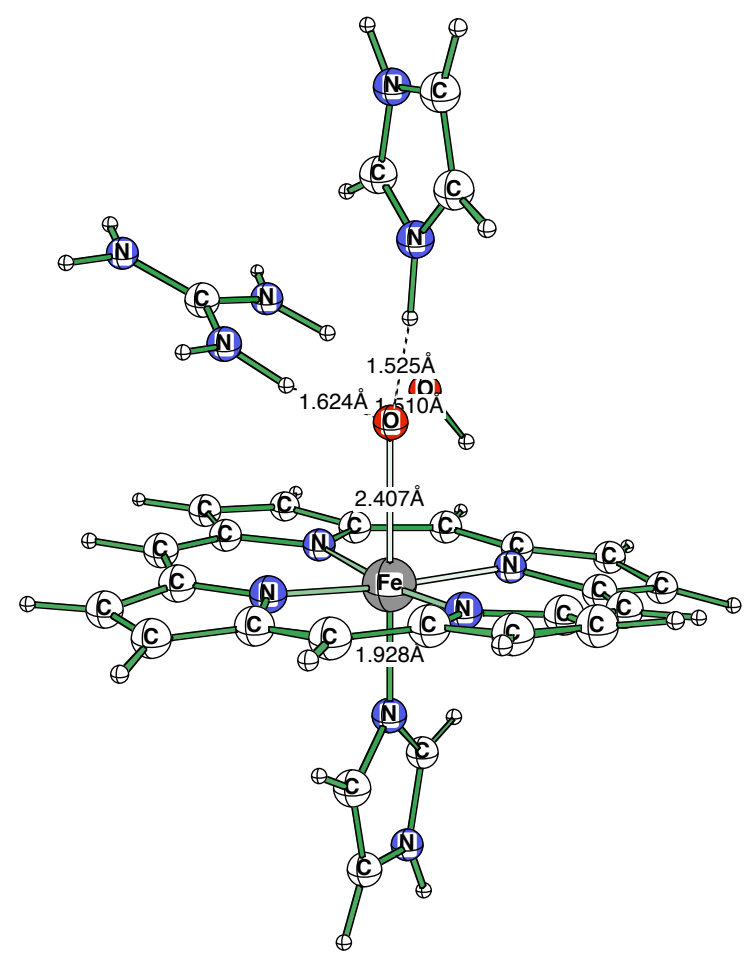

69

$\begin{array}{cccc}\mathrm{N} & -2.353622 & 2.004242 & 0.173630 \\ \mathrm{H} & -1.464645 & 1.428406 & 0.233749 \\ \mathrm{C} & -2.705826 & 2.685638 & -0.940750 \\ \mathrm{~N} & -3.868790 & 3.365067 & -1.044403 \\ \mathrm{H} & -4.633286 & 3.196960 & -0.399490 \\ \mathrm{H} & -4.109787 & 3.828094 & -1.932495 \\ \mathrm{~N} & -1.793335 & 2.738052 & -1.915024 \\ \mathrm{H} & -0.904037 & 2.228851 & -1.817261 \\ \mathrm{H} & -1.973526 & 3.186752 & -2.823822 \\ \mathrm{~N} & 0.029999 & 5.608139 & 0.915497 \\ \mathrm{H} & -0.305710 & 6.502426 & 0.559250 \\ \mathrm{C} & 0.693970 & 5.421432 & 2.129822 \\ \mathrm{C} & -0.148895 & 4.404785 & 0.316284 \\ \mathrm{H} & -0.620682 & 4.246429 & -0.641144 \\ \mathrm{~N} & 0.363159 & 3.452713 & 1.121887 \\ \mathrm{C} & 0.886353 & 4.068588 & 2.247147 \\ \mathrm{H} & 1.322693 & 3.496948 & 3.036758 \\ \mathrm{~N} & 0.269836 & -5.235794 & -1.482254 \\ \mathrm{H} & 0.501923 & -5.959259 & -2.208923 \\ \mathrm{C} & -0.361725 & -5.499527 & -0.273254 \\ \mathrm{C} & 0.473511 & -3.906143 & -1.587341 \\ \mathrm{H} & 0.957855 & -3.424942 & -2.415024 \\ \mathrm{~N} & -0.006454 & -3.294662 & -0.482651 \\ \mathrm{C} & -0.529205 & -4.287125 & 0.345123 \\ \mathrm{H} & -0.979477 & -4.045853 & 1.288223 \\ \mathrm{O} & 0.009903 & 0.888535 & 0.646698 \\ \mathrm{O} & 0.516769 & 1.376022 & -0.689285 \\ \mathrm{H} & 0.281570 & 2.348160 & 0.993958 \\ \mathrm{H} & 1.114502 & 0.639542 & -0.976212 \\ \mathrm{Fe} & -0.000443 & -1.420120 & -0.032578\end{array}$

$\begin{array}{lrrr}\mathrm{N} & -1.022507 & -0.870605 & -1.709442 \\ \mathrm{~N} & 1.747681 & -1.172592 & -1.083603 \\ \mathrm{~N} & 1.033676 & -1.743835 & 1.649826 \\ \mathrm{~N} & -1.699661 & -1.527435 & 0.986465 \\ \mathrm{C} & -2.402573 & -0.728104 & -1.836426 \\ \mathrm{C} & -2.754822 & -0.368225 & -3.210175 \\ \mathrm{C} & -1.586304 & -0.309067 & -3.914124 \\ \mathrm{C} & -0.510559 & -0.605865 & -2.978195 \\ \mathrm{C} & 1.891891 & -0.923447 & -2.453659 \\ \mathrm{C} & 3.278763 & -1.070663 & -2.844910 \\ \mathrm{C} & 3.987213 & -1.484543 & -1.746674 \\ \mathrm{C} & 3.031326 & -1.526566 & -0.637009 \\ \mathrm{C} & 2.410706 & -1.789398 & 1.761032 \\ \mathrm{C} & 2.807434 & -1.902466 & 3.164017 \\ \mathrm{C} & 1.661530 & -1.991806 & 3.905365 \\ \mathrm{C} & 0.551041 & -1.912384 & 2.941681 \\ \mathrm{C} & -1.849533 & -1.930099 & 2.308289 \\ \mathrm{C} & -3.255066 & -2.094437 & 2.618561 \\ \mathrm{C} & -3.964127 & -1.717148 & 1.511993 \\ \mathrm{C} & -2.989807 & -1.379684 & 0.482239 \\ \mathrm{C} & -3.299179 & -0.937271 & -0.798782 \\ \mathrm{H} & -4.350845 & -0.788177 & -1.018768 \\ \mathrm{C} & 0.837708 & -0.617218 & -3.304581 \\ \mathrm{H} & 1.090866 & -0.381805 & -4.331573 \\ \mathrm{C} & 3.310013 & -1.749084 & 0.701859 \\ \mathrm{H} & 4.346161 & -1.850616 & 0.978317 \\ \mathrm{C} & -0.802872 & -2.061630 & 3.214005 \\ \mathrm{H} & -1.074982 & -2.319229 & 4.231567 \\ \mathrm{H} & -0.652328 & -6.502426 & 0.039429 \\ \mathrm{H} & -3.761902 & -0.212143 & -3.596695 \\ \mathrm{H} & -1.440826 & -0.113205 & -4.976349 \\ \mathrm{H} & 3.595200 & -0.907471 & -3.875015 \\ \mathrm{H} & 5.044601 & -1.746445 & -1.784714 \\ \mathrm{H} & 3.854462 & -1.813156 & 3.453201 \\ \mathrm{H} & 1.485992 & -2.091980 & 4.976349 \\ \mathrm{H} & -3.624908 & -2.528931 & 3.547211 \\ \mathrm{H} & -5.044601 & -1.646561 & 1.387283 \\ \mathrm{H} & 0.931656 & 6.235031 & 2.814835 \\ \mathrm{H} & -2.890869 & 1.876358 & 1.007217\end{array}$




\section{Compound 0 Conformation 1 Doublet}

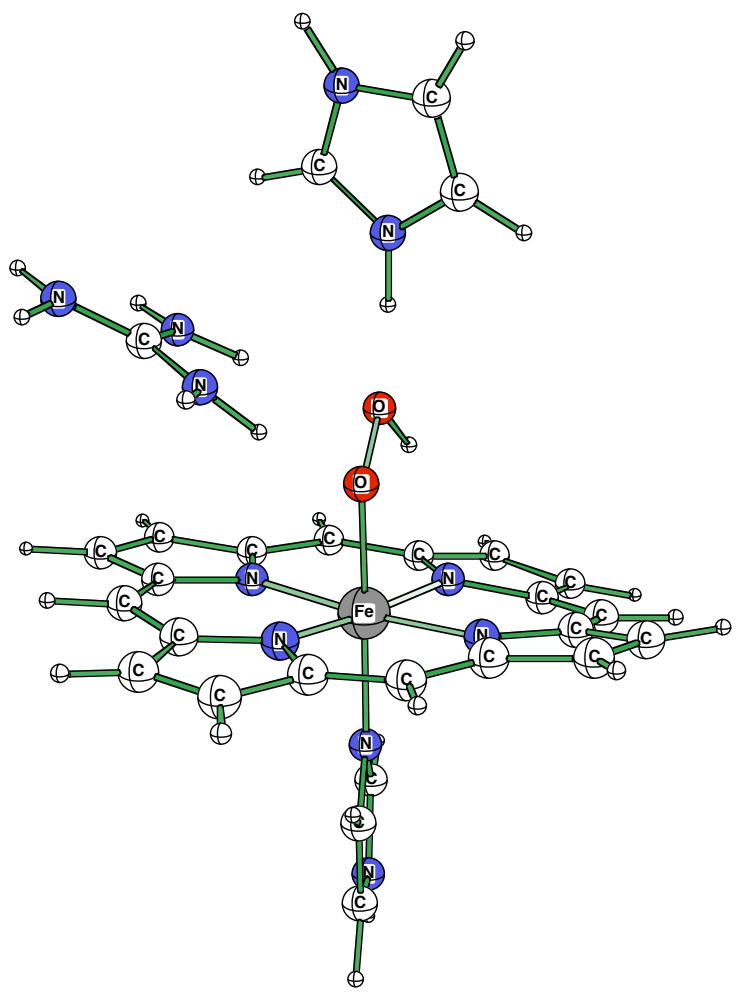

69

$\mathrm{N} \quad-4.401000-0.630000-2.639000$

H $\quad-3.744000-1.445000-2.450000$

C $-5.210000-0.558000-3.719000$

$\mathrm{N} \quad-6.2110000 .344000-3.834000$

H $\quad-6.5730000 .833000-3.020000$

$\mathrm{H} \quad-6.8300000 .308000-4.656000$

$\mathrm{N} \quad-4.923000-1.371000-4.743000$

$\mathrm{H} \quad-4.142000-2.026000-4.679000$

$\mathrm{H} \quad-5.535000-1.475000-5.563000$

$\mathrm{N} \quad-1.8410001 .943000-5.486000$

H $\quad-2.2400002 .701000-6.059000$

C $\quad-0.6060001 .988000-4.835000$

C $-2.4180000 .742000-5.275000$

H $\quad-3.3660000 .414000-5.671000$

$\mathrm{N} \quad-1.5810000 .019000-4.501000$

C $\quad-0.4480000 .775000-4.219000$

H $\quad 0.3560000 .389000-3.623000$

N $\quad-3.509000-7.9040000 .228000$

H $\quad-3.716000-8.9300000 .152000$

C $-3.500000-7.1800001 .414000$

C $-3.285000-7.057000-0.801000$

H $\quad-3.224000-7.350000-1.831000$

N $\quad-3.132000-5.803000-0.327000$

C $-3.263000-5.8760001 .061000$

H $\quad-3.184000-5.0000001 .674000$

O $-2.481000-2.357000-2.055000$

O $\quad-2.194000-2.373000-3.558000$
H $\quad-1.777000-0.950000-4.092000$

H $-1.938000-3.319000-3.711000$

FE $-2.798000-4.138000-1.300000$

N $-4.459000-4.290000-2.463000$

$\mathrm{N} \quad-1.748000-5.160000-2.739000$

$\mathrm{N}-1.099000-3.869000-0.233000$

$\mathrm{N} \quad-3.797000-3.0570000 .050000$

C $\quad-5.708000-3.743000-2.188000$

C $-6.651000-4.082000-3.254000$

C $\quad-5.966000-4.830000-4.168000$

C $-4.595000-4.940000-3.685000$

C $-2.257000-5.748000-3.897000$

C $-1.250000-6.598000-4.507000$

C $-0.146000-6.596000-3.696000$

C $\quad-0.450000-5.673000-2.598000$

C $\quad 0.150000-4.347000-0.572000$

C $1.170000-3.8030000 .328000$

C $\quad 0.523000-3.0330001 .254000$

C $-0.908000-3.0990000 .904000$

C $-3.307000-2.5540001 .246000$

C $-4.386000-1.9500002 .004000$

C $\quad-5.516000-2.0290001 .240000$

C $\quad-5.149000-2.7350000 .016000$

C $-6.002000-2.997000-1.052000$

H $\quad-7.013000-2.614000-0.966000$

C $\quad-3.563000-5.591000-4.349000$

H $\quad-3.807000-6.048000-5.300000$

C $\quad 0.421000-5.228000-1.614000$

H $\quad 1.448000-5.546000-1.686000$

C $-1.967000-2.5490001 .616000$

$\mathrm{H} \quad-1.732000-2.0790002 .565000$

h $\quad-3.672763-7.6338692 .389819$

h $-7.704410-3.804402-3.290526$

h $-6.347014-5.303854-5.072631$

h $-1.462966-7.160200-5.416200$

h $0.730491-7.218026-3.877424$

h $2.229652-3.9697280 .134595$

h $0.879681-2.4419282 .097492$

h $-4.268190-1.5733083 .020017$

h $-6.508377-1.6314651 .452652$

h $\quad 0.0218222 .878985-4.842250$

h $-4.453256-0.067092-1.814151$ 


\section{H2O2 Complex ; Conformation 2 ; Doublet}

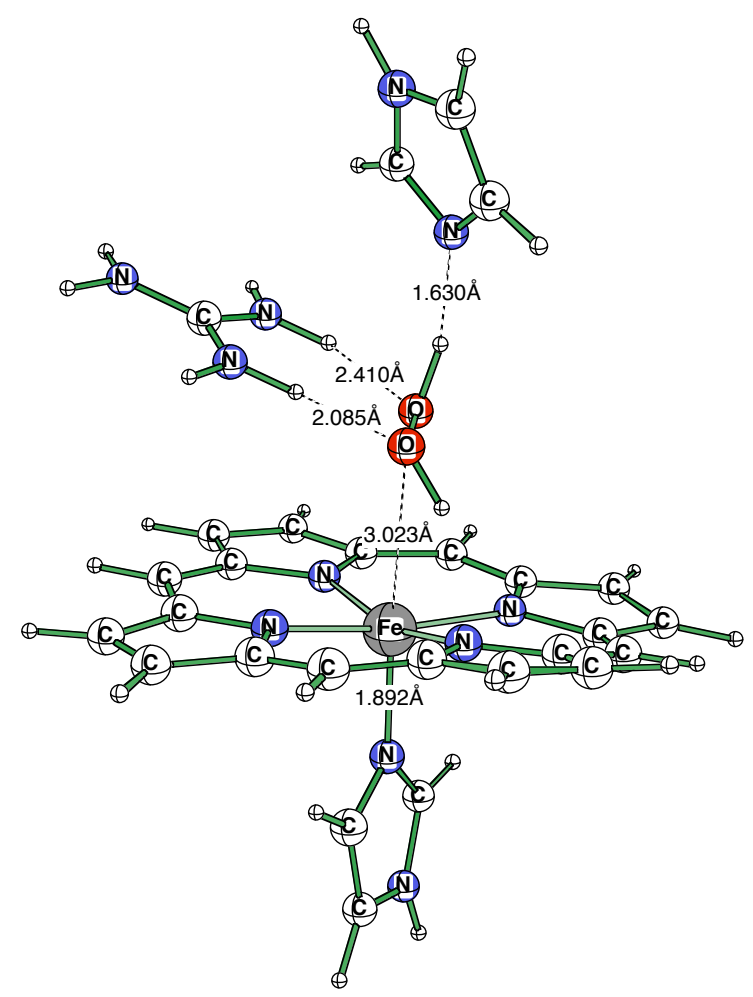

69
$\begin{array}{llll}\mathrm{N} & -2.298462 & 2.061127 & 0.299850\end{array}$
$\begin{array}{llll}\mathrm{H} & -1.363754 & 1.630142 & 0.260330\end{array}$
C $\quad-2.714371 \quad 2.734146 \quad-0.800507$
N $\quad-3.922897 \quad 3.324158 \quad-0.877350$
H $\quad-4.654953 \quad 3.144669 \quad-0.197754$
$\begin{array}{lllll}\mathrm{H} & -4.200317 & 3.768692 & -1.763474\end{array}$
$\begin{array}{llll}\mathrm{N} & -1.849106 & 2.838089 & -1.812134\end{array}$
H $-0.925110 \quad 2.410248 \quad-1.777557$
$\begin{array}{llll}\mathrm{H} & -2.088898 & 3.286484 & -2.704514\end{array}$
$\begin{array}{llll}\mathrm{N} & 0.075958 & 5.897766 & 1.097870\end{array}$
$\mathrm{H} \quad-0.418121 \quad 6.795364 \quad 1.014633$
$\begin{array}{llll}\text { C } & 0.890396 & 5.504242 & 2.153900\end{array}$
$\begin{array}{llll}\text { C } & 0.076447 & 4.911957 & 0.162979\end{array}$
H $\quad-0.470795 \quad 4.967239 \quad-0.763885$
$\begin{array}{llll}\mathrm{N} & 0.854340 & 3.898422 & 0.573822\end{array}$
C $\quad 1.369110 \quad 4.258820 \quad 1.819489$
H $\quad 2.028305 \quad 3.605392 \quad 2.362061$
$\mathrm{N} \quad 0.184906 \quad-5.580200 \quad-1.435303$
H $\quad 0.410965 \quad-6.315491 \quad-2.153107$
$\begin{array}{llll}\text { C } & -0.451202 & -5.804199 & -0.221268\end{array}$
C $\quad 0.411072-4.261566-1.574585$
$\mathrm{H} \quad 0.916855 \quad-3.802490 \quad-2.400757$
$\begin{array}{llll}\mathrm{N} & -0.065384 & -3.619629 & -0.487228\end{array}$
C $-0.605698 \quad-4.576706 \quad 0.367630$
H $\quad-1.056168 \quad-4.300491 \quad 1.301682$
$\begin{array}{llll}\mathrm{O} & 0.203690 & 0.839813 & 1.384552\end{array}$
$\begin{array}{lllll}\mathrm{O} & 0.348343 & 1.368652 & -0.016251\end{array}$

$\begin{array}{crcc}\mathrm{H} & 0.703217 & -0.022415 & 1.357834 \\ \mathrm{H} & 0.700836 & 2.324478 & 0.179871 \\ \mathrm{Fe} & -0.011414 & -1.759460 & -0.144104 \\ \mathrm{~N} & -0.985214 & -1.033737 & -1.729416 \\ \mathrm{~N} & 1.742996 & -1.519760 & -1.087677 \\ \mathrm{~N} & 1.004059 & -1.916046 & 1.614304 \\ \mathrm{~N} & -1.720047 & -1.710464 & 0.907166 \\ \mathrm{C} & -2.365234 & -0.855698 & -1.876541 \\ \mathrm{C} & -2.682571 & -0.432587 & -3.234848 \\ \mathrm{C} & -1.498444 & -0.334763 & -3.908875 \\ \mathrm{C} & -0.444611 & -0.695404 & -2.972366 \\ \mathrm{C} & 1.928909 & -1.115662 & -2.402786 \\ \mathrm{C} & 3.331192 & -1.219681 & -2.768982 \\ \mathrm{C} & 4.013092 & -1.719086 & -1.693451 \\ \mathrm{C} & 3.020996 & -1.861984 & -0.623627 \\ \mathrm{C} & 2.384766 & -2.063522 & 1.764374 \\ \mathrm{C} & 2.743240 & -2.194855 & 3.166824 \\ \mathrm{C} & 1.578476 & -2.224442 & 3.888016 \\ \mathrm{C} & 0.494003 & -2.096649 & 2.913971 \\ \mathrm{C} & -1.892426 & -2.074997 & 2.241074 \\ \mathrm{C} & -3.301132 & -2.177246 & 2.561157 \\ \mathrm{C} & -3.996674 & -1.813538 & 1.442398 \\ \mathrm{C} & -3.010101 & -1.519028 & 0.412445 \\ \mathrm{C} & -3.289688 & -1.054764 & -0.864487 \\ \mathrm{H} & -4.331223 & -0.862000 & -1.099136 \\ \mathrm{C} & 0.909988 & -0.713089 & -3.254837 \\ \mathrm{H} & 1.205475 & -0.386566 & -4.243820 \\ \mathrm{C} & 3.290161 & -2.086365 & 0.715469 \\ \mathrm{H} & 4.318970 & -2.223587 & 1.002060 \\ \mathrm{C} & -0.863373 & -2.222961 & 3.160080 \\ \mathrm{H} & -1.160446 & -2.482727 & 4.170395 \\ \mathrm{H} & -0.751724 & -6.795364 & 0.118347 \\ \mathrm{H} & -3.685959 & -0.274231 & -3.629868 \\ \mathrm{H} & -1.357407 & -0.070358 & -4.956863 \\ \mathrm{H} & 3.687943 & -0.968246 & -3.767761 \\ \mathrm{H} & 5.077057 & -1.954926 & -1.711426 \\ \mathrm{H} & 3.786652 & -2.156403 & 3.479523 \\ \mathrm{H} & 1.379608 & -2.302109 & 4.956848 \\ \mathrm{H} & -3.677567 & -2.532471 & 3.520401 \\ \mathrm{H} & -5.077042 & -1.743179 & 1.316574 \\ \mathrm{H} & 1.031265 & 6.146362 & 3.023407 \\ \mathrm{H} & -2.845306 & 1.835938 & 1.106232 \\ & & & \end{array}$




\section{TS ; Conformation 2 : Doublet}

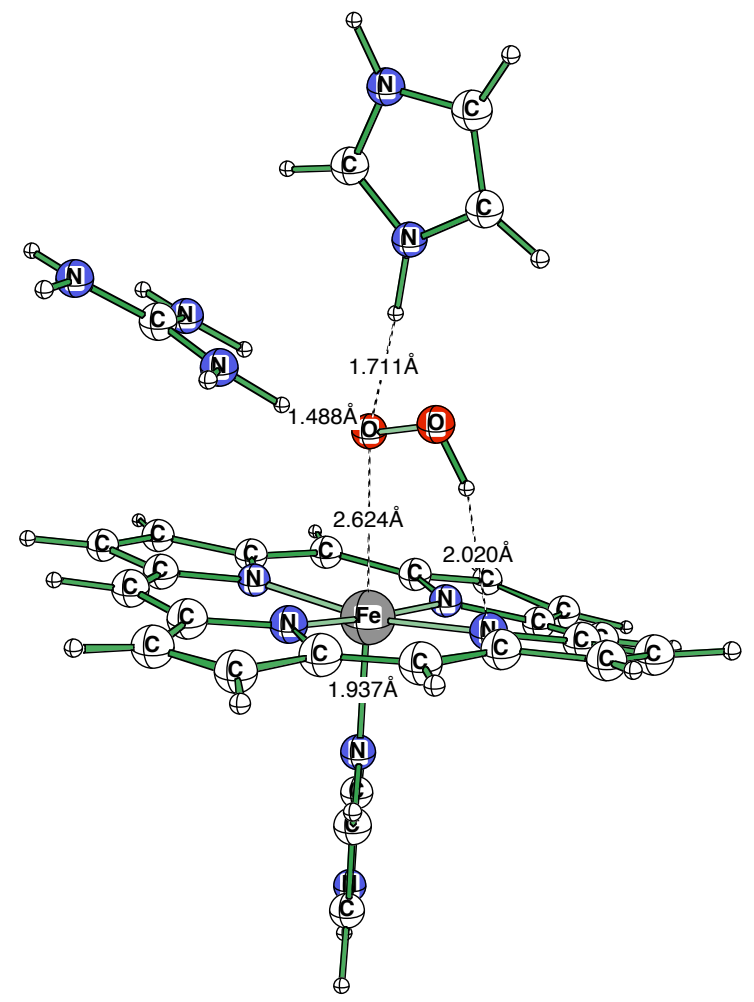

69

$\begin{array}{lrrc}\mathrm{N} & -2.087128 & 1.897995 & 1.454483 \\ \mathrm{H} & -1.243423 & 1.393677 & 0.961441 \\ \mathrm{C} & -2.964523 & 2.553391 & 0.671371 \\ \mathrm{~N} & -4.093719 & 3.139008 & 1.130020 \\ \mathrm{H} & -4.458725 & 2.931564 & 2.053940 \\ \mathrm{H} & -4.743195 & 3.558395 & 0.450912 \\ \mathrm{~N} & -2.637039 & 2.687988 & -0.626999 \\ \mathrm{H} & -1.820480 & 2.220917 & -1.015579 \\ \mathrm{H} & -3.276581 & 3.091293 & -1.320923 \\ \mathrm{~N} & 0.210342 & 5.789246 & 1.127747 \\ \mathrm{H} & -0.233688 & 6.717422 & 1.183578 \\ \mathrm{C} & 1.382675 & 5.404373 & 1.789459 \\ \mathrm{C} & -0.285019 & 4.745407 & 0.434708 \\ \mathrm{H} & -1.179092 & 4.750977 & -0.169876 \\ \mathrm{~N} & 0.536194 & 3.697586 & 0.638458 \\ \mathrm{C} & 1.574722 & 4.080109 & 1.483887 \\ \mathrm{H} & 2.318008 & 3.371338 & 1.793457 \\ \mathrm{~N} & -0.290543 & -5.476135 & -1.208405 \\ \mathrm{H} & -0.374481 & -6.215073 & -1.951920 \\ \mathrm{C} & -0.323761 & -5.711929 & 0.162659 \\ \mathrm{C} & -0.200180 & -4.148300 & -1.422684 \\ \mathrm{H} & -0.134186 & -3.685272 & -2.388062 \\ \mathrm{~N} & -0.173767 & -3.504105 & -0.235504 \\ \mathrm{C} & -0.249222 & -4.479141 & 0.763870 \\ \mathrm{H} & -0.254608 & -4.215668 & 1.804276 \\ \mathrm{O} & 0.891556 & 1.114685 & 1.418793 \\ \mathrm{O} & -0.022919 & 1.029251 & 0.192200 \\ \mathrm{H} & 1.330765 & 0.227798 & 1.372513 \\ \mathrm{H} & 0.353516 & 2.691971 & 0.333389\end{array}$

$\begin{array}{llll}\mathrm{Fe} & -0.044937 & -1.588455 & 0.017578\end{array}$

$\mathrm{N} \quad-1.674866 \quad-1.066391 \quad-1.013153$

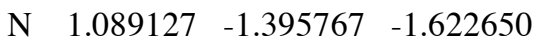

$\begin{array}{llll}\mathrm{N} & 1.642746 & -1.756271 & 1.154175\end{array}$

N $\quad-1.118988-1.646545 \quad 1.716660$

C $\quad-2.976761 \quad-0.955109 \quad-0.529236$

C $\quad-3.884979 \quad-0.588043 \quad-1.610962$

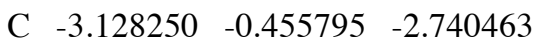

C $\quad-1.749649 \quad-0.739670 \quad-2.364090$

C $\quad 0.656006 \quad-1.034821 \quad-2.889343$

C $\quad 1.755493 \quad-1.128189 \quad-3.837143$

C $\quad 2.855698 \quad-1.586807 \quad-3.165588$

C $2.443665 \quad-1.709259-1.762100$

C $\quad 2.939865 \quad-1.903076 \quad 0.665100$

$\begin{array}{llll}\text { C } & 3.891861 & -2.048721 & 1.758255\end{array}$

$\begin{array}{llll}\text { C } & 3.174149 & -2.089127 & 2.924500\end{array}$

C $\quad 1.766464 \quad-1.956650 \quad 2.539108$

$\begin{array}{llll}\text { C } & -0.671509 & -2.009720 & 2.983932\end{array}$

C $\quad-1.793549 \quad-2.203613 \quad 3.879257$

$\begin{array}{llll}\text { C } & -2.927856 & -1.905502 & 3.176788\end{array}$

C $\quad-2.506760 \quad-1.562195 \quad 1.824127$

$\begin{array}{llll}\text { C } & -3.345078 & -1.162521 & 0.792435\end{array}$

H $\quad-4.394409 \quad-1.037048 \quad 1.038315$

C $-0.653091 \quad-0.699829 \quad-3.211655$

H $\quad-0.838699 \quad-0.393219 \quad-4.233734$

C $3.281860 \quad-1.917557 \quad-0.678345$

H $\quad 4.332245 \quad-2.041962 \quad-0.879776$

C $\quad 0.661545 \quad-2.109802 \quad 3.360779$

H $\quad 0.846863 \quad-2.376724 \quad 4.395798$

H $-0.414841 \quad-6.717422 \quad 0.573517$

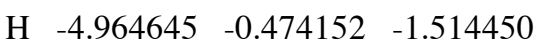

$\mathrm{H} \quad-3.477112 \quad-0.218018 \quad-3.745453$

H $1.622284 \quad-0.913589-4.897568$

H $3.804977 \quad-1.815811 \quad-3.649780$

$\mathrm{H} \quad 4.964645 \quad-2.006470 \quad 1.570206$

H $\quad 3.471970 \quad-2.179230 \quad 3.969238$

H $\quad-1.693909 \quad-2.579788 \quad 4.897552$

$\mathrm{H} \quad-3.955307 \quad-1.899185 \quad 3.540558$

H $\quad 1.912003 \quad 6.105820 \quad 2.434402$

H $\quad-2.208237 \quad 1.703110 \quad 2.427917$ 


\section{Cpd 0 ; Conformation 2 ; Doublet}

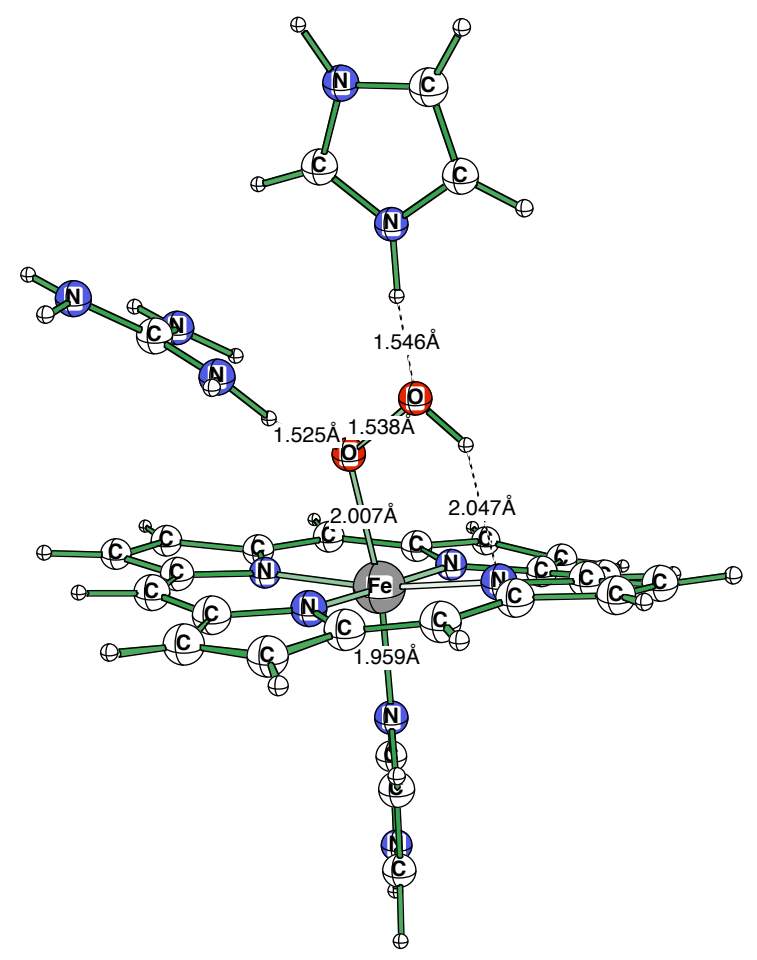

69
$\mathrm{N}-2.319351$
1.566635
H -1.577164
0.988235
1.466507
C -3.261383
2.185486
0.939148
N -4.398285
2.706894
0.723389
$\mathrm{H}-4.727478$
2.439590
1.234177
$\mathrm{H}-5.101883$
$3.085434 \quad 0.583984$
$\mathrm{N}-2.977203$
2.34593
0.583984
H -2.127502
$\begin{array}{ll}1.946320 & -0.976959\end{array}$
$\mathrm{H}-3.657318$
$2.703690-1.260864$
$\mathrm{N}-0.542267$
$\begin{array}{ll}5.817551 & 1.259949\end{array}$
H -1.145645
$\begin{array}{ll}6.652512 & 1.297714\end{array}$
C $\quad 0.724075$
$5.715622 \quad 1.837524$
C -0.848282
$4.656616 \quad 0.638824$
$\mathrm{H}-1.756073$
4.448654
0.093094
$\mathrm{N} \quad 0.195221$
3.820404
0.807602
C 1.179382
$4.461914 \quad 1.545044$
H $\quad 2.099930 \quad 3.974960 \quad 1.794601$
N $\quad 0.276199 \quad-5.397430 \quad-1.208817$
H $0.257629 \quad-6.135498 \quad-1.955002$
C $\quad 0.311157 \quad-5.640656 \quad 0.158875$
C $\quad 0.203690 \quad-4.065414 \quad-1.411270$
H $\quad 0.179642 \quad-3.594818-2.374496$
$\mathrm{N} \quad 0.193115 \quad-3.425827 \quad-0.223190$
$\begin{array}{llll}\text { C } & 0.263992 & -4.408905 & 0.766342\end{array}$
H $\quad 0.262177 \quad-4.152512 \quad 1.808533$
$\begin{array}{llll}\text { O } & 0.540924 & 1.268906 & 0.938309\end{array}$
$\begin{array}{llll}\mathrm{O} & -0.439514 & 0.462723 & 0.069534\end{array}$
$\mathrm{H} \quad 1.271179 \quad 0.602875 \quad 1.045807$
H $\quad 0.276398 \quad 2.764160 \quad 0.650375$
Fe $0.022598 \quad-1.489075 \quad 0.017609$

$\begin{array}{lrrr}\mathrm{N} & -1.692032 & -1.298462 & -1.008514 \\ \mathrm{~N} & 1.097824 & -1.192535 & -1.652573 \\ \mathrm{~N} & 1.760101 & -1.383179 & 1.127960 \\ \mathrm{~N} & -0.990005 & -1.753159 & 1.734009 \\ \mathrm{C} & -2.977875 & -1.312698 & -0.481430 \\ \mathrm{C} & -3.946793 & -1.030685 & -1.537567 \\ \mathrm{C} & -3.238586 & -0.829773 & -2.687836 \\ \mathrm{C} & -1.827225 & -0.972580 & -2.350235 \\ \mathrm{C} & 0.593353 & -0.914734 & -2.911575 \\ \mathrm{C} & 1.683090 & -0.847015 & -3.874557 \\ \mathrm{C} & 2.848190 & -1.136810 & -3.219299 \\ \mathrm{C} & 2.480270 & -1.295593 & -1.806534 \\ \mathrm{C} & 3.054398 & -1.364975 & 0.613693 \\ \mathrm{C} & 4.038712 & -1.400940 & 1.688538 \\ \mathrm{C} & 3.353363 & -1.534195 & 2.866455 \\ \mathrm{C} & 1.929886 & -1.572296 & 2.505737 \\ \mathrm{C} & -0.466324 & -2.022141 & 2.990799 \\ \mathrm{C} & -1.532990 & -2.356079 & 3.914200 \\ \mathrm{C} & -2.712265 & -2.221329 & 3.235184 \\ \mathrm{C} & -2.372192 & -1.849442 & 1.866653 \\ \mathrm{C} & -3.278473 & -1.553665 & 0.853897 \\ \mathrm{H} & -4.327545 & -1.545624 & 1.130524 \\ \mathrm{C} & -0.757980 & -0.785187 & -3.215775 \\ \mathrm{H} & -1.002182 & -0.514175 & -4.236069 \\ \mathrm{C} & 3.363693 & -1.349075 & -0.738159 \\ \mathrm{H} & 4.415512 & -1.322357 & -0.967514 \\ \mathrm{C} & 0.872849 & -1.894089 & 3.344620 \\ \mathrm{H} & 1.114716 & -2.121613 & 4.377396 \\ \mathrm{H} & 0.339157 & -6.652512 & 0.562775 \\ \mathrm{H} & -5.028412 & -1.009384 & -1.405640 \\ \mathrm{H} & -3.627625 & -0.629120 & -3.685913 \\ \mathrm{H} & 1.500015 & -0.655975 & -4.931854 \\ \mathrm{H} & 3.812225 & -1.246597 & -3.715683 \\ \mathrm{H} & 5.101883 & -1.274353 & 1.484894 \\ \mathrm{H} & 3.680984 & -1.608475 & 3.903259 \\ \mathrm{H} & -1.360886 & -2.706161 & 4.931870 \\ \mathrm{H} & -3.723709 & -2.315857 & 3.629990 \\ \mathrm{H} & 1.178482 & 6.493912 & 2.450516 \\ \mathrm{H} & -2.356750 & 1.393890 & 2.450684 \\ & & & \end{array}$

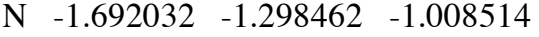

N $1.097824-1.192535 \quad-1.652573$

$\begin{array}{llll}\mathrm{N} & 1.760101 & -1.383179 & 1.127960\end{array}$

$\begin{array}{llll}\mathrm{N} & -0.990005 & -1.753159 & 1.734009\end{array}$

C $-3.946793-1$

$\begin{array}{llll}\text { C } & -3.238586 & -0.829773 & -2.687836\end{array}$

$\begin{array}{llll}\text { C } & -1.827225 & -0.972580 & -2.350235\end{array}$

(

$\begin{array}{llll}\text { C } & 2.848190 & -1.136810 & -3.219299\end{array}$

$\begin{array}{llll}\text { C } & 2.480270 & -1.295593 & -1.806534\end{array}$

$\begin{array}{llll}\text { C } & 3.054398 & -1.364975 & 0.613693\end{array}$

$\begin{array}{llll}\text { C } & 4.038712 & -1.400940 & 1.688538\end{array}$

$\begin{array}{llll}\text { C } & 3.353363 & -1.534195 & 2.866455\end{array}$

$\begin{array}{llll}\text { C } & 1.929886 & -1.572296 & 2.505737\end{array}$

$\begin{array}{llll}\text { C } & -0.466324 & -2.022141 & 2.990799\end{array}$

$\begin{array}{llll}\text { C } & -1.532990 & -2.356079 & 3.914200\end{array}$

$\begin{array}{llll}\text { C } & -2.712265 & -2.221329 & 3.235184\end{array}$

$\begin{array}{llll}\text { C } & -2.372192 & -1.849442 & 1.866653\end{array}$

$\begin{array}{llll}\text { C } & -3.278473 & -1.553665 & 0.853897\end{array}$

$\begin{array}{llll}\text { C } & -4.327545 & -1.545624 & 1.130524\end{array}$

$\begin{array}{llll}\text { C } & -0.757980 & -0.785187 & -3.215775\end{array}$

$\begin{array}{llll}\mathrm{H} & -1.002182 & -0.514175 & -4.236069\end{array}$

$\begin{array}{llll}\text { C } & 3.363693 & -1.349075 & -0.738159\end{array}$

$\begin{array}{llll}\text { C } & 4.415512 & -1.322357 & -0.967514\end{array}$

$\begin{array}{llll}\text { C } & 0.872849 & -1.894089 & 3.344620\end{array}$

$\begin{array}{llll}\mathrm{H} & 1.114716 & -2.121613 & 4.377396\end{array}$

$\mathrm{H} \quad 0.339157 \quad-6.652512 \quad 0.562775$

$\begin{array}{llll}\mathrm{H} & -5.028412 & -1.009384 & -1.405640\end{array}$

$\mathrm{H}-3.627625-0.629120-3.685913$

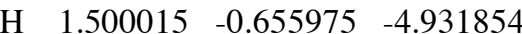

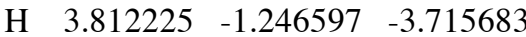

H $\quad 5.101883 \quad-1.274353 \quad 1.484894$

H $3.680984 \quad-1.608475 \quad 3.903259$

$\begin{array}{llll}\mathrm{H} & -1.360886 & -2.706161 & 4.931870\end{array}$

$\mathrm{H} \quad 1.178482 \quad 6.493912 \quad 2.450516$

$\begin{array}{llll}\mathrm{H} & -2.356750 & 1.393890 & 2.450684\end{array}$ 


\section{Complex with $\mathrm{H}_{2} \mathrm{O}_{2}$ Quartet}

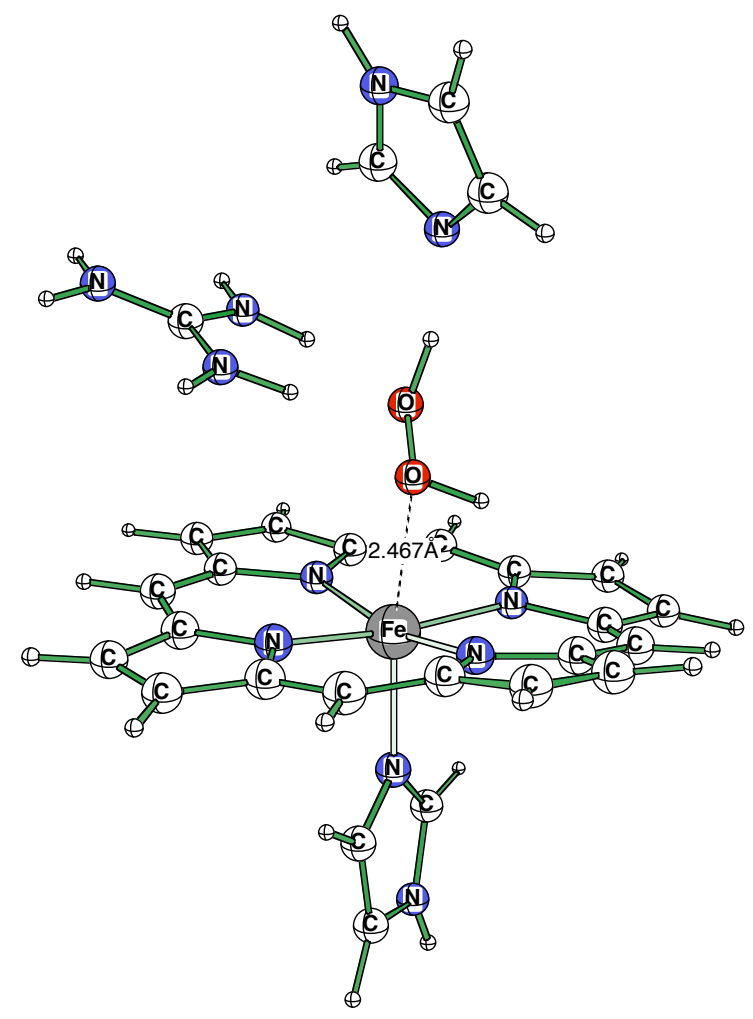

69

N $\quad-4.407000-0.436000-2.868000$

$\mathrm{H} \quad-3.644000-1.132000-2.982000$

C $\quad-5.311000-0.364000-3.872000$

N $\quad-6.3530000 .488000-3.860000$

H $\quad-6.6310000 .997000-3.025000$

H $\quad-7.0290000 .436000-4.634000$

N $\quad-5.090000-1.152000-4.931000$

H $-4.327000-1.828000-4.945000$

H $\quad-5.736000-1.209000-5.727000$

N $\quad-1.7490001 .988000-5.612000$

H $\quad-2.1810002 .781000-6.105000$

C $\quad-0.5010001 .994000-5.002000$

C $\quad-2.2660000 .735000-5.526000$

$\mathrm{H} \quad-3.2210000 .449000-5.937000$

N $\quad-1.400000-0.066000-4.882000$

C $\quad-0.2880000 .712000-4.562000$

H $\quad 0.5730000 .300000-4.071000$

$\mathrm{N} \quad-3.548000-8.0990000 .350000$

H $\quad-3.755000-9.1240000 .286000$

C $\quad-3.546000-7.3420001 .509000$

C $-3.322000-7.279000-0.699000$

$\mathrm{H} \quad-3.229000-7.600000-1.719000$

N $-3.184000-6.016000-0.259000$

C $\quad-3.325000-6.0450001 .123000$

H $\quad-3.260000-5.1460001 .706000$

O $-1.852000-2.158000-2.113000$

O $\quad-2.366000-2.039000-3.518000$

H $\quad-1.076000-2.770000-2.178000$
H $\quad-1.815000-1.286000-4.008000$

FE $-2.797000-4.278000-1.276000$

N $-4.413000-4.250000-2.490000$

$\mathrm{N} \quad-1.719000-5.234000-2.690000$

$\mathrm{N}-1.033000-3.882000-0.286000$

N $\quad-3.767000-3.0990000 .008000$

C $\quad-5.670000-3.705000-2.227000$

C $-6.601000-4.044000-3.298000$

C $-5.903000-4.784000-4.211000$

C $-4.537000-4.898000-3.716000$

C $\quad-2.219000-5.764000-3.874000$

C $-1.216000-6.600000-4.507000$

C $\quad-0.107000-6.617000-3.702000$

C $\quad-0.404000-5.721000-2.585000$

C $\quad 0.234000-4.352000-0.614000$

C $\quad 1.231000-3.8020000 .293000$

C $\quad 0.568000-3.0440001 .221000$

C $-0.856000-3.1220000 .867000$

C $-3.265000-2.6020001 .210000$

C $-4.333000-1.9810001 .967000$

C $\quad-5.466000-2.0390001 .203000$

C $-5.115000-2.740000-0.021000$

C $-5.972000-2.971000-1.090000$

H $\quad-6.979000-2.577000-1.005000$

C $-3.509000-5.571000-4.353000$

H $\quad-3.737000-6.007000-5.319000$

C $\quad 0.498000-5.243000-1.645000$

H $\quad 1.527000-5.545000-1.748000$

C $\quad-1.927000-2.6010001 .584000$

H $\quad-1.701000-2.1430002 .540000$

h $\quad-3.690703-7.7590422 .505596$

h $-7.660344-3.788648-3.323535$

h $-6.279796-5.254437-5.119174$

h $-1.426822-7.140091-5.429997$

h $0.772795-7.230500-3.896026$

h $2.292303-3.9780170 .117725$

h $0.914645-2.4542332 .069574$

h $\quad-4.212382-1.5929252 .978393$

h $-6.456024-1.6410921 .425682$

h $0.1047542 .897151-4.928199$

h $-4.4511240 .009190-1.973668$ 


\section{TS ; Quartet}

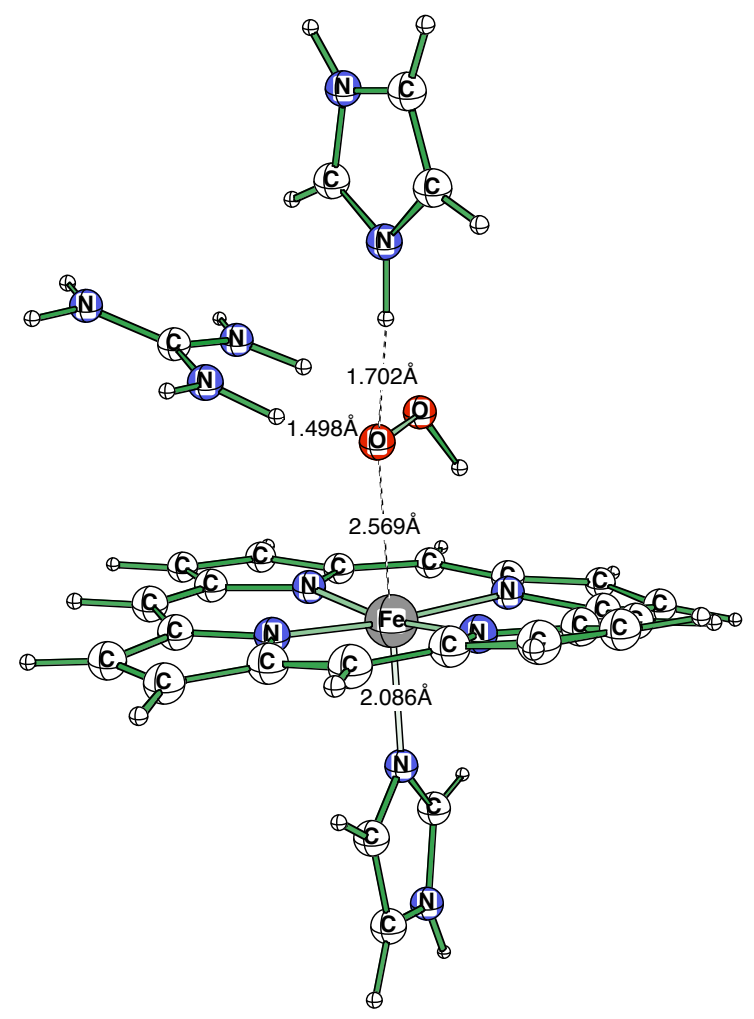

69
$\begin{array}{llll}\mathrm{N} & -4.413621 & -0.540953 & -2.644651\end{array}$
H $\quad-3.588120 \quad-1.251864 \quad-2.508387$
C $\quad-5.219320 \quad-0.526271 \quad-3.725116$
$\begin{array}{llll}\mathrm{N} & -6.261524 & 0.326575 & -3.861587\end{array}$
$\mathrm{H} \quad-6.637662 \quad 0.821377 \quad-3.060970$
$\mathrm{H} \quad-6.872720 \quad 0.259983 \quad-4.687886$
N $-4.877470 \quad-1.334737 \quad-4.736973$
H $\quad-4.070171 \quad-1.965642 \quad-4.656043$
H $\quad-5.466374 \quad-1.456919 \quad-5.571297$
$\begin{array}{llll}\mathrm{N} & -1.827195 & 1.931043 & -5.340655\end{array}$
H $\quad-2.213403 \quad 2.604518 \quad-6.013398$
C $\quad-0.619651 \quad 2.077580 \quad-4.656391$
$\begin{array}{llll}\text { C } & -2.421836 & 0.779103 & -4.949937\end{array}$

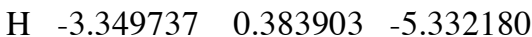
$\begin{array}{llll}\mathrm{N} & -1.627838 & 0.203330 & -4.025169\end{array}$
$\begin{array}{llll}\text { C } & -0.508007 & 0.989831 & -3.833618\end{array}$
$\mathrm{H} \quad 0.246432 \quad 0.706483 \quad-3.129861$
$\begin{array}{llll}\mathrm{N} & -3.545439 & -8.035090 & 0.319257\end{array}$
$\begin{array}{llll}\mathrm{H} & -3.749091 & -9.060807 & 0.249168\end{array}$
C $\quad-3.538966 \quad-7.292366 \quad 1.488018$
C $-3.322660 \quad-7.199868-0.721064$
$\mathrm{H} \quad-3.247203 \quad-7.508198 \quad-1.746182$
N $-3.180926 \quad-5.942171 \quad-0.266717$
C $\quad-3.315684-5.991202 \quad 1.115837$
H $\quad-3.246520 \quad-5.100049 \quad 1.711099$
$\begin{array}{llll}\mathrm{O} & -2.239014 & -1.871795 & -2.308513\end{array}$
O $-2.202710 \quad-2.485282 \quad-3.694677$
H $\quad-1.829909 \quad-0.638535 \quad-3.407168$

$\begin{array}{rrrr}\mathrm{H} & -1.947677 & -3.427949 & -3.524247 \\ \mathrm{Fe} & -2.787134 & -4.156369 & -1.270030 \\ \mathrm{~N} & -4.436595 & -4.218829 & -2.465432 \\ \mathrm{~N} & -1.732001 & -5.146546 & -2.711506 \\ \mathrm{~N} & -1.071333 & -3.840700 & -0.245056 \\ \mathrm{~N} & -3.780396 & -3.028165 & 0.034583 \\ \mathrm{C} & -5.694824 & -3.682208 & -2.195858 \\ \mathrm{C} & -6.633970 & -4.040698 & -3.253334 \\ \mathrm{C} & -5.942045 & -4.793003 & -4.160541 \\ \mathrm{C} & -4.573106 & -4.891995 & -3.676879 \\ \mathrm{C} & -2.255692 & -5.755895 & -3.858914 \\ \mathrm{C} & -1.258411 & -6.614174 & -4.460151 \\ \mathrm{C} & -0.142930 & -6.599776 & -3.661101 \\ \mathrm{C} & -0.426472 & -5.660182 & -2.579558 \\ \mathrm{C} & 0.184726 & -4.308058 & -0.584627 \\ \mathrm{C} & 1.194532 & -3.764277 & 0.320001 \\ \mathrm{C} & 0.539981 & -3.003863 & 1.250315 \\ \mathrm{C} & -0.888553 & -3.075054 & 0.897751 \\ \mathrm{C} & -3.292657 & -2.553865 & 1.248043 \\ \mathrm{C} & -4.374397 & -1.975606 & 2.017735 \\ \mathrm{C} & -5.502326 & -2.030005 & 1.244789 \\ \mathrm{C} & -5.136825 & -2.701746 & 0.008138 \\ \mathrm{C} & -5.993666 & -2.946384 & -1.058298 \\ \mathrm{H} & -7.005723 & -2.567230 & -0.966623 \\ \mathrm{C} & -3.554106 & -5.581488 & -4.318068 \\ \mathrm{H} & -3.804980 & -6.052525 & -5.261024 \\ \mathrm{C} & 0.456937 & -5.196207 & -1.617473 \\ \mathrm{H} & 1.484938 & -5.508197 & -1.698625 \\ \mathrm{C} & -1.951700 & -2.544027 & 1.616476 \\ \mathrm{H} & -1.718742 & -2.081045 & 2.568666 \\ \mathrm{H} & -3.692690 & -7.722528 & 2.477662 \\ \mathrm{H} & -7.691686 & -3.779465 & -3.285885 \\ \mathrm{H} & -6.313347 & -5.276432 & -5.064143 \\ \mathrm{H} & -1.475746 & -7.181413 & -5.365176 \\ \mathrm{H} & 0.730721 & -7.225069 & -3.844972 \\ \mathrm{H} & 2.255498 & -3.928955 & 0.132122 \\ \mathrm{H} & 0.892691 & -2.420469 & 2.100794 \\ \mathrm{H} & -4.277702 & -1.639123 & 3.049964 \\ \mathrm{H} & -6.494609 & -1.636211 & 1.464716 \\ \mathrm{H} & 0.029954 & 2.947030 & -4.757069 \\ \mathrm{H} & -4.506457 & 0.005588 & -1.812397\end{array}$

H $\quad-1.947677 \quad-3.427949 \quad-3.524247$

$\mathrm{N} \quad-1.732001 \quad-5.146546 \quad-2.711506$

$\mathrm{N}-1.071333 \quad-3.840700 \quad-0.245056$

$\begin{array}{llll}\mathrm{N} & -3.780396 & -3.028165 & 0.034583\end{array}$

$\begin{array}{llll}\text { C } & -5.694824 & -3.682208 & -2.195858\end{array}$

C $\quad-6.633970 \quad-4.040698 \quad-3.253334$

C $-4.573106-4.891995 \quad-3.676879$

$\begin{array}{llll}\text { C } & -2.255692 & -5.755895 & -3.858914\end{array}$

C $\quad-1.258411 \quad-6.614174 \quad-4.460151$

C $-0.426472-5.660182-2579558$

$\begin{array}{llll}\text { C } & 0.184726 & -4.308058 & -0.584627\end{array}$

$\begin{array}{llll}\text { C } & 1.194532 & -3.764277 & 0.320001\end{array}$

$\begin{array}{llll}\text { C } & 0.539981 & -3.003863 & 1.250315\end{array}$

$\begin{array}{llll}\text { C } & -0.888553 & -3.075054 & 0.897751\end{array}$

C $\quad-4.374397-1.975606 \quad 2.017735$

$\begin{array}{llll}\text { C } & -5.502326 & -2.030005 & 1.244789\end{array}$

$\begin{array}{llll}\text { C } & -5.136825 & -2.701746 & 0.008138\end{array}$

C $\quad-5.993666-2.946384-1.058298$

H $\quad-7.005723 \quad-2.567230 \quad-0.966623$

C $-3.554106-5.581488-4.318068$

$\begin{array}{llll}\text { H } & -3.804980 & -6.052525 & -5.261024\end{array}$

C $\quad 0.456937 \quad-5.196207 \quad-1.617473$

H $1.484938 \quad-5.508197 \quad-1.698625$

C $\quad-1.951700 \quad-2.544027 \quad 1.616476$

H $-1.718742-2.081045-2.568666$

$\begin{array}{lll}-3.692690 & -7.722528 & 2.477662\end{array}$

H $\quad-6.313347 \quad-5.276432 \quad-5.064143$

$\mathrm{H} \quad-1.475746 \quad-7.181413 \quad-5.365176$

H $\quad 0.730721 \quad-7.225069 \quad-3.844972$

H $2.255498 \quad-3.928955 \quad 0.132122$

H $\quad 0.892691 \quad-2.420469 \quad 2.100794$

$\mathrm{H}-4.277702-1.639123 \quad 3.049964$

H $\quad 0.029954 \quad 2.947030 \quad-4.757069$ 


\section{Cpd 0 Quartet}

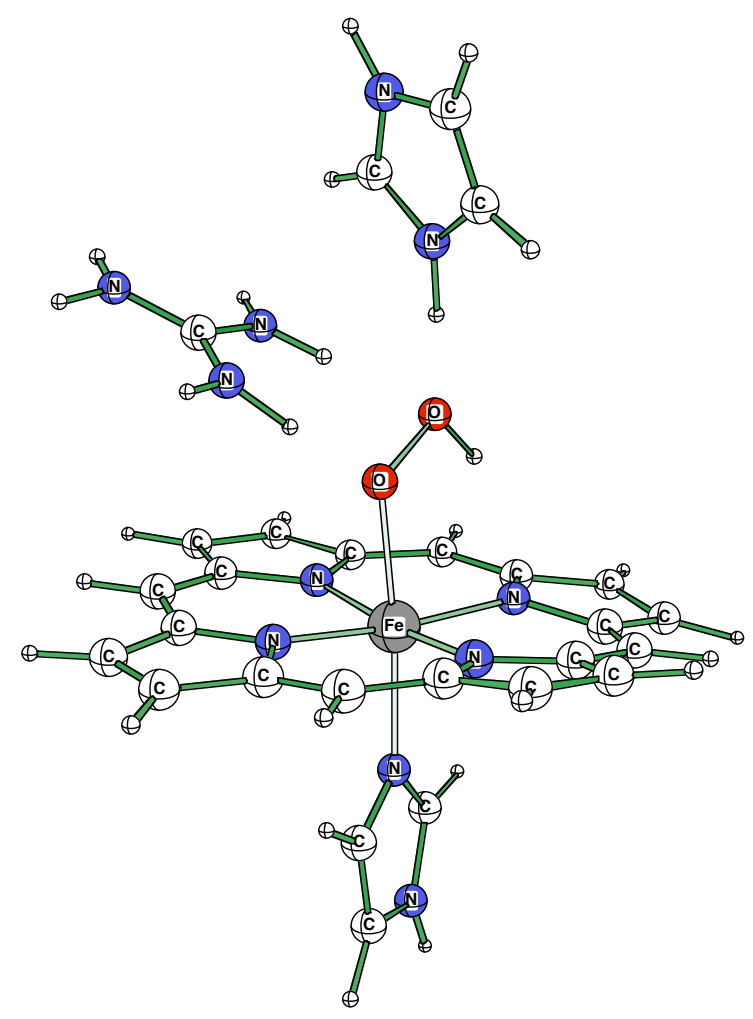

69

$\mathrm{N} \quad-4.399000-0.544000-2.681000$

H $\quad-3.667000-1.335000-2.497000$

C $\quad-5.220000-0.491000-3.748000$

$\mathrm{N} \quad-6.2500000 .381000-3.856000$

H $\quad-6.6090000 .869000-3.041000$

$\mathrm{H} \quad-6.8740000 .327000-4.673000$

N $\quad-4.925000-1.295000-4.780000$

H $\quad-4.136000-1.940000-4.729000$

H $\quad-5.546000-1.412000-5.591000$

$\mathrm{N} \quad-1.8170001 .990000-5.506000$

$\mathrm{H} \quad-2.2190002 .753000-6.071000$

C $\quad-0.5850002 .031000-4.853000$

C $\quad-2.3870000 .783000-5.314000$

$\mathrm{H} \quad-3.3370000 .458000-5.711000$

$\mathrm{N} \quad-1.5480000 .055000-4.553000$

C $\quad-0.4210000 .810000-4.257000$

H $\quad 0.3800000 .416000-3.662000$

$\mathrm{N} \quad-3.538000-8.0540000 .317000$

H $\quad-3.743000-9.0780000 .253000$

C $\quad-3.531000-7.3020001 .480000$

C $-3.315000-7.224000-0.730000$

H $\quad-3.238000-7.540000-1.752000$

N $-3.171000-5.963000-0.288000$

C $\quad-3.305000-6.0040001 .096000$

H $\quad-3.234000-5.1100001 .686000$

O $-2.465000-2.156000-2.138000$

O $-2.110000-2.263000-3.610000$

H $\quad-1.732000-0.927000-4.151000$
H $-1.893000-3.221000-3.720000$

FE $-2.786000-4.156000-1.314000$

N $\quad-4.455000-4.258000-2.493000$

N $\quad-1.724000-5.125000-2.765000$

$\mathrm{N} \quad-1.073000-3.873000-0.251000$

N $\quad-3.787000-3.0430000 .020000$

C $\quad-5.706000-3.715000-2.216000$

C $-6.648000-4.066000-3.276000$

C $-5.960000-4.814000-4.189000$

C $-4.587000-4.914000-3.711000$

C $\quad-2.246000-5.725000-3.915000$

C $-1.247000-6.586000-4.516000$

C $-0.138000-6.585000-3.708000$

C $\quad-0.426000-5.651000-2.621000$

C $\quad 0.181000-4.338000-0.593000$

C $\quad 1.193000-3.7950000 .314000$

C $\quad 0.538000-3.0340001 .243000$

C $-0.892000-3.1060000 .889000$

C $-3.295000-2.5590001 .225000$

C $-4.373000-1.9600001 .985000$

C $\quad-5.504000-2.0270001 .217000$

C $-5.141000-2.715000-0.013000$

C $-5.997000-2.971000-1.079000$

H $\quad-7.009000-2.589000-0.989000$

C $-3.553000-5.569000-4.367000$

H $\quad-3.797000-6.034000-5.315000$

C $\quad 0.452000-5.212000-1.640000$

H $\quad 1.478000-5.530000-1.718000$

C $-1.955000-2.5620001 .600000$

H $-1.725000-2.0980002 .553000$

h $\quad-3.686947-7.7262352 .471853$

h $-7.703213-3.794701-3.307444$

h $-6.341266-5.296343-5.089027$

h $-1.465962-7.158727-5.417169$

h $0.731666-7.217413-3.886333$

h $2.253871-3.9580970 .124215$

h $0.889335-2.4458282 .090752$

h $-4.262007-1.5940223 .005689$

h $\quad-6.496599-1.6347811 .438308$

h $0.0450822 .920398-4.847173$

h $-4.4684610 .005071-1.848132$ 


\section{Reference 7b in Full}

Sherwood P.; de Vries A.H.; Guest M.F.; Schreckenbach G.; Catlow C.R.A.; French S.A.; Sokol A.A.; Bromley S.T.; Thiel W.; Turner A.J.; Billeter S.; Terstegen F.; Thiel S.; Kendrick J.; Rogers S.C.; Casci J.; Watson M.; King F.; Karlsen E.; Sjovoll M.; Fahmi A.; Schafer A.; Lennartz C. J. Mol. Struc. (Theochem) 2003, 632, 1-28 


\section{CPMD and QM/MM calculations on the conversion of Conf 2 to Conf 3 and classical MD simulations on the departure of W2407}

\section{CPMD Calculations}

\section{Computational details}

$\mathrm{Ab}$ initio molecular dynamics simulations were performed for Cpd 0 , using the method developed by Laio, VandeVondele, and Röthlisberger. ${ }^{1}$ This method combines the first principles molecular dynamics method of Car and Parrinello (CPMD) ${ }^{2}$ with a force-field molecular dynamics methodology. Previous work has demonstrated the reliability of this method in the description of structural, energetic, and dynamic properties of systems of biological interest, including hemeproteins. ${ }^{3}$ The starting structure of $\mathrm{Cpd} 0$ was built from the HRP: $\mathrm{H}_{2} \mathrm{O}_{2}$ model described in Section $\mathrm{C}$, by adjusting the coordinates of $\mathrm{H}_{2} \mathrm{O}_{2}$, WAT2407 and His42 to the Conf.2 of Cpd 0 (Figure 2 text). The simulations were performed within a fully Hamiltonian-coupling scheme between the DFT region (the active site) and the classical regions (the rest of the protein in aqueous solution, in the presence of counterions). The QMMM partition was similar to the one used in the static QM/MM calculations, including the vinyl and methyl substituents of the heme group within the QM region. Dangling bonds were saturated by hydrogen atoms. Spurious electrostatic interactions between the capping hydrogens and close-by classical atoms were excluded from the QM/MM Hamiltonian according to reference 3. Valence orbitals were expanded with a plane wave basis set up to 70 Ry. Martins-Troullier pseudopotentials were used to describe the core electron-valence shell electron interactions. ${ }^{4}$ The Becke and Perdew functionals were used for exchange and correlation interactions, respectively. ${ }^{5}$ Electrostatic interactions were taken into account within a multilayer approach. The $\mathrm{QM} / \mathrm{MM}$ electrostatic interactions were evaluated as follows: (i) a modified Coulomb interaction between the QM electronic density and the MM atoms within $7.9 \AA$ from any QM atom. (ii) Coulomb interactions between D-RESP point charges centered on the QM atoms and RESP charges on the MM atoms between 7.9 $\AA$ and $26.5 \AA$ from any QM atom. (iii) All protein and solvent atoms were allowed to move, except the peroxide $\mathrm{O}-\mathrm{O}$ distance that was kept fixed. Electrostatic interactions between a multipole expansion representing the charge distribution of the $\mathrm{QM}$ region and the $\mathrm{MM}$ atoms beyond 26.5 $\AA$ from any QM atom. Bonded and van der Waals interactions between the QM and MM parts, as well as within the MM region, were accounted for by the AMBER force field. The QM region was placed in a supercell and treated as isolated. A CPMD simulation for $1.20 \mathrm{ps}$ was performed at $300 \mathrm{~K}$ coupling the systems to a Nose-Hoover thermostat, using a time step of 5 a.u. and a fictitious electron mass of 800 a.u. to solve the equations of motion.

\section{Results}

The simulation shows that His42 is very mobile, it rotates around $C_{\beta}-C_{\gamma}$ and changes hydrogen bond partner easily. At the beginning of the simulation the shortest hydrogen bond distance involves the oxygen atom of W2407 (Figure S6). However, after about 0.25 ps His42- $\mathrm{H}^{+}$switches partner to the distal oxygen atom of the peroxide, forming Conf.3. Sometime later (at $\approx 0.75 \mathrm{ps}$ ), the system goes back to Conf.2 (Figure S6). This indicates that there are two local minima (Conf.2 and Conf.3) with similar energy and separated by a low energy barrier. In Conf.2, formation of Cpd I is hampered by the presence of W2407. In 
contrast, in the new conformation (Conf.3) the water molecule is displaced and can easily escape to the solvent, since it is already part of the solvent network (Fig. S2). In the next section we will demonstrate that at longer timescales W2407 indeed escapes through the channel. Interestingly, Conf.3 is very similar to the one previously found in the absence of $\mathrm{W} 2407,{ }^{6}$ except for a $90^{\circ}$ rotation of the $\mathrm{OOH}$ ligand with respect to the $\mathrm{Fe}-\mathrm{O}_{1}$ bond.

Therefore, the simulation highlights the dynamics of hydrogen bonds in the HRP pocket and shows that W2407 switches partners easily, leading to a new conformation (conf.3) in which the protonated histidine (His- $\mathrm{H}^{+} 42$ ) forms a hydrogen bond with the distal oxygen of Cpd 0 , in a manner very similar to what was found in reference 6 (ref 3 in the paper Text) to lead to a facile $\mathrm{O}-\mathrm{O}$ cleavage and formation of Cpd I.
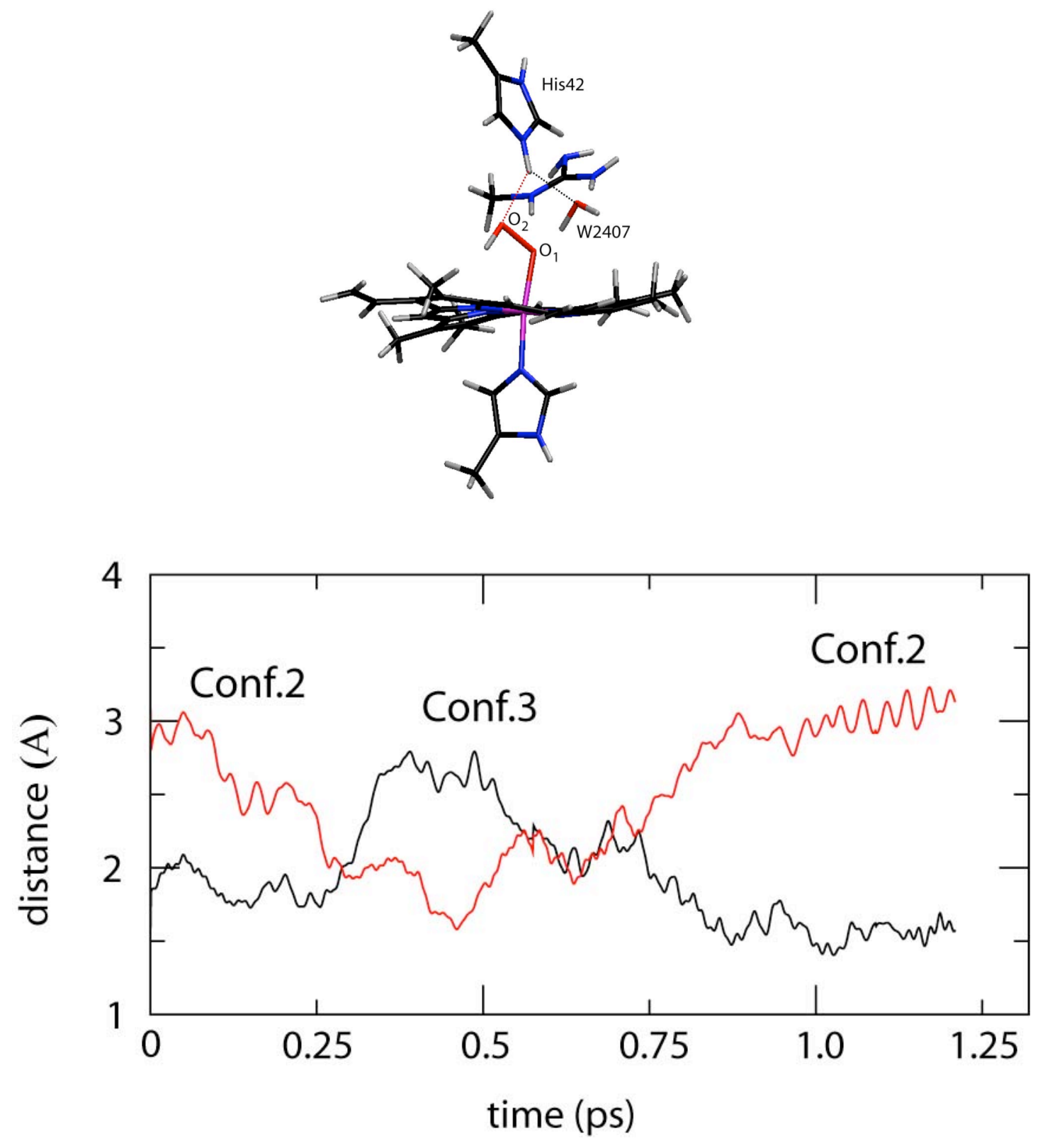

Figure S6. Time evolution of the $\mathrm{H}_{\mathrm{His}} \cdots \mathrm{O}_{\mathrm{W} 2407}$ (black line) and $\mathrm{H}_{\mathrm{Hi}} \cdots \mathrm{O}_{2}$ (red line) distances during the room temperature CPMD simulation of Cpd 0. Conf.2 corresponds to the situations 
in which the red line is above the black line (i.e. $\mathrm{H}_{\mathrm{His}} \cdots \mathrm{O}_{2}>\mathrm{H}_{\mathrm{His}} \cdots \mathrm{O}_{\mathrm{W} 2407}$ ). Converserly, Conf.3 occurs when the red line is below the black line (i.e. $\mathrm{H}_{\mathrm{His}} \cdots \mathrm{O}_{2}<\mathrm{H}_{\mathrm{His}} \cdots \mathrm{O}_{\mathrm{w} 2407}$ ).

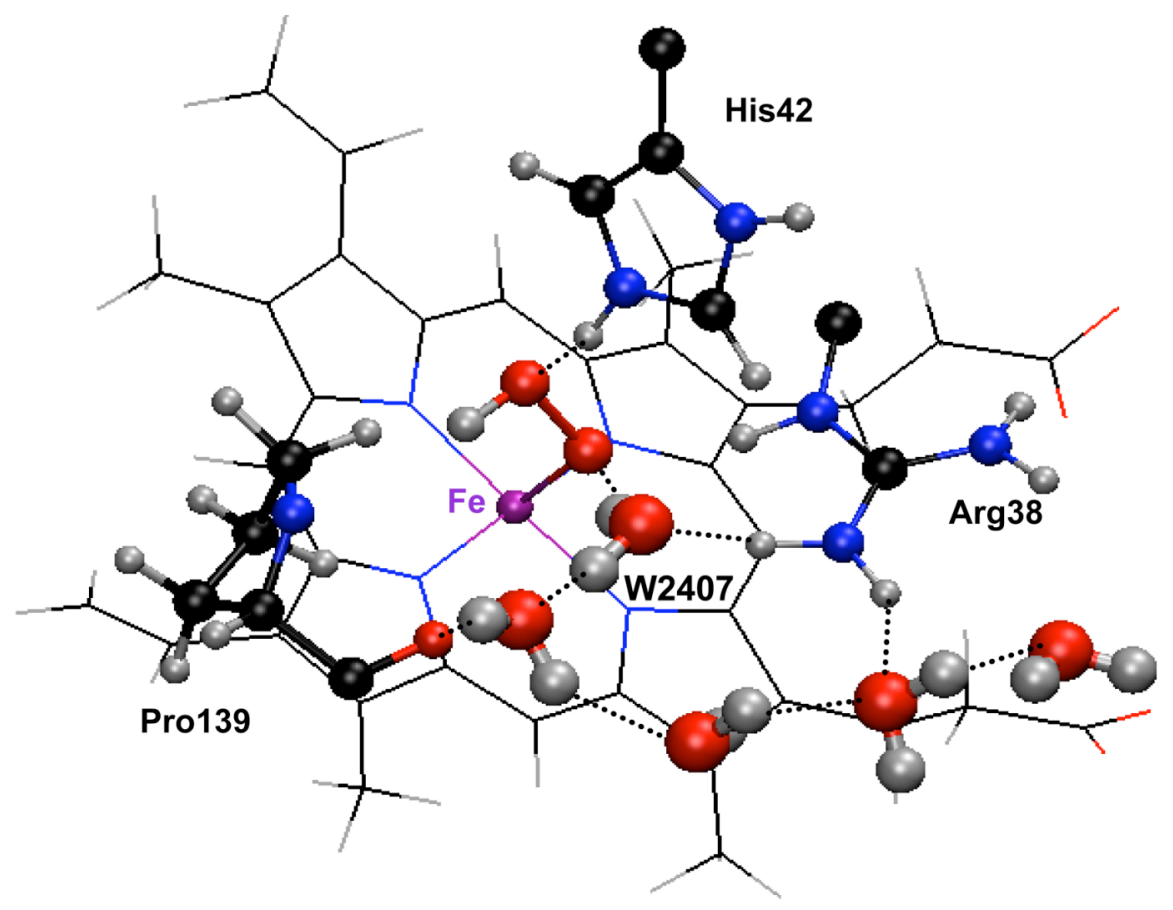

Figure S7. Water network in Conf. 3 of Cpd 0, obtained from the CPMD simulation.

${ }^{1}$ Laio, A.; vandeVondele, J.; Röthlisberger, U. J. Chem. Phys. 2002, 116, 6941-6947.

${ }^{2}$ (a) Car, R.; Parrinello, M. Phys. Rev. Lett. 1985, 55, 2471-2474. (b) Marx, D.; Hutter, J. $2000 \mathrm{Ab}$ initio molecular dynamics: Theory and implementation, in Modern methods and algorithms of Quantum Chemistry (Grotendorst, J., ed.), John von Neumann Institute for Computing, Julich, Germany, pp. 301-409. (c) CPMD program, Copyright IBM Corp. 19902003, Copyright MPI für Festkörperforschung, Stuttgart 1997-2001. URL:

http://www.cpmd.org.

${ }^{3}$ See for instance: (a) Carloni, P.; Röthlisberger, U.; Parrinello, M. Acc. Chem. Res. 2002, 35, 455-464. (b) Blumberger, J.; Klein, M. L. J. Am. Chem. Soc. 2006, 128, 13854-13867. (c) Alfonso-Prieto, M.; Borovik, A.; Carpena, X.; Murshudov, G.; Melik-Adamyan, W.; Fita, I.; Rovira, C.; Loewen, P. C. 2007 In press.

${ }^{3}$ Laio, A., Gervasio, F. L., VandeVondele, J., Sulpizi, M., and Rothlisberger, U. (2004) J. Phys. Chem. B 108, 7963-7968.

${ }^{4}$ Troullier, M. ; Martins, J. L. Phys. Rev. B 1991, 43, 1993-2006.

5 (a) A. D. Becke, J. Chem. Phys. 1986, 84, 4524-4529. (b) J. P. Perdew, Phys. Rev. B. 1986, 33, 8822-8824.

${ }^{6}$ Derat, E.; Shaik, S. J. Phys. Chem. B 2006, 110, 10526-10533. 


\section{Chemshell QM/MM Calculations on the Conf.2 and Conf. 3 Structures Obtained from CPMD}

Two snapshots were selected from the CPMD simulation, at 0.5 and $1 \mathrm{ps,} \mathrm{corresponding} \mathrm{to}$ Conf. 3 and Conf. 2 of Compound 0, respectively. These snapshots were used for subsequent ChemShell calculations. Since CPMD simulations use periodic conditions and the Chemshell $\mathrm{QM} / \mathrm{MM}$ implementation uses a shell of solvation waters, the enzyme plus a layer of $15 \AA$ water was extracted from the cubic box used by CPMD. This system was then readapted to be suitable for Chemshell calculations (modifications of the PDB format, generation of the PSF file). The level of calculations used is the same than for the previously described structures

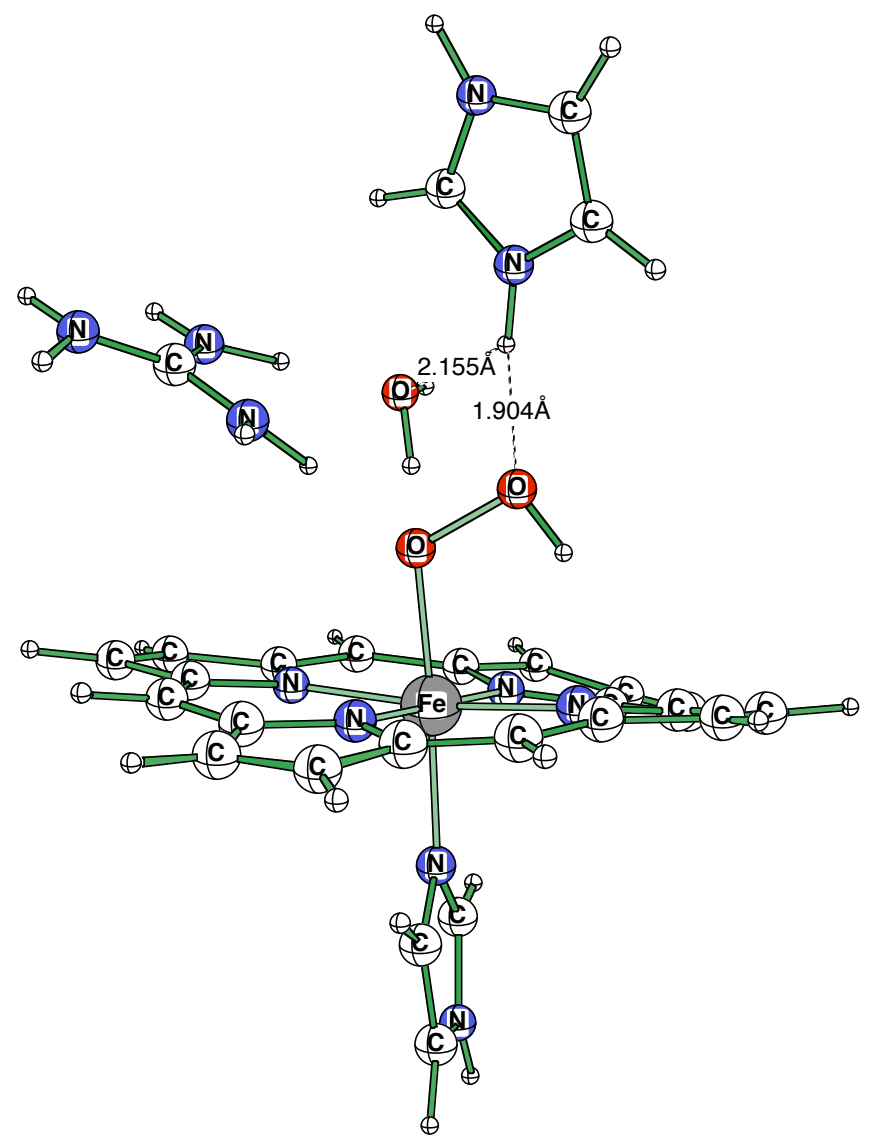

Figure S8: Chemshell QM/MM optimized structure (B3LYP/B1) of a representative snapshot of the CPMD simulation corresponding to Conf. 3 (see Figure S6). The hydrogen bond between His 42 and $\mathrm{OH}$ of $\mathrm{FeOOH}$ observed during CPMD is maintained during the B3LYP optimization. Therefore Conf. 3 of Compound 0 is a stable conformation.

Since during the CPMD simulations, all the atoms in the box are allowed to move and rearrange, it is difficult to compare two conformations by reoptimizing it with Chemshell (only a small part around the active site is reoptimized). Therefore, to evaluate relative energies, we chose to do only single point energy calculations with Chemshell on the two snapshots extracted from CPMD and to compare only the QM energies. The first snapshot corresponds to Conf. 2 and has a QM energy of -1997.737223615, the second to Conf. 3 and has a QM energy of -1997.745681703 . The difference is thus $5.31 \mathrm{kcal} / \mathrm{mol}$ in favor of Conf. 3. 


\section{Classical MD simulations of the HRP - OOH complex. The escape of W2407 from the binding pocket.}

Classical MD simulations of Cpd 0 were performed in order to extend the sampling of the configuration space. The calculations were based on the last frame from the CPMD simulation of Cpd 0 (corresponding to Conf.2). The force field parameters for the heme$\mathrm{O}_{1} \mathrm{O}_{2} \mathrm{H}_{2}$ moiety were adapted from those of the AMBER distribution, introducing the following harmonic terms:

$\begin{array}{lll}\text { bond } & \text { equil. value } & \text { force constant } \\ \mathrm{Fe}-\mathrm{O}_{1} & 1.95 \AA & 100.0 \mathrm{kcal} \mathrm{mol}^{-1} \AA^{-2} \\ \mathrm{O}_{1}-\mathrm{O}_{2} & 1.52 \AA & 100.0 \mathrm{kcal} \mathrm{mol}^{-1} \AA^{-2} \\ & & \\ \text { angle } & & \\ \mathrm{Fe}-\mathrm{O}_{1}-\mathrm{O}_{2} & 118.0^{\circ} & 70.0 \mathrm{kcal} \mathrm{mol}^{-1} \mathrm{deg}^{-2} \\ \mathrm{O}_{1}-\mathrm{O}_{2}-\mathrm{H}_{2} & 100.0^{\circ} & 50.0 \mathrm{kcal} \mathrm{mol}^{-1} \mathrm{deg}^{-2} \\ \mathrm{~N}_{\text {porph }}-\mathrm{Fe}-\mathrm{O}_{1} & 90.0^{\circ} & 50.0 \mathrm{kcal} \mathrm{mol}^{-1} \mathrm{deg}^{-2}\end{array}$

The equilibrium bond lengths and angles were set at the average values from the CPMD simulation of Cpd 0. Charges on the Fe, $\mathrm{O}_{1}, \mathrm{O}_{2}, \mathrm{H}_{2}$ atoms were set to $0.75,-0.5,-0.5,0.5$ a.u. These parameters turned out to reproduce fairly well the conformational properties of $\mathrm{Cpd} 0$ compared to the CPMD simulation. Computational details of the classical MD simulations are as reported in Section C.
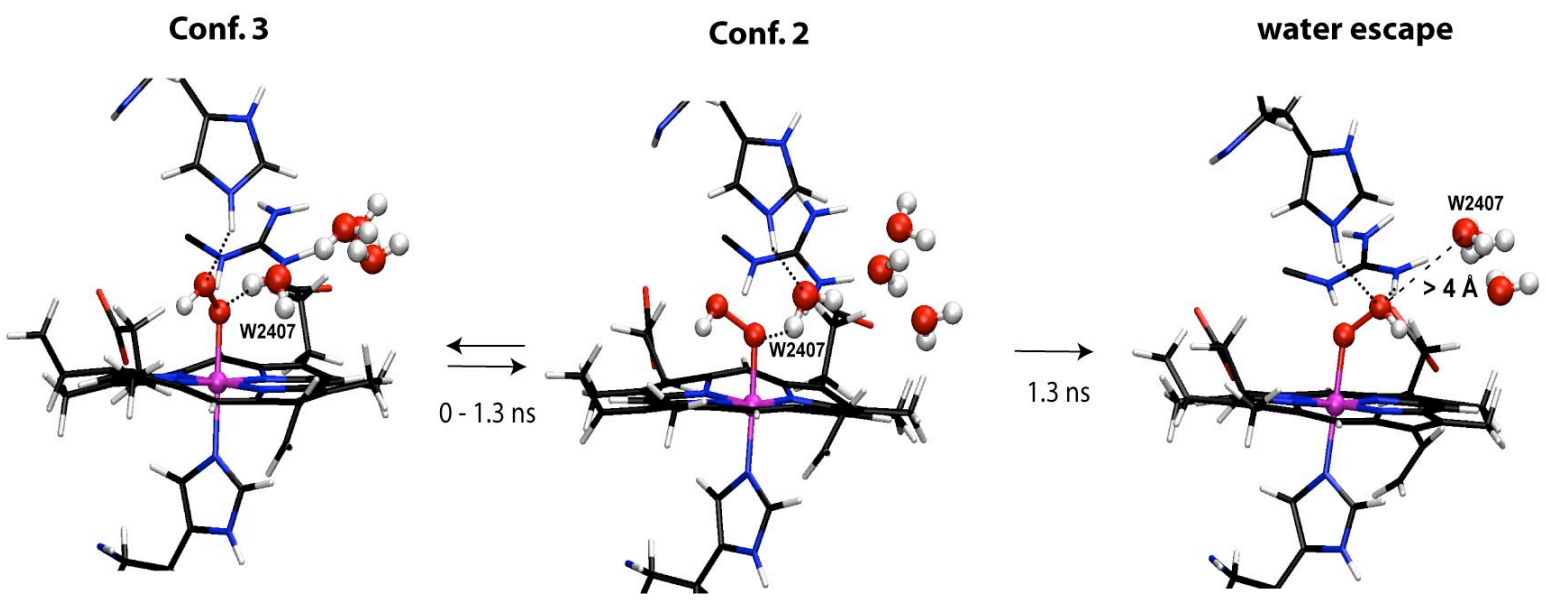

Figure S9. Representative snapshots of the classical MD simulation of Cpd 0. During the first nanosecond, the results are the same as in the CPMD simulation (i.e. frequent interconversion

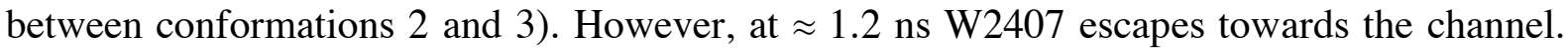
At the same time, the $\mathrm{OOH}$ ligand rotates around the $\mathrm{Fe}-\mathrm{O}_{1}$ bond and a hydrogen bond between $\mathrm{O}_{2}$ and the side chain of Arg38 is formed. The resulting conformation of the peroxide relative to His42 and Arg38 is identical to the one previously previously found in the absence of W2407 (E. Derat \& S. Shaik, J. Phys. Chem. B, 2006, 110, 10526-10533), thus it is perfectly set up to receive a proton from $\mathrm{His}_{-} \mathrm{H}^{+} 42$ and undergo heterolytic $\mathrm{O}-\mathrm{O}$ cleavage. 


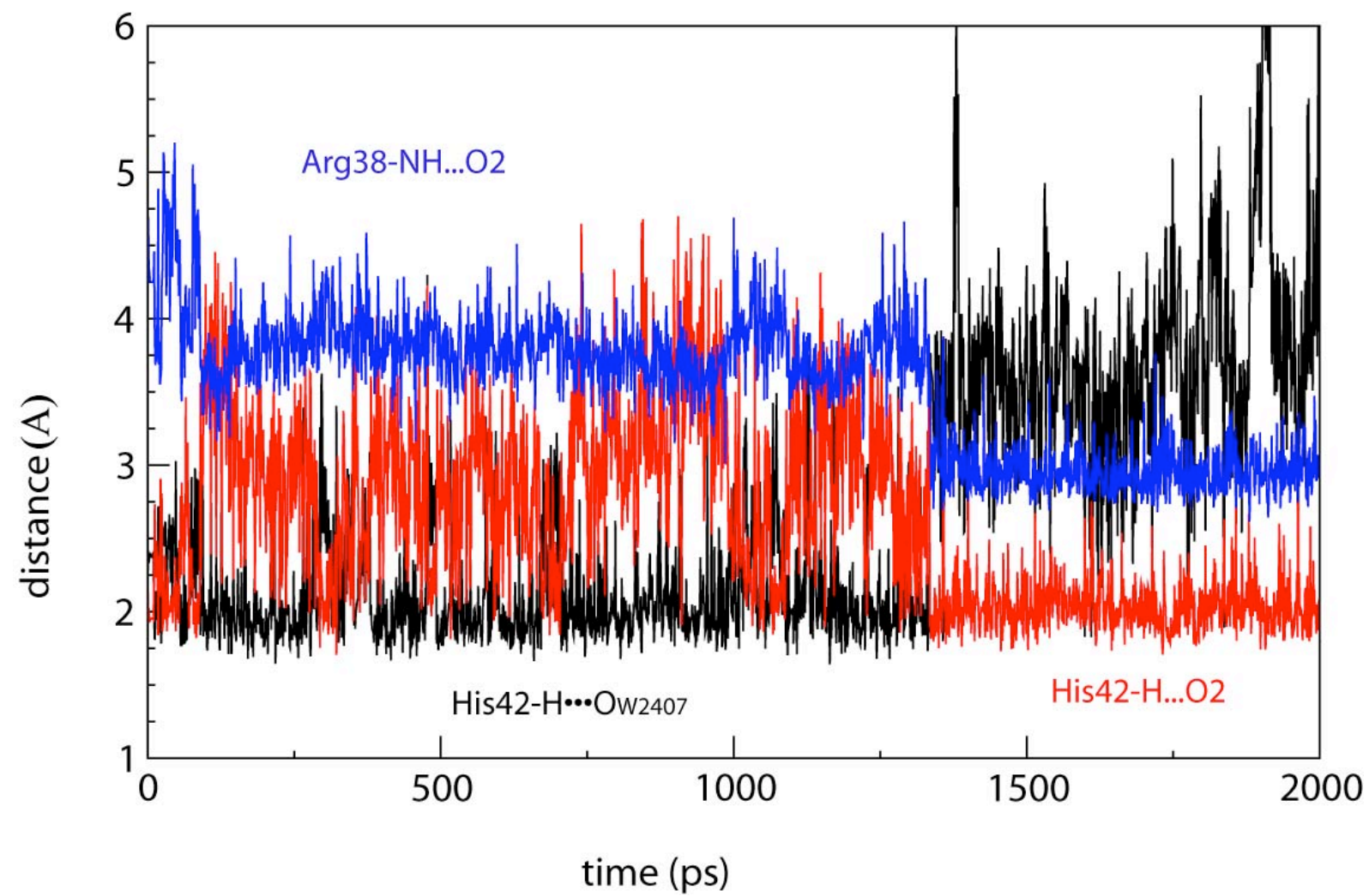

Figure S10 : Time evolution of the His42- $\mathrm{H}^{\cdots} \mathrm{O}_{\mathrm{w} 2407}$ (black line), His42- $\mathrm{H}^{\cdots} \mathrm{O}_{2}$ (red line) and Arg38- $\mathrm{NH} \cdots \mathrm{O}_{2}$ distances during the classical MD simulation. Escape of W2407 occurs at $\approx 1300 \mathrm{ps}$ and it is characterized by : (a) an increase of His42- $\mathrm{H} \cdots \mathrm{O}_{\mathrm{w} 2407}$ (i.e. water escape); (b) a decrease of His42- $\mathrm{H}^{\cdots} \mathrm{O}_{2}$; (c) a decrease of $\operatorname{Arg} 38-\mathrm{NH} \cdots \mathrm{O}_{2}$ (i.e. formation of a new Hbond between Arg38 and the distal oxygen of the peroxide). 